\title{
ALGEBRAIC GEOMETRIC INTERPRETATION OF DONALDSON'S POLYNOMIAL INVARIANTS
}

\author{
JUN LI
}

\section{Introduction}

The purpose of this paper is to understand Donaldson's polynomial invariants of four-manifolds in the context of algebraic geometry. In particular, we explore, among other things, the possibility of defining Donaldson's polynomial invariants of algebraic surfaces by relying on the intersection theory of algebraic varieties.

Let $X$ be any smooth algebraic surface and let $H$ be a very ample line bundle on $X$ with $\mathbf{g}$ its Hodge metric. For any $d>0$, there is a unique $\mathrm{SU}(2)$ bundle $E$ over $X$ of second Chern class $d$. We denote by $\mathscr{N}_{d}(\mathrm{~g})$ the space of gauge equivalent classes of irreducible anti-self-dual (ASD) (with respect to g) $\mathrm{SU}(2)$ connections on $E$. According to Uhlenbeck's weak compactness theorem, there is a canonical compactification of $\mathscr{N}_{d}(\mathbf{g})$ [2]. Let $\overline{\mathscr{N}}_{d}(\mathbf{g})$ be such a compactification. On the other hand, any irreducible ASD connection on $E$ induces a holomorphic structure on $E$, which turns out to be $\mu$-stable with respect to the divisor $H$ [1]. Thus, $\mathscr{N}_{d}(\mathbf{g})$ can be identified with a subset of the moduli scheme $\mathscr{M}_{d}(H)$ of rank two $H$-semistable sheaves $F$ with $\operatorname{det} F=\mathscr{O}$ and $c_{2}(F)=d$. $\mathscr{M}_{d}(H)$ is projective, thus is compact [8]. In this sense, $\mathscr{M}_{d}(H)$ is another compactification of the space $\mathscr{N}_{d}(\mathbf{g})$. It is both interesting and important to understand the relation between Uhlenbeck's compactification $\overline{\mathscr{N}}_{d}(\mathbf{g})$ and Gieseker's compactification $\mathscr{M}_{d}(H)$. Based on Uhlenbeck's compactification $\overline{\mathscr{N}}_{d}(\mathbf{g})$, Donaldson introduced a series of polynomials $\left\{q_{k}\right\}$ of the four-manifold $X$. The polynomials are defined by calculating the selfintersection numbers of proper subsets of $\overline{\mathscr{N}}_{d}(\mathbf{g})$ when $\mathbf{g}$ is a generic Riemannian metric. In the case where the manifold $X$ is simply connected and $b_{+}^{2}(X)$ is odd and strictly larger than 1 , he showed that these numbers are well defined and are indeed invariants of the smooth structure of the four-manifold $X$ [3].

Received December 16, 1991. 
The first main theorem of the paper is

Theorem 0.1. There is a complex structure on $\bar{N}_{d}(\mathbf{g})$ making it a reduced projective scheme. Further, if we let $\mathscr{M}_{d}^{u}(H)$ be the open subset of $\mathscr{M}_{d}(H)$ consisting of locally free $\mu$-stable sheaves and let $\overline{\mathscr{M}}_{d}^{\mu}(H)$ be the closure of $\mathscr{M}_{d}^{\mu}(H)$ endowed with the reduced scheme structure, then there is a morphism

$$
\gamma: \overline{\mathscr{M}}_{d}^{\mu}(H) \rightarrow \overline{\mathscr{N}}_{d}
$$

extending the map between the set of $\mu$-stable rank-two vector bundles and the set of gauge equivalent classes of irreducible ASD connections with fixed Chern classes.

The idea of the proof is straightforward. We construct a line bundle $\mathscr{L}_{\mathscr{L}, k}$ on $\mathscr{M}_{d}(H)$ that is the dual of the determinant line bundle of a perfect complex on $\mathscr{M}_{d}(H)$. We show that for large $m$, the space $H^{0}\left(\mathscr{K}_{d}, \mathscr{L}_{\mathscr{M}, k}^{\otimes m}\right)$ is base point free. Then a suitable choice of a subspace $\mathscr{V}_{k}(m) \subset H^{0}\left(\mathscr{M}_{d}(H), \mathscr{L}_{\mathscr{M}, k}^{\otimes m}\right)$ gives rise to a morphism

$$
\mathscr{M}_{d}(H) \rightarrow \mathbf{P}\left(\mathscr{V}_{k}(m)^{\vee}\right) \text {. }
$$

We will show that the image scheme of $\overline{\mathscr{M}}_{d}^{\mu}(H) \subset \mathscr{M}_{d}(H)$ under this map is homeomorphic to Uhlenbeck's compactification $\overline{\mathscr{N}}_{d}(\mathbf{g})$.

By using what we know about the relation between $\mathscr{M}_{d}(H)$ and $\overline{\mathscr{N}}_{d}(\mathbf{g})$, we can redefine part of Donaldson's polynomial invariants of the algebraic surface in the algebraic geometric context. More precisely, to any sufficiently large integer $d$ and ample divisor $H$ which is $d$-generic in the sense that whenever $D \in \mathrm{NS}(X)$ with $D \cdot D \geq-d$, then $H \cdot D \neq 0$, we then construct a homomorphism $\rho: \mathrm{NS}(X) \rightarrow \mathrm{NS}\left(\mathscr{M}_{d}\right)$ and define the polynomials $\delta_{d}(H)$ by

$$
\delta_{d}(H)\left(\alpha_{1}, \cdots, \alpha_{k}\right)=\left[\rho\left(\alpha_{1}\right) \cdots \rho\left(\alpha_{k}\right)\right]\left(\mathscr{M}_{d}\right),
$$

where $\alpha_{1}, \cdots, \alpha_{k} \in H^{1,1}(X, \mathbb{Z})$ and $k=\operatorname{dim} \mathscr{M}_{d}(H)$. We then show

Theorem 0.2. Let $X$ be a smooth simply connected algebraic surface with $p_{g} \geq 1$, let $\mathrm{NS}(X)_{\mathbb{Q}}^{+} \subset \mathrm{NS}(X) \otimes_{\mathbb{Z}} \mathbb{Q}$ be the cone spanned by ample $\mathbb{Q}$-divisors, and assume $\mathscr{C} \subset \mathrm{NS}(X)_{\mathbb{Q}}^{+}$is any compact subcone. Then there is a constant $A(\mathscr{C})$ such that whenever $d \geq A(\mathscr{C})$ and $H \in \mathscr{C}$ is $d$-generic, then the polynomial $\delta_{d}(H): \operatorname{Symm}^{k} H^{1,1}(X, \mathbb{Z}) \rightarrow \mathbb{Z}$ with $k=\operatorname{dim} \mathscr{M}_{d}(H)$ is identical to the restriction to $\operatorname{Symm}^{k} H^{1,1}(X, \mathbb{Z}) \subset$ $\operatorname{Symm}^{k} H^{2}(X, \mathbb{Z})$ of Donaldson's polynomial invariant $q_{k}$.

The paper is organized as follows. In $\S 1$, we recall the construction of the determinant line bundle and study the descent problem of the moduli 
functor associated to $\mathscr{M}_{d}(H)$. In $\S 2$, we deal with the space $\mathscr{V}_{k}(m) \subset$ $H^{0}\left(\mathscr{M}_{d}, \mathscr{L}_{\mathscr{M}, k}^{\otimes m}\right)$ and analyze the image scheme $\mathscr{M}_{d} \rightarrow \mathbf{P}\left(\mathscr{V}_{k}(m)^{\vee}\right)$ in $\S 3$. The proof of Theorem 0.1 occupies $\S 4$. Finally, in $\S 5$ we compare the polynomials $\delta_{k}(H)$ with $q_{k}$ and prove Theorem 0.2 .

\section{Determinant line bundles on $\mathscr{M}_{d}$}

Let $X$ be a smooth algebraic surface over the complex number field $k=\mathbb{C}$ and let $H$ be an ample line bundle on $X$. For any sheaf $F$ on $X$, we denote the Hilbert polynomial of $F$ by

$$
\chi_{F}(n)=\sum(-1)^{i} h^{i}\left(F \otimes H^{\otimes n}\right)
$$

A sheaf $F$ on $X$ is said to be stable (resp. semistable) with respect to $H$ if $F$ is coherent, torsion free, and, for any proper subsheaf $L \subset F$,

$$
\frac{1}{\operatorname{rank}(L)} \chi_{L}(n) \prec \frac{1}{\operatorname{rank}(F)} \chi_{F}(n) \quad(\text { resp. } \preceq) \text {. }
$$

Here for polynomials $p$ and $q$ with real coefficients, we say $p \prec q$ (resp. $p \preceq q$ ) if for $n \gg 0, p(n)<q(n)$ (resp. $p(n) \leq q(n)$ ). In this paper, unless otherwise stated, we will fix the polarization $H$ and study the space of sheaves that are semistable with respect to $H$.

In [8], Gieseker showed that there is a scheme coarsely representing the moduli functor of semistable sheaves on $X$ with fixed Hilbert polynomial. More precisely, if we let $\mathscr{S}$ be the set of schemes separable over $\mathbb{C}$ and let $\mathscr{E}^{d}(H)$ be the set of all rank two semistable sheaves $F$ on $X$ with $\operatorname{det} F=\mathscr{O}$ and $c_{2}(F)=d$, then we can define a functor $\mathscr{F}_{H}^{d}: \mathscr{S} \rightarrow \mathscr{E}^{d}(H)$ as follows. For any $S \in \mathscr{S}, \mathscr{F}_{H}^{d}(S)$ is the set of all families of sheaves $E_{S}$ in $\mathscr{E}^{d}(H)$ on $X \times S$ flat over $S$ such that $\operatorname{det} E_{S}=p_{2}^{*} L$, where $L$ is a line bundle on $S$. We identify two families $F_{1}$ and $F_{2}$ on $X_{S}$ if $F_{1}=F_{2} \otimes p_{2}^{*} L^{\prime}$, where $L^{\prime}$ is a line bundle on $S$ and $p_{2}$ (resp. $p_{1}$ ) is the projection onto the second (resp. first) factor. We have the following theorem that follows directly from Gieseker's proof.

Theorem 1 (Gieseker [8]). There is a projective scheme $\mathscr{M}_{d}(H)$ coarsely representing the functor $\mathscr{F}_{H}^{d}$.

We remark that $\mathscr{M}_{d}(H)$ does depend on the choice of $H$. In the sequel, we abbreviate $\mathscr{M}_{d}(H)$ to $\mathscr{M}_{d}$ when the polarization $H$ is clear from the context.

The goal of this section is to introduce a family of determinate line bundles on $\mathscr{M}_{d}$. First let us recall the construction and basic properties of the determinant line bundle of a perfect complex on a quasi-projective 
scheme. Assume that $Z$ is a quasi-projective scheme. A complex of sheaves $\mathscr{F}^{*}$ on $Z$ is said to be a perfect complex if locally on $Z$ there exists a bounded complex $\mathscr{Y}_{U}^{\dot{0}}$ of finite locally free $\mathscr{O}_{U}$-modules that is quasi-isomorphic to $\mathscr{F}_{\mid U}^{*}$, where $U \subset Z$. It was shown in [12] that there is a determinant functor from the category of perfect complexes on $Z$ to the category of graded invertible sheaves on $Z$. Indeed, let $\mathscr{F}^{\circ}$ be any perfect complex and let

$$
\mathscr{y}^{0} \rightarrow \mathscr{y}^{1} \rightarrow \cdots \rightarrow y^{r}
$$

be a finite locally free $\mathscr{O}_{Z}$-module that is quasi-isomorphic to $\mathscr{F}^{\cdot}$. Then

$$
\operatorname{det}\left(\mathscr{F}^{\cdot}\right)=\bigotimes_{i=0}^{r}\left(\bigvee^{\text {top }} \mathscr{Y}^{i}\right)^{(-1)^{i}}
$$

Let $Y$ and $Z$ be quasi-projective schemes and let $p: Y \rightarrow Z$ be a proper morphism that is flat over $Z$. It is known that if $\mathscr{F}^{\cdot}$ is a perfect complex on $Y$, then $R^{*} p_{*} \mathscr{F}^{*}$ is again a perfect complex. Hence to every perfect complex on $Y$ we associate an invertible sheaf $\operatorname{det}\left(R^{*} p_{*} \mathscr{F}^{*}\right)$ on $Z$. In the following, we consider the case where $p: Y \rightarrow Z$ is a flat family of reduced curves. Assume that $F$ is a locally free sheaf on $Y$, and that $D \subset Y$ is a divisor which is a multiple section of $p$ such that $\mathscr{O}(D)$ is ample relative to $p$. Then, since $Z$ is quasi-projective, there is a large $n$ so that $R^{1} p_{*}(F(n D))=0$. Since $F$ is locally free, we have the exact sequence

$$
0 \rightarrow F \rightarrow F(n D) \rightarrow F_{\mid n D} \rightarrow 0 .
$$

The corresponding long exact sequence

$$
0 \rightarrow p_{*} F \rightarrow p_{*} F(n D) \stackrel{\sigma}{\longrightarrow} p_{*} F_{\mid n D} \rightarrow R^{1} p_{*} F \rightarrow 0
$$

on $Z$ states that the complex $p_{*} F \stackrel{\times 0}{\rightarrow} R^{1} p_{*} F$ is quasi-isomorphic to the complex $R^{0} p_{*} F(n D) \stackrel{\sigma}{\rightarrow} p_{*} R_{\mid n D}$. Note that both $R^{0} p_{*} F(n D)$ and $p_{*} F_{\mid n D}$ are locally free. Thus

$$
\operatorname{det}\left(R^{\circ} p_{*} F\right)=\operatorname{det}\left(R^{0} p_{*} F(n D)\right) \otimes \operatorname{det}\left(p_{*}\left(F_{\mid n D}\right)\right)^{-1} .
$$

In general, if $\mathscr{F}^{*}=\left\{F^{*}\right\}$ is a complex of finite locally free sheaves on $Y$ of the form (1.1), then

$$
\operatorname{det}\left(R^{\cdot} p_{*} \mathscr{F}^{\cdot}\right)=\bigotimes_{i=0}^{r} \operatorname{det}\left(R^{\cdot} p_{*} F^{i}\right)^{(-1)^{i}}
$$


We remark that such a functor $\operatorname{det}\left(R^{\cdot} p_{*}(\cdot)\right)$ is canonical under the base change and that if

$$
0 \rightarrow \mathscr{F}_{1}^{\cdot} \rightarrow \mathscr{F}_{2}^{\cdot} \rightarrow \mathscr{F}_{3}^{\cdot} \rightarrow 0
$$

is a triangle of perfect complexes, then

$$
\operatorname{det}\left(R^{\circ} p_{*} \mathscr{F}_{1}^{*}\right) \otimes \operatorname{det}\left(R^{\circ} p_{*} \mathscr{F}_{3}^{*}\right)=\operatorname{det}\left(R^{\circ} p_{*} \mathscr{F}_{2}^{*}\right) .
$$

We refer to [12] for the details of the proof.

Let $S$ be a quasi-projective scheme. Then two line bundles $A_{1}$ and $A_{2}$ on $S$ are said to be algebraic equivalent if there is a smooth irreducible variety $T$, a line bundle $A_{T}$ on $S \times T$, and two closed points $t_{1}, t_{2} \in T$ such that $A_{1}$ (resp. $A_{2}$ ) is isomorphic to the restriction of $A_{T}$ to $S \times\left\{t_{1}\right\}$ (resp. $S \times\left\{t_{2}\right\}$ ). We denote by $\mathrm{NS}(S)$ the quotient of $\operatorname{Pic}(S)$ by the algebraic equivalence relation. Now let $X$ be a smooth algebraic surface and let $F_{S}$ be a family of torsion free sheaves on $X \times S$ flat over $S$. We define a homomorphism

$$
\rho_{F}: \mathrm{NS}(X) \rightarrow \mathrm{NS}(S)
$$

as follows. Since $X$ is smooth, $\operatorname{dim} X=2$ and $F_{S}$ is flat over $S, F_{S}$ belongs to the exact sequence

$$
0 \rightarrow Q_{2} \rightarrow Q_{1} \rightarrow F_{S} \rightarrow 0,
$$

where $Q_{1}$ and $Q_{2}$ are locally free sheaves on $X \times S$. For any smooth divisor $C \subset X$, we tensor (1.9) by $\mathscr{O}_{C \times S}$,

$$
\begin{aligned}
0 \rightarrow \operatorname{Tor}_{1}\left(F_{S}, \mathscr{O}_{C \times S}\right) & \rightarrow Q_{2} \otimes_{\mathscr{O}_{X \times S}} \mathscr{O}_{C \times S} \\
& \rightarrow Q_{1} \otimes_{\mathscr{O}_{X \times S}} \mathscr{O}_{C \times S} \rightarrow F_{S} \otimes_{X \times S} \mathscr{Q}_{C \times S} \rightarrow 0 .
\end{aligned}
$$

We claim that $\operatorname{Tor}_{1}\left(F_{S}, \mathscr{O}_{C \times S}\right)=0$. Indeed, the set $\operatorname{Sing}\left(F_{S}\right) \subset X \times S$ consisting of closed points, where $F_{S}$ ceases to be locally free, is a closed subset of codimension at least two. Clearly, $\operatorname{Supp}\left(\operatorname{Tor}_{1}\left(F_{S}, \mathscr{O}_{C \times S}\right)\right) \subset$ $\operatorname{Sing}\left(F_{S}\right)$. Thus,

$$
\operatorname{Tor}_{1}\left(F_{S}, \mathscr{O}_{C \times S}\right) \subset Q_{2} \otimes_{\mathscr{O}_{X \times S}} \mathscr{O}_{C \times S},
$$

as a torsion subsheaf of $Q_{2} \otimes_{\mathscr{O}_{X \times S}} \mathscr{O}_{C \times S}$, is a trivial subsheaf since the later is locally free on $C \times S$. Hence, $\operatorname{Tor}_{1}\left(F_{S}, \mathscr{O}_{C \times S}\right)=0$. We acknowledge that in the sequel we will use $F_{S \mid C}$ to denote the restriction of $F_{S}$ on $X \times S$ to $C \times S$. In particular, $F_{S \mid C}$ is a prefect complex on $C \times S$. Now by using the smooth morphism $p_{2}: C \times S \rightarrow S$, we can form the determinant line bundle of the perfect complex:

$$
R^{\circ} p_{2, *}\left(F_{S \mid C} \otimes p_{1}^{*} \theta_{C}\right),
$$


where $\theta_{C}$ is a line bundle on $C$ with $\operatorname{deg} \theta_{C}=g(C)-1$. We denote such a line bundle by

$$
\operatorname{Det}\left(R p_{2, *}\left(F_{S \mid C} \otimes p_{1}^{*} \theta_{C}\right)\right)=\operatorname{det}\left(R^{*} p_{2, *}\left(F_{S \mid C} \otimes p_{1}^{*} \theta_{C}\right)\right),
$$

whose inverse is denoted by $\mathscr{L}_{F}\left(C, \theta_{C}\right)$, which may depend on the choice of $\theta_{C} \in \mathrm{Jac}^{g(C)-1}(C)$. Since $\mathrm{Jac}^{g(C)-1}(C)$ is connected and smooth, it follows that $\mathscr{L}_{F}\left(C, \theta_{C_{1}}\right) \sim_{\text {alg }} \mathscr{L}_{F}\left(C, \theta_{2}\right)$ for $\theta_{C_{1}}$ and $\theta_{C_{2}}$ in $\mathrm{Jac}^{g(C)-1}(C)$. So if we denote by $\left[\mathscr{L}_{F}\left(C, \theta_{C}\right)\right]$ the algebraic equivalence class of $\mathscr{L}_{F}\left(C, \theta_{C}\right)$ in $\mathrm{NS}(S)$, then $\left[\mathscr{L}_{F}\left(C, \theta_{C}\right)\right]$ is independent on the choice of $\theta_{C}$. Finally, an arbitrary divisor $C$ can be written as the difference of two ample divisors: $C=C_{1}-C_{2}$. Then we define $\rho(C)=$ $\left[\mathscr{L}_{F}\left(C_{1}, \theta_{C}\right)\right]-\left[\mathscr{L}_{F}\left(C_{2}, \theta_{C_{2}}\right)\right]$. To show that $\rho_{F}(C)=\left[\mathscr{L}_{F}\left(C_{1}, \theta_{C_{1}}\right)\right]-$ $\left[\mathscr{L}_{F}\left(C_{2}, \theta_{C_{2}}\right)\right]$ is a well-defined homomorphism from $\mathrm{NS}(X)$ to $\mathrm{NS}(S)$, we need to show that $\left[\mathscr{L}_{F}\left(C_{1}, \theta_{C_{1}}\right)\right]=\left[\mathscr{L}_{F}\left(C_{2}, \theta_{C_{2}}\right)\right]$ if $C_{1} \sim_{\text {alg }} C_{2}$, and that $\left[\mathscr{L}_{F}\left(C_{0}, \theta_{C_{0}}\right)\right]=\left[\mathscr{L}_{F}\left(C_{1}, \theta_{C_{1}}\right)\right]+\left[\mathscr{L}_{F}\left(C_{2}, \theta_{C_{2}}\right)\right]$ if $C_{0} \sim_{\text {alg }} C_{1}+C_{2}$. We remark that by $\theta_{C}$ we always mean a line bundle on $C$ with $\operatorname{deg} \theta_{C}=$ $g(C)-1$.

Lemma 1.1. With the notation as above, assume that $C_{1}$ and $C_{2}$ are two smooth algebraic equivalent divisors of $X$ and that $\theta_{C_{i}} \in \mathrm{Jac}^{g\left(C_{i}\right)-1}\left(C_{i}\right)$, $i=1,2$. Then $\left[\mathscr{L}_{F}\left(C_{1}, \theta_{C_{1}}\right)\right]=\left[\mathscr{L}_{F}\left(C_{2}, \theta_{C_{2}}\right)\right]$.

Lemma 1.2. With the notation as above, assume the following: $C_{0}, C_{1}$, and $C_{2}$ are smooth divisors with $C_{0}$ linear equivalent to $C_{1}+C_{2}, C_{1}$ and $C_{2}$ meet transversally, $C_{0}$ is very ample, and $C_{0} \cap C_{1} \cap C_{2}=\varnothing$. Then $\left[\mathscr{L}_{F}\left(C_{0}, \theta_{C_{0}}\right)\right]=\left[\mathscr{L}_{F}\left(C_{1}, \theta_{C_{1}}\right)\right]+\left[\mathscr{L}_{F}\left(C_{2}, \theta_{C_{2}}\right)\right]$.

Proof. We will only prove Lemma 1.2. Lemma 1.1 can be proved similarly. Without loss of generality, we can assume that $F_{S}$ is locally free and that $C_{0}$ is generic. Let $D_{t}$ be a linear series of divisors on $X$ so that $D_{\alpha}=C_{0}$ and $D_{\beta}=C_{1}+C_{2}$. By blowing up the surface $X$ along the base points of the linear series, we obtain a smooth surface $Y$ and a projection $q: Y \rightarrow \mathbf{P}^{1}$ such that $q^{-1}(c)$ is a divisor in the linear series for any $c \in \mathbf{P}^{1}$. Since $C_{0}$ is very ample, $C_{0} \cap C_{1}$ and $C_{0} \cap C_{2}$ are nonempty. Let $z_{1} \in C_{0} \cap C_{1}$ and $z_{2} \in C_{0} \cap C_{2}$ be closed points, and let $E_{1}$ and $E_{2}$ be exceptional divisors in $Y$ over $z_{1}$ and $z_{2}$, respectively. Then we can choose appropriate integers $a$ and $b$ so that the line bundle $\theta=\mathscr{O}\left(a E_{1}+b E_{2}\right)$ on $Y$ has the property that $\operatorname{deg}\left(\theta, C_{0}\right)=g\left(C_{0}\right)-1$ and $\operatorname{deg}\left(\theta, C_{2}\right)=g\left(C_{2}\right)-1$. Let $p: Y \rightarrow X$ be the projection, let $f=$ $(q$, id $): Y \times S \rightarrow \mathbf{P}^{1} \times S$ and $\tilde{p}=(p$, id $): Y \times S \rightarrow X \times S$, and let $p_{P}: Y \times S \rightarrow \mathbf{P}^{1}$. Consider the locally free sheaf $\mathscr{F}=\tilde{p}^{*} F_{S}$ on $Y \times S$ 
and define the line bundle

$$
\mathscr{L}_{F}=\operatorname{Det}\left(R f_{*}\left(\tilde{p}^{*} F_{S} \otimes p_{1}^{*} \theta\right)\right)^{-1}
$$

on $\mathbf{P}^{1} \times S$. The lemma will be proved if we can show that

$$
\left.\mathscr{L}_{F}\right|_{p_{P}^{-1}(\alpha)}=\mathscr{L}_{F}\left(C_{0}, \theta_{\mid C_{0}}\right),
$$

$$
\begin{aligned}
& \left.\mathscr{L}_{F}\right|_{p_{P}^{-1}(\beta)} \\
& \quad=\operatorname{Det}\left(R p_{2, *} F_{S \mid C_{1}} \otimes p_{1}^{*} \theta_{C_{1}}\right)^{-1} \otimes \operatorname{Det}\left(R p_{2, *} F_{S \mid C_{2}} \otimes p_{1}^{*} \theta_{C_{2}}\right)^{-1}
\end{aligned}
$$

where $\theta_{C_{1}}$ and $\theta_{C_{2}}$ are line bundles on $C_{1}$ and $C_{2}$ defined by $\theta_{C_{1}}=$ $\theta\left(-C_{2}\right)_{\mid C_{1}}$ and $\theta_{C_{2}}=\theta_{\mid C_{2}}$, respectively. Note that $\operatorname{deg}\left(\theta\left(-C_{2}\right), C_{1}\right)=$ $g\left(C_{1}\right)-1$. (1.12) follows from the base change property of the determinant line bundle. (1.13) can be seen as follows. By the base change property, $\left.\mathscr{L}_{F}\right|_{p_{P}^{-1}(\beta)}$ is isomorphic to the dual of the determinant line bundle of the complex $R^{*} p_{2, *}\left(F_{S \mid C_{1} \cup C_{2}} \otimes p_{1}^{*} \theta\right)$. Since $F_{S}$ is locally free, we have the exact sequence

$$
0 \rightarrow F_{S \mid C_{1}} \otimes p_{1}^{*} \theta_{\mid C_{1}}\left(-C_{2}\right) \rightarrow F_{S \mid C_{1} \cup C_{2}} \otimes p_{1}^{*} \theta_{\mid C_{1} \cup C_{2}} \rightarrow F_{S \mid C_{2}} \otimes p_{1}^{*} \theta_{\mid C_{2}} \rightarrow 0,
$$

and therefore the triangle of complexes

$$
\begin{aligned}
0 & \rightarrow R p_{2, *}\left(F_{S \mid C_{1}} \otimes p_{1}^{*} \theta_{C_{1}}\right) \rightarrow R p_{2, *}\left(F_{S \mid C_{1} \cup C_{2}} \otimes p_{1}^{*} \theta_{\mid C_{1} \cup C_{2}}\right) \\
& \rightarrow R p_{2, *}\left(F_{S \mid C_{2}} \otimes p_{1}^{*} \theta_{C_{2}}\right) \rightarrow 0 .
\end{aligned}
$$

Hence,

$$
\begin{aligned}
\left.\mathscr{L}_{F}\right|_{p_{P}^{-1}(\beta)} & =\operatorname{det}\left(R^{*} p_{2, *}\left(F_{S \mid C_{1} \cup C_{2}} \otimes p_{1}^{*} \theta_{\mid C_{1} \cup C_{2}}\right)\right)^{-1} \\
& =\operatorname{det}\left(R^{\cdot} p_{2, *}\left(F_{S \mid C_{1}} \otimes p_{1}^{*} \theta_{C_{1}}\right)\right)^{-1} \otimes \operatorname{det}\left(R_{p_{2, *}}\left(F_{S \mid C_{2}} \otimes p_{1}^{*} \theta_{C_{2}}\right)\right)^{-1}
\end{aligned}
$$

which proves (1.13). So, $\left[\mathscr{L}_{F}\left(C_{1} \cup C_{2}\right)\right]=\left[\mathscr{L}_{F}\left(C_{1}\right)\right]+\left[\mathscr{L}_{F}\left(C_{2}\right)\right]$. q.e.d.

Applying Lemmas 1.1 and 1.2, we arrive at

Proposition 1.3. Let $S$ be any quasi-projective scheme and let $F_{S}$ be a family of coherent, torsion free sheaves on $X \times S$ flat over $S$. Then there is a homomorphism $\rho: \mathrm{NS}(X) \rightarrow \mathrm{NS}(S)$. For any smooth divisor $C \in \mathrm{NS}(X), \rho(C)$ is the algebraic equivalence class in $\mathrm{NS}(S)$ represented by the line bundle

$$
\mathscr{L}_{F}\left(C, \theta_{C}\right)=\operatorname{Det}\left(R p_{2, *} F_{S \mid C} \otimes p_{1}^{*} \theta_{C}\right)^{-1},
$$

where $\theta_{C}$ is a line bundle on $C$ with $\operatorname{deg}\left(\theta_{C}\right)=g(C)-1$. Further, the homomorphism $\rho$ is canonical in the sense that if $S^{\prime}$ is another scheme, 
$g: S^{\prime} \rightarrow S$, and $F_{S^{\prime}}=g^{*} F_{S}$ is the family of torsion free sheaves on $X \times S^{\prime}$, then the homomorphism $\rho^{\prime}: \mathrm{NS}(X) \rightarrow \mathrm{NS}\left(S^{\prime}\right)$ is identical to $g^{*} \circ \rho$.

In case $F_{S}$ is a flat family of torsion sheaves with $\operatorname{det} F_{S}=p_{2}^{*} L$ for some line bundle $L$ on $S$, we have the following lemma which reveals the significance of choosing the line bundle $\theta_{C}$ which satisfies $\chi\left(\theta_{C}\right)=0$.

Lemma 1.4. Let $F_{1}$ and $F_{2}$ be two families of torsion free sheaves on $X \times S$ flat over $S$ such that $\operatorname{det} F_{1}=p_{2}^{*} L$. Assume $F_{1}$ and $F_{2}$ are equivalent in the sense that there is a line bundle $P$ on $S$ such that $F_{1} \cong$ $F_{2} \otimes p_{2}^{*} P$. Then, for any smooth curve $C \subset X$,

$$
\operatorname{Det}\left(R p_{2, *} F_{1 \mid C} \otimes p_{1}^{*} \theta_{C}\right) \cong \operatorname{Det}\left(R p_{2, *} F_{2 \mid C} \otimes p_{1}^{*} \theta_{C}\right) \text {. }
$$

Proof. We have

$$
\begin{aligned}
\operatorname{Det}\left(R p_{2, *} F_{1 \mid C} \otimes p_{1}^{*} \theta_{C}\right) & =\operatorname{Det}\left(R p_{2, *}\left(F_{2 \mid C} \otimes p_{2}^{*} P_{\mid C} \otimes p_{1}^{*} \theta_{C}\right)\right) \\
& =\operatorname{Det}\left(R p_{2, *} F_{2 \mid C} \otimes p_{1}^{*} \theta_{C}\right) \otimes P^{\otimes \chi\left(F_{2, s \mid C} \otimes p_{1}^{*} \theta_{C}\right)} \\
& =\operatorname{Det}\left(R p_{2, *} F_{2 \mid C} \otimes p_{1}^{*} \theta_{C}\right) .
\end{aligned}
$$

The last equality holds because $\chi\left(F_{2, s \mid C} \otimes p_{1}^{*} \theta_{C}\right)=0$. Here we have used the fact that $\operatorname{det} F_{2, s}=\mathscr{O}$ for all closed points $s \in S$. q.e.d.

In the remainder of this section, we will study the existence of the homomorphism $\rho: \mathrm{NS}(X) \rightarrow \mathrm{NS}\left(\mathscr{M}_{d}\right)$. To apply Proposition 1.3 directly, we need a universal sheaf on $X \times \mathscr{M}_{d}$. By a universal sheaf of $\mathscr{M}_{d}$ we mean a sheaf $F$ on $X \times \mathscr{M}_{d}$ such that, for any point $s \in \mathscr{M}_{d}$, the sheaf $F_{s}$ which is the restriction of $F_{S}$ to $X \times\{s\}$ is isomorphic to the sheaf represented by the point $s \in \mathscr{M}_{d}$. When $d$ is odd, such a universal sheaf does exist.

Proposition 1.5. When $d$ is odd, there is a homomorphism

$$
\rho: \mathrm{NS}(X) \rightarrow \mathrm{NS}\left(\mathscr{M}_{d}\right)
$$

such that, for any smooth divisor $C \in \mathrm{NS}(X), \rho(C)$ is the algebraic equivalence class represented by the line bundle $\operatorname{Det}\left(R p_{2, *} F_{\mid C} \otimes p_{1}^{*} \theta_{C}\right)^{-1}$, where $F$ is the universal sheaf on $X \times \mathscr{M}_{d}$.

Proof. By Maruyama [14, Theorem 6.11], the universal sheaf does exist. Since $\mathscr{K}_{d}$ is projective, by applying Proposition 1.3 directly, we get the desired homomorphism. q.e.d.

When $d$ is even, the universal sheaf does not exist even locally near a semistable point. To remedy this difficulty, a discussion of the proof of the existence of moduli scheme $\mathscr{M}_{d}$ is in order (see [8] for details).

Let $\mathscr{E}^{d}(n)$ be the set of all rank-two $H$-semistable sheaves $F$ such that $\operatorname{det} F=H^{\otimes 2 n}$ and $c_{2}\left(F \otimes H^{-n}\right)=d$. There is a constant $A_{d}$ such that 
if $n \geq A_{d}$, then any sheaf $F \in \mathscr{E}^{d}(n)$ has the property that $h^{i}(F)=0$ for $i>0$ and $F$ is generated by the global sections $H^{0}(F)=\mathbb{C}^{N}$. Let Quot $_{\mathscr{Q}^{N}}^{d, 0}$ be the Grothendieck quotient scheme parametrizing all quotient sheaves $F$ of $\bigoplus^{N} \mathscr{O}$ satisfying $\operatorname{det} F(-n)=\mathscr{O}$ and $c_{2}(F(-n))=d$. Clearly any $F \in \mathscr{E}^{d}(n)$ coupled with an identification $H^{0}(F)=\mathbb{C}^{\oplus N}$ is a closed point of Quot $\mathscr{\mathscr { O }}^{d, 0}$, and thus any point in $\mathscr{E}^{d}(n)$ corresponds to a $\operatorname{PGL}(N, \mathbb{C})$ orbit of a closed point of $\operatorname{Quot}_{\mathscr{O}^{N}}^{d, 0}$. Let $\mathscr{Q} \subset \operatorname{Quot}_{\mathscr{Q}^{N}}^{d, 0}$ be the open subset consisting of torsion free good quotient sheaves $\mathscr{O}^{\oplus N} \rightarrow E$ in the sense that the induced homomorphism $H^{0}\left(\mathscr{O}^{N}\right) \rightarrow H^{0}(E)$ is an isomorphism. We set $\mathscr{G}=\operatorname{PGL}(N, \mathbb{C})$. In [8], Gieseker introduced an immersion

$$
\mu: \mathscr{Q} \rightarrow \mathbf{P}\left(\operatorname{Hom}\left(\bigwedge^{2} \mathbb{C}^{N}, H^{0}\left(H^{\otimes 2 n}\right)\right)\right),
$$

where we adopt the convention that $\mathbf{P}\left(\mathbb{C}^{l}\right)$ is the space of all lines in $\mathbb{C}^{l}$. Through the dual action of $\operatorname{GL}(N, \mathbb{C})$ on $\operatorname{Hom}\left(\bigwedge^{2} \mathbb{C}^{N}, H^{0}\left(H^{\otimes 2 n}\right)\right)$, the $\mathrm{GL}(N, \mathbb{C})$-action on the projective space $\mathbf{P}\left(\operatorname{Hom}\left(\bigwedge^{2} \mathbb{C}^{N}, H^{0}\left(H^{\otimes 2 n}\right)\right)\right)$ descends to a $\mathscr{G}$-action and under this $\mathscr{G}$-structure, $\mu$ is a $\mathscr{G}$-morphism. Gieseker showed that $F \in \mathscr{Q}$ is a stable (resp. semistable) quotient sheaf if and only if $\mu(F)$ is a $\mathscr{G}$-stable (resp. $\mathscr{G}$-semistable) point in $\mathbf{P}\left(\operatorname{Hom}\left(\bigwedge^{2} \mathbb{C}^{N}, H^{0}\left(H^{\otimes 2 n}\right)\right)\right)$. Therefore if we let $\mathscr{Q}^{s}$ (resp. $\left.\mathscr{Q}^{s s}\right)$ be the set of $\mathscr{G}$-stable (resp. $\mathscr{G}$-semistable) points in $\mathscr{Q}$, then $\mathscr{M}_{d}^{s}$ is the geometric quotient of $\mathscr{Q}^{s}$ under $\mathscr{G}$ that is isomorphic to a subscheme of $\mathbf{P}\left(\operatorname{Hom}\left(\bigwedge^{2} \mathbb{C}^{N}, H^{0}\left(H^{\otimes 2 n}\right)\right)\right)^{s} / \mathscr{G}$. Its completion $\mathscr{M}_{d}$ is a good quotient of $\mathscr{Q}^{s s}$ by $\mathscr{G}$ which is a closed subscheme of $\mathbf{P}\left(\operatorname{Hom}\left(\bigwedge^{2} \mathbb{C}^{N}\right.\right.$, $\left.\left.H^{0}\left(H^{\otimes 2 n}\right)\right)\right)^{s s} / / \mathscr{G}$.

Let $F_{\mathscr{Q}}$ be the universal quotient sheaf on $X \times \mathscr{Q}$. We denote by $F_{\mathscr{Q}}(-n)$ the sheaf $F_{\mathscr{Q}} \otimes p_{1}^{*} H^{\otimes(-n)}$. Note that $\operatorname{det} F_{\mathscr{Q}}(-n)=p_{2}^{*} I$ for some line bundle $I$ on $\mathscr{Q}$. Let $C \subset X$ be a smooth divisor and let $\theta_{C}$ be a line bundle on $C$ with $\operatorname{deg} \theta_{C}=g(C)-1$. Then,

$$
\mathscr{L}_{\mathscr{Q}}\left(C, \theta_{C}\right)=\operatorname{Det}\left(R p_{2, *} F_{\mathscr{Q}}(-n)_{\mid C} \otimes p_{1}^{*} \theta_{C}\right)^{-1}
$$

is a line bundle on $\mathscr{Q}$. In the following, we shall study when we can descend the line bundle $\mathscr{L}_{\mathscr{Q}}\left(C, \theta_{C}\right)$ to $\mathscr{M}_{d}$. We need the following descent lemma.

Lemma 1.6. Let $E$ be a $\mathscr{G}$-vector bundle on $\mathscr{Q}^{s s}$. Then $E$ can be descended to $\mathscr{M}_{d}$ if and only if for every closed point $s \in \mathscr{Q}^{s s}$ with closed orbit $\mathscr{Q} \cdot s$, the stabilizer $\mathscr{G}_{s} \subset \mathscr{G}$ of $s$ acts trivially on $E_{s}$.

Proof. Note that the condition in Theorem 2.3 of [4] can be relaxed to cover the case where $\mathscr{Y} \rightarrow \mathscr{X}$ is a good quotient by a reductive group 
$\mathscr{G}, \mathscr{Y}$ and $\mathscr{X}$ being any quasi-projective schemes. Since $\mathscr{M}_{d}$ is a good quotient of $\mathscr{Q}^{s s}$, we can invoke this theorem directly to cover our situation.

Proposition 1.7. Let $C \subset X$ be a smooth divisor and $\theta_{C} \in \mathrm{Jac}^{g(C)-1}(C)$. The line bundle $\mathscr{L}_{\mathscr{Q}}\left(C, \theta_{C}\right)$ can be descended to $\mathscr{M}_{d}$ if whenever $F \in \mathscr{Q}^{s s}$ is a split semistable sheaf with splitting $F=J_{1} \oplus J_{2}$, then $c_{1}\left(J_{1}(-n)\right) \cdot C=$ 0 .

Proof. We use the descent lemma to prove this proposition. As before, we denote by $\mathscr{G}$ the group $\operatorname{PGL}(N, \mathbb{C})$. Let

$$
\mathscr{L}_{\mathscr{Q}}\left(C, \theta_{C}\right)=\operatorname{Det}\left(R p_{2, *} F_{\mathscr{Q}}(-n)_{\mid C} \otimes p_{1}^{*} \theta_{C}\right)^{-1}
$$

be the line bundle on $\mathscr{Q}^{s s}$; we first show that $\mathscr{L}_{\mathscr{Q}}\left(C, \theta_{C}\right)$ is a $\mathscr{G}$-bundle. The action of $\mathscr{G}$ on $\mathscr{Q}^{s s}$ does not lift to an action on $F_{\mathscr{Q}}$. To remedy this difficulty, we introduce the general linear group $\operatorname{GL}(N, \mathbb{C})$ acting on $\mathscr{Q}^{s s}$ through surjection $\operatorname{GL}(N, \mathbb{C}) \rightarrow \operatorname{PGL}(N, \mathbb{C})$. There is a canonical lift making $F_{\mathscr{Q}}$ a $\mathrm{GL}(N, \mathbb{C})$-sheaf. The induced action on the determinant line bundle then makes $\mathscr{L}_{\mathscr{Q}}\left(C, \theta_{C}\right)$ a $\mathrm{GL}(N, \mathbb{C})$-bundle. To show that $\mathscr{L}_{\mathscr{Q}}\left(C, \theta_{C}\right)$ is a $\mathscr{G}$-bundle, we need only show that the subgroup $\mathbb{C}^{*} \subset$ $\mathrm{GL}(N, \mathbb{C})$ acts trivially on $\mathscr{L}_{\mathscr{Q}}\left(C, \theta_{C}\right)$.

Let $h=\alpha \cdot$ id: $F_{\mathscr{Q}} \rightarrow F_{\mathscr{Q}}$ be the homothetic where $\alpha \in \mathbb{C}^{*}$. On each irreducible component of $\mathscr{Q}^{s s}$, the induced homomorphism

$$
\begin{aligned}
\operatorname{Det}\left(h_{\mid C}\right): \operatorname{Det} & \left(R p_{2, *} F_{\mathscr{Q}}(-n)_{\mid C} \otimes p_{1}^{*} \theta_{C}\right) \\
& \rightarrow \operatorname{Det}\left(R p_{2, *} F_{\mathscr{Q}}(-n)_{\mid C} \otimes p_{1}^{*} \theta_{C}\right)
\end{aligned}
$$

is a multiplication by the scalar $(\alpha)^{\chi\left(F_{\mathcal{Q}, s}(-n)_{\mid C} \otimes \theta_{C}\right)}$, where $s \in \mathscr{Q}^{s s}$ is any closed point. The exponent is zero since $c_{1}\left(F_{\mathscr{Q}, s}(-n)_{\mid C}\right)=0$. Therefore $\operatorname{Det}\left(h_{\mid C}\right)=$ Id and then $\mathscr{L}_{\mathscr{Q}}\left(C, \theta_{C}\right)$ is a $\mathscr{G}_{\text {-bundle. }}$

For any quotient sheaf $F \in \mathscr{Q}^{s s}, \mathscr{G} \cdot\{F\}$ is closed if and only if either $F$ is stable or $F$ is semistable and splits as a direct sum of two rank-one sheaves, say $F=J_{1} \oplus J_{2}$ [8]. In the former case, the stabilizer of $\{F\}$ is $\{e\}$, while in the second case, $G_{\{F\}}=\mathbb{C}^{*}$ if $J_{1} \neq J_{2}$, and $G_{\{F\}}=$ $\operatorname{PGL}(2, \mathbb{C})$ if $J_{1}=J_{2}$.

By the descent lemma, $\mathscr{L}_{Q}\left(C, \theta_{C}\right)$ descends to a line bundle on $\mathscr{M}_{d}$ if the induced homomorphism (1.16) is an identity homomorphism for any semistable sheaf $F=J_{1} \oplus J_{2}$ and $h \in G_{\{F\}}: J_{1} \oplus J_{2} \rightarrow J_{1} \oplus J_{2}$, the induced homomorphism (1.16) is an identity homomorphism. We check the case where $h=\left(\begin{array}{cc}\alpha & 0 \\ 0 & \alpha^{-1}\end{array}\right)$. An easy argument shows that

$$
\operatorname{Det}\left(h_{\mid C}\right)=(\alpha)^{\chi\left(J_{1}(-n)_{\mid C} \otimes \theta_{C}\right)} \cdot(\alpha)^{-\chi\left(J_{2}(-n)_{\mid C} \otimes \theta_{C}\right)} \cdot \text { Id . }
$$


$\operatorname{Det}\left(h_{\mid C}\right)=$ Id if and only if

$$
\operatorname{deg}\left(J_{1}(-n), C\right)-\operatorname{deg}\left(J_{2}(-n), C\right)=0 .
$$

Since $c_{1}\left(J_{1}(-n)\right)+c_{1}\left(J_{2}(-n)\right)=0,(1.17)$ is equivalent to $c_{1}\left(J_{1}\right) \cdot C=0$. Thus the proposition is established. q.e.d.

When $\mathscr{O}(D)=H^{\otimes k}$ for some $k>0$, the sheaf $F=J_{1} \oplus J_{2}$ is semistable only if $c_{1}\left(J_{1}(-n)\right) \cdot[D]=c_{1}\left(J_{2}(-n)\right) \cdot[D]=0$. So we have proved

Corollary 1.8. Let $D \in|k H|$. Then one can always descend the line bundle $\mathscr{L}_{\mathscr{Q}}\left(D, \theta_{D}\right)$ on $\mathscr{Q}^{s s}$ to a line bundle on $\mathscr{M}_{d}$. We shall denote the descent line bundle by $\mathscr{L}_{\mathscr{M}}\left(D, \theta_{D}\right)$.

\section{Linear series of $\mathscr{L}_{\mathscr{M}}\left(D, \theta_{D}\right)$ on $\mathscr{M}_{d}$}

Fix $k \geq 1$. Let $D \in|k H|$ be a smooth divisor. In this section we show that the line bundle $\mathscr{L}_{\mathscr{M}}\left(D, \theta_{D}\right)$ on $\mathscr{M}_{d}$ is independent of the choice of $D$ and $\theta_{D}$. We will also show that if $k \geq 2 d+1$ and $m \gg 0$, then the line bundle $\mathscr{L}_{\mathscr{M}}^{\otimes m}\left(D, \theta_{D}\right)=\left(\mathscr{L}_{\mathscr{M}}\left(D, \theta_{D}\right)\right)^{\otimes m}$ is generated by global sections. Therefore, $H^{0}\left(\mathscr{M}_{d}, \mathscr{L}_{\mathscr{M}}^{\otimes m}\left(D, \theta_{D}\right)\right)$ induces a morphism

$$
\gamma: \mathscr{M}_{d} \rightarrow \mathbf{P}\left(H^{0}\left(\mathscr{M}_{d}, \mathscr{L}_{\mathscr{M}}^{\otimes m}\left(D, \theta_{D}\right)\right)^{\vee}\right) .
$$

We use the notation introduced in $\S 1$, and recall that $F_{\mathscr{Q}}$ is the universal quotient sheaf on $X \times \mathscr{Q}$. The immediate goal of this section is to prove the following theorem.

Theorem 2. Let $k \geq 1$, let $D \in|k H|$ be any smooth divisor, and let $\theta_{D} \in \mathrm{Jac}^{g(D)-1}(D)$. Then the line bundle $\mathscr{L}_{\mathscr{M}}\left(D, \theta_{D}\right)$ constructed in Proposition 1.3 is independent of the choice of $D$ and $\theta_{D}$. In other words, there is a line bundle $\mathscr{L}_{\mathscr{M}, k}$ on $\mathscr{M}_{d}$ so that for any pair $\left(D, \theta_{D}\right)$,

$$
\mathscr{L}_{\mathscr{M}, k} \cong \mathscr{L}_{\mathscr{M}}\left(D, \theta_{D}\right) \text {. }
$$

We first study the general situation. Let $S$ be any quasi-projective scheme and let $F_{S}$ be a family of torsion free sheaves on $X \times S$ flat over $S$ having the property that $\operatorname{det} F_{S}=p_{2}^{*} I$, where $I$ is a line bundle on $S$. Let $D \in|k H|$ be any smooth divisor and let $\theta_{0}, \theta_{1} \in \mathrm{Jac}^{g(D)-1}(D)$ be two line bundles on $D$. By Proposition 1.3, to the line bundles $\theta_{i}$ with $i=0,1$, we associate the line bundles $\mathscr{L}_{S}\left(D, \theta_{i}\right)$ on $S$. Now let $\Theta$ be the Poincare line bundle on $D \times \mathrm{Jac}^{g(D)-1}(D)$ and let $K$ be the line bundle $\operatorname{Det}\left(R p_{2, *} \Theta\right)^{\otimes 2}$ on $\operatorname{Jac}^{g(D)-1}(D)=\operatorname{Jac}(D)$. We have the following useful observation. 
Lemma 2.1. For any $\theta_{0}, \theta_{1} \in \mathrm{Jac}^{g(D)-1}(D)$ and any identification $h: K \otimes k\left(\theta_{0}\right) \cong K \otimes k\left(\theta_{1}\right)$, there is an isomorphism

$$
\rho_{h}\left(\theta_{0}, \theta_{1}\right): \mathscr{L}_{S}\left(D, \theta_{0}\right) \stackrel{\cong}{\longrightarrow} \mathscr{L}_{S}\left(D, \theta_{1}\right)
$$

that is functorial under the base change.

Proof. Let $p_{12}$ (resp. $p_{13}, p_{23}$ ) be the projection from $D \times S \times \operatorname{Jac}(D)$ to $D \times S$ (resp. $D \times \operatorname{Jac}(D) ; S \times \operatorname{Jac}(D))$. Consider the sheaf $p_{12}^{*}\left(F_{S \mid D}\right) \otimes$ $p_{13}^{*}(\Theta)$ on $D \times S \times \operatorname{Jac}(D)$, and the corresponding line bundle

$$
\mathscr{L}_{S \times \operatorname{Jac}(D)}=\operatorname{Det}\left(R p_{23, *}\left(p_{12}^{*}\left(F_{S \mid D}\right) \otimes p_{13}^{*}(\Theta)\right)\right)^{-1}
$$

on $S \times \operatorname{Jac}(D)$. By base change property, $\left.\mathscr{L}_{S \times \operatorname{Jac}(D)}\right|_{q_{2}^{-1}\left(\theta_{0}\right)}=\mathscr{L}_{S}\left(D, \theta_{0}\right)$ and $\left.\mathscr{L}_{S \times \mathrm{Jac}(D)}\right|_{q_{2}^{-1}\left(\theta_{1}\right)}=\mathscr{L}_{S}\left(D, \theta_{1}\right)$, where $q_{1}: S \times \mathrm{Jac}(D) \rightarrow S$ and $q_{2}: S \times$ $\operatorname{Jac}(D) \rightarrow \operatorname{Jac}(D)$ are projections. We claim that $\mathscr{L}_{S \times \mathrm{Jac}(D)} \cong q_{1}^{*} L \otimes q_{2}^{*} K$, where $L=\mathscr{L}_{S \times \operatorname{Jac}(D) \mid S \times \theta_{0}}$ is the restriction of $\mathscr{L}_{S \times \operatorname{Jac}(D)}$ to $S \times \theta_{0} \subset$ $S \times \operatorname{Jac}(D)$. Indeed, for any rank-two vector bundle $E$ on $D$ satisfying $\operatorname{det} E=\mathscr{O}$, we can associate the vector bundle $p_{1}^{*} E \otimes \Theta$ on $D \times \operatorname{Jac}(D)$ to a line bundle $\operatorname{Det}\left(R p_{2, *}\left(p_{1}^{*} E \otimes \Theta\right)\right)$ on $\operatorname{Jac}(D)$. Let $J$ be a very ample line bundle on $D$ so that

$$
0 \rightarrow J^{-1} \rightarrow E \rightarrow J \rightarrow 0
$$

Then

$\operatorname{Det}\left(R p_{2, *}\left(p_{1}^{*} E \otimes \Theta\right)\right)=\operatorname{Det}\left(R p_{2, *}\left(p_{1}^{*} J^{-1} \otimes \Theta\right)\right) \otimes \operatorname{Det}\left(R p_{2, *}\left(p_{1}^{*} J \otimes \Theta\right)\right)$.

We further fit $J$ into the exact sequence

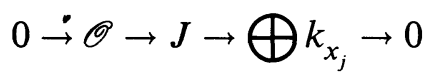

with $\left\{x_{i}\right\} \subset D$ distinct. Then $J^{-1}$ belongs to the exact sequence

$$
0 \rightarrow J^{-1} \rightarrow \mathscr{O} \rightarrow \bigoplus k_{x_{j}} \rightarrow 0
$$

So

$$
\begin{aligned}
\operatorname{Det}\left(R p_{2, *}\left(p_{1}^{*} J \otimes \Theta\right)\right)= & \operatorname{Det}\left(R p_{2, *}\left(p_{1}^{*} \mathscr{\Theta} \otimes \Theta\right)\right) \\
& \otimes \operatorname{Det}\left(R p_{2, *}\left(p_{1}^{*}\left(\bigoplus k_{x_{j}}\right) \otimes \Theta\right)\right) \\
\operatorname{Det}\left(R p_{2, *}\left(p_{1}^{*} J^{-1} \otimes \Theta\right)\right)= & \operatorname{Det}\left(R p_{2, *}\left(p_{1}^{*} \mathscr{O} \otimes \Theta\right)\right) \\
& \otimes \operatorname{Det}\left(R p_{2, *}\left(p_{1}^{*}\left(\bigoplus k_{x_{j}}\right) \otimes \Theta\right)\right)^{-1}
\end{aligned}
$$


Thus,

$$
\begin{aligned}
\operatorname{Det}\left(R p_{2, *}\left(p_{1}^{*} E \otimes \Theta\right)\right) & =\operatorname{Det}\left(R p_{2, *}\left(p_{1}^{*} J^{-1} \otimes \Theta\right)\right) \otimes \operatorname{Det}\left(R p_{2, *}\left(p_{1}^{*} J \otimes \Theta\right)\right) \\
& =\operatorname{Det}\left(R p_{2, *}\left(p_{1}^{*} \mathscr{O} \otimes \Theta\right)\right)^{\otimes 2} .
\end{aligned}
$$

For the moment we assume $s \in S$ is a closed point such that $F_{s \mid D}$ is locally free. Let $V$ be a neighborhood of $s \in S$ so that $F_{V}=F_{S \mid X \times V}$ is locally free at $D \times V$ and that $F_{V \mid D}$ belongs to the exact sequence

$$
0 \rightarrow p_{1}^{*} J^{-1} \rightarrow F_{V \mid D} \rightarrow p_{1}^{*} J \otimes \operatorname{det} F_{V \mid D} \rightarrow 0 .
$$

Then the restriction of the sheaf $\mathscr{L}_{S \times \operatorname{Jac}(D)}$ to $V \times \operatorname{Jac}(D)$ is equal to

$$
\begin{aligned}
\operatorname{Det}( & \left.R p_{23, *}\left(p_{12}^{*} F_{V \mid D} \otimes p_{13}^{*} \Theta\right)\right)^{-1} \\
= & \operatorname{Det}\left(R p_{23, *}\left(p_{1}^{*} J^{-1} \otimes p_{13}^{*} \Theta\right)\right)^{-1} \\
& \otimes \operatorname{Det}\left(R p_{23, *}\left(p_{1}^{*} J \otimes p_{12}^{*} \operatorname{det}\left(F_{V \mid D}\right) \otimes p_{13}^{*} \Theta\right)\right)^{-1} \\
= & q_{1}^{*} I^{\otimes c} \otimes \operatorname{Det}\left(R p_{23, *}\left(p_{1}^{*} J^{-1} \otimes p_{13}^{*} \Theta\right)\right)^{-1} \\
& \otimes \operatorname{Det}\left(R p_{23, *}\left(p_{1}^{*} J \otimes p_{13}^{*} \Theta\right)\right)^{-1} \\
= & q_{1}^{*} I^{\otimes c} \otimes q_{2}^{*} K,
\end{aligned}
$$

where $c$ is an integer. The situation where $F_{s}$ is a rank two nonlocally free sheaf with $s \in S$ can be proved similarly by using the locally free resolution (1.9). Therefore, the line bundle $\mathscr{L}_{S \times \mathrm{Jac}(D)} \otimes q_{2}^{*} K^{-1}$ is a pullback line bundle from a line bundle $L$ on $S$. So

$$
\mathscr{L}_{S \times \operatorname{Jac}(D)}=p_{1}^{*} L \otimes p_{2}^{*} K .
$$

Finally we remark that $(2.4)$ is canonical under the base change. Now if we specify an identification $h: K \otimes k\left(\theta_{0}\right) \cong K \otimes k\left(\theta_{1}\right)$, thanks to (2.4), there is a unique isomorphism

$$
\rho_{h}\left(\theta_{0}, \theta_{1}\right): \mathscr{L}_{S}\left(D, \theta_{0}\right) \cong \mathscr{L}_{S}\left(D, \theta_{1}\right)
$$

that is functorial under the base change. q.e.d.

Now we study the case where two different $D_{0}, D_{1} \in|k H|$ are involved. Let $D_{t}$ be a linear series of divisors in $|k H|$ containing $D_{0}$ and $D_{1}$ as its members. Parallel to the proof of Lemma 1.2, we let $D_{t}$ be the linear series in $|k H|$ that contains $D_{0}$ and $D_{1}$ and let $Y$ be the blowing-up of $X$ along base points of the linear series $D_{t}$. Let $Q_{1}$ and $Q_{2}$ be locally free sheaves on $X \times S$ satisfying (1.9) and let $\tilde{p}^{*} Q_{1}$ and $\tilde{p}^{*} Q_{2}$ be 
corresponding locally free sheaves on $Y \times S$ (see Lemma 1.2 for notation). Then we form the determinant line bundle

$$
\mathscr{L}_{\mathbf{P}^{1} \times S}=\operatorname{Det}\left(R f_{*}\left(\tilde{p}^{*} Q_{1} \otimes p^{*} \theta\right)\right)^{-1} \otimes \operatorname{Det}\left(R f_{*}\left(\tilde{p}^{*} Q_{2} \otimes p_{1}^{*} \theta\right)\right)
$$

on $\mathbf{P}^{1} \times S$, where $\theta$ is a line bundle on $Y$ with $\operatorname{deg}\left(\theta_{\mid D_{0}}, D_{0}\right)=g\left(D_{0}\right)-1$. Thanks to the cohomology and base change theorem [10, III, 12.11], the line bundle $\mathscr{L}_{\mathbf{P}^{1} \times S}$ must be of the form

$$
\mathscr{L}_{\mathbf{P}^{1} \times S}=p_{1}^{*} \mathscr{O}(\alpha) \otimes p_{2}^{*} L
$$

where $\mathscr{O}(\alpha)$ is a line bundle on $\mathbf{P}^{1}$, and $L$ is a line bundle on $S$. One checks directly that $\alpha$ is an integer depending only on the second Chern class of the family $F_{S}$. Finally, one notes that this construction is canonical. So.we have proved the following lemma.

Lemma 2.2. Let $D_{0}, D_{1} \in|k H|$ be two smooth divisors and let $\theta_{i} \in$ $\operatorname{Jac}^{g\left(D_{i}\right)-1}\left(D_{i}\right)$ with $i=0,1$ be any line bundles. Then there is an isomorphism

$$
\rho\left(D_{0}, \theta_{0} ; D_{1}, \theta_{1}\right): \mathscr{L}_{S}\left(D_{0}, \theta_{0}\right) \rightarrow \mathscr{L}_{S}\left(D_{1}, \theta_{1}\right)
$$

which is functorial under the base change.

Proof. Clearly for $t \in \mathbf{P}^{1}$ and $D_{t}$ smooth, $\mathscr{L}_{\mathbf{P}^{1} \times S \mid\{t\} \times S} \cong \mathscr{L}_{S}\left(D_{t}, \theta_{\mid D_{t}}\right)$. If we denote by $0,1 \in \mathbf{P}^{1}$ the points corresponding to $D_{0}$ and $D_{1}$ in the linear series $D_{t}$, then by (2.6) there is an isomorphism $\rho\left(D_{0}, \theta_{0} ; D_{1}, \theta_{1}\right)$ which is uniquely determined if we specify an identification $h: \mathscr{O}(\alpha) \otimes$ $k(0) \cong \mathscr{O}(\alpha) \otimes k(1)$. Since $\alpha$ depends only on the second Chern class of the family, $h$ can be chosen independent of the particular families. Since the decomposition (2.6) is functorial under the base change, the isomorphism (2.7) is functorial under the base change also. q.e.d.

Combining these two lemmas gives the following general result.

Proposition 2.3. Assume that $S$ is a quasi-projective scheme and that $F_{S}$ is any family of rank two torsion free sheaves on $X \times S$ flat over $S$ such that $\operatorname{det} F_{S}=p_{2}^{*} I$, where $I$ is a line bundle on $S$ and $c_{2}\left(F_{s}\right)=d$ for closed $s \in S$. Let $k \geq 1$, let $D_{0}, D_{1} \in|k H|$ be any smooth divisors, and let $\theta_{0} \in \mathrm{Jac}^{g\left(D_{0}\right)-1}\left(D_{0}\right)$ and $\theta_{1} \in \mathrm{Jac}^{g\left(D_{1}\right)-1}\left(D_{1}\right)$ be any line bundles. Then based on the data depending only on $d$, described in Lemmas 2.1 and 2.2, there is a unique isomorphism

$$
\rho\left(D_{0}, \theta_{0} ; D_{1}, \theta_{1}\right): \mathscr{L}_{S}\left(D_{0}, \theta_{0}\right) \rightarrow \mathscr{L}_{S}\left(D_{1}, \theta_{1}\right)
$$

which is functorial under the base change. 
We are ready to prove Theorem 2. Let $\mathscr{Q}^{s s} \subset$ Quot $_{\mathscr{Q}^{N}}^{d, 0}$ be as before and let $F_{\mathscr{Q}}$ be the restriction of the universal quotient sheaf to $X \times \mathscr{Q}^{s s}$. For any smooth $D \in|k H|$ and $\theta_{D} \in \operatorname{Jac}^{g(D)-1}(D)$, define

$$
\mathscr{L}_{\mathscr{Q}}\left(D, \theta_{D}\right)=\operatorname{Det}\left(R p_{2, *} F_{\mathscr{Q}}(-n)_{\mid D} \otimes p_{1}^{*} \theta_{D}\right)^{-1} \text {. }
$$

Now given pairs $\left(D_{i}, \theta_{i}\right)$ with $D_{i} \in|k H|$ and $\theta_{i} \in \mathrm{Jac}^{g\left(D_{i}\right)-1}\left(D_{i}\right), i=$ 0,1 , there are corresponding line bundles $\mathscr{L}_{\mathscr{Q}}\left(D_{i}, \theta_{i}\right)$ on $\mathscr{Q}^{s s}$ and descent line bundles $\mathscr{L}_{\mathscr{M}}\left(D_{i}, \theta_{i}\right)$ on $\mathscr{M}_{d}$. Thanks to Proposition 2.3 , there is a unique isomorphism (depending on some specific data)

$$
k\left(D_{0}, \theta_{0} ; D_{1}, \theta_{1}\right): \mathscr{L}_{\mathscr{Q}}\left(D_{0}, \theta_{0}\right) \rightarrow \mathscr{L}_{\mathscr{Q}}\left(D_{1}, \theta_{1}\right) .
$$

To show that this isomorphism can be descended to an isomorphism $\rho_{\mathscr{M}}\left(D_{0}, \theta_{0} ; D_{1}, \theta_{1}\right)$ between $\mathscr{L}_{\mathscr{M}}\left(D_{0}, \theta_{0}\right)$ and $\mathscr{L}_{\mathscr{M}}\left(D_{1}, \theta_{1}\right)$, we need to show that the isomorphism $\rho\left(D_{0}, \theta_{0} ; D_{1}, \theta_{1}\right)$ in $(2.10)$ is $\mathscr{G}$-equivariant. Recall that through the surjection $\operatorname{GL}(N, \mathbb{C}) \rightarrow \operatorname{PGL}(N, \mathbb{C}), \mathscr{Q}^{s s}$ is a $\mathrm{GL}(N, \mathbb{C})$-scheme and, further, $F_{\mathscr{Q}}$ admits a $\mathrm{GL}(N, \mathbb{C})$-linearization. Let $g \in \mathrm{GL}(N, \mathbb{C})$ and let $g_{*}$ be the action on $F_{\mathscr{Q}}$ induced by the $\operatorname{GL}(N, \mathbb{C})$ linearization. Then, since the isomorphism $\rho\left(D_{0}, \theta_{0} ; D_{1}, \theta_{1}\right)$ is functorial under the base change,

$$
\rho\left(D_{0}, \theta_{0} ; D_{1}, \theta_{1}\right) \circ \operatorname{det}\left(g_{* \mid D_{0}}\right)=\operatorname{det}\left(g_{* \mid D_{1}}\right) \circ \rho\left(D_{0}, \theta_{0} ; D_{1}, \theta_{1}\right) .
$$

Therefore, $\rho\left(D_{0}, \theta_{0} ; D_{1}, \theta_{1}\right)$ is $\mathscr{G}$-equivariant. In particular, it descends to an isomorphism between $\mathscr{L}_{\mathscr{M}}\left(D_{0}, \theta_{0}\right)$ and $\mathscr{L}_{\mathscr{M}}\left(D_{1}, \theta_{1}\right)$. In the following, we will denote this unique line bundle by $\mathscr{L}_{\mathscr{M}, k}$. q.e.d.

Next, we will study the space $H^{0}\left(\mathscr{M}_{d}, \mathscr{L}_{\mathscr{M}, k}^{\otimes m}\right)$ when $m$ is sufficiently large. We begin our discussion with any quasi-projective scheme $S$ coupled with a flat family of torsion free sheaves $F_{S}$ on $X \times S$ with $\operatorname{det} F_{S}=$ $p_{2}^{*} I$. Let $S(D) \subset S$ be the open subset consisting of points $s \in S$ where $F_{s \mid D}$ is locally free and semistable. The restriction of $F_{S}$ to $D \times S(D)$ induces a morphism

$$
j_{D}: V_{S}(D) \rightarrow \mathscr{M}(D),
$$

where $\mathscr{M}(D)$ is the moduli scheme of rank-two semistable vector bundles $E$ on $D$ with $\operatorname{det} E=\mathscr{O}$. On $\mathscr{M}(D)$, there is a similar line bundle $\mathscr{L}_{D}$ which is the descent of the dual of a determinant line bundle on a properly chosen Grothendieck quotient scheme. We describe the construction of this quotient scheme and $\mathscr{L}_{D}$ briefly. Let $L$ be a very ample line bundle on $D$ so that for any rank-two semistable bundle $E$ on $D$ with $\operatorname{deg} E=$ $\mathscr{O}, h^{1}(L \otimes E)=0$ and $H^{0}(L \otimes E)$ generates $L \otimes E$. Let $2 l=h^{0}(L \otimes E)$ and 
let $W=\bigoplus^{2 l} L^{-1}$. Then any semistable vector bundle on $D$ corresponds to a unique $\operatorname{SL}(2 l, \mathbb{C})$ orbit in $\operatorname{Quot}_{W}^{0}$, where Quot $_{W}^{0}$ is the Grothendieck quotient scheme parametrizing all rank-two quotient sheaves $E$ of $W$ with $\operatorname{det} E=\mathscr{O}$. Let $\mathscr{R} \subset$ Quot $_{W}^{0}$ be the open subset consisting of those quotient sheaves $W \rightarrow E$ such that $H^{0}(W \otimes L) \rightarrow H^{0}(E \otimes L)$ is an isomorphism. Then, similar to the surfaces case, there is a Gieseker immersion

$$
\mu_{D}: \mathscr{R} \rightarrow \mathbf{P}\left(\operatorname{Hom}\left(\bigwedge^{2} \mathbb{C}^{2 l}, H^{0}\left(L^{\otimes 2}\right)\right)\right) .
$$

In the following, we abbreviate $\mathscr{W}=\operatorname{Hom}\left(\bigwedge^{2} \mathbb{C}^{2 l}, H^{0}\left(L^{\otimes 2}\right)\right)$ and $\mathscr{G}^{\prime}=$ $\mathrm{SL}(2 l, \mathbb{C})$. Note that $\mathbf{P}(\mathscr{W})$ is a $\mathscr{G}^{\prime}$-scheme and $\mu_{D}$ is a $\mathscr{G}^{\prime}$-morphism. Further, if we let $\mathscr{R}^{s s} \subset$ Quot $_{W}^{0}$ be the set of semistable quotient sheaves, then $E \in \mathscr{R}^{s s}$ if and only if $\mu_{D}(E)$ is semistable under $\mathscr{G}^{\prime}$. Therefore,

$$
\mathscr{R}^{s s} / / \mathscr{G}^{\prime}=\mathscr{M}(D)
$$

is a subscheme of $\mathbf{P}(W)^{s s} / / \mathscr{G}^{\prime}$. Now, if we let $E_{\mathscr{R}}$ be the universal quotient sheaf on $D \times \mathscr{R}$ and let $\theta_{D} \in \mathrm{Jac}^{g(D)-1}(D)$ be any line bundle, then, by using the descent lemma, the restriction to $\mathscr{R}^{s s}$ of the determinant line bundle

$$
\operatorname{Det}\left(R p_{2, *}\left(E_{\mathscr{R}} \otimes p_{1}^{*} \theta_{D}\right)\right)
$$

descends to a line bundle on $\mathscr{M}(D)$. We denote the dual of (2.13) by $\mathscr{L}_{\mathscr{R}}\left(\theta_{D}\right)$ and denote the descent of $\mathscr{L}_{\mathscr{R}}\left(\theta_{D}\right)$ by $\mathscr{L}_{D}\left(\theta_{D}\right)$. Note that there is a canonical $\mathscr{G}^{\prime}$-linearization of the hyperplane line bundle $\mathscr{O}_{\mathbf{P}}(1)$ on $\mathbf{P}(\mathscr{W})$ induced by the canonical $\mathrm{GL}(\mathscr{W}, k)$-linearization of $\mathscr{O}_{\mathbf{P}}(1)$. We have the following observation made by Donaldson.

Proposition 2.4 (Donaldson). Let $\mathscr{O}_{\mathbf{P}}(1)$ be the hyperplane line bundle on $\mathbf{P}(\mathscr{W})$. Then there is an isomorphism

$$
\left.\mathscr{L}_{\mathscr{R}}\left(\theta_{D}\right) \cong \mu_{D}^{*} \mathscr{O}_{\mathbf{P}}(l)\right|_{\mathscr{R}},
$$

which is $\mathscr{G}^{\prime}$-equivariant.

Proof. The proof given by Donaldson for the case $\theta_{D}^{\otimes 2}=K_{D}$ and for the family of semistable vector bundles works for any $\theta_{D} \in \mathrm{Jac}^{g(D)-1}(D)$ and any family of sheaves on $\mathscr{R}$ without any change (see [3, Proposition $5.4]$ or $[7, \S 3])$. q.e.d.

Now let us recall the geometric invariant theory. There is an integer $m_{0}$ such that the line bundle $\mathscr{O}_{\mathbf{P}}\left(m_{0} l\right)$ descends to ample line bundle $\mathscr{O}_{\mathbf{P} / \mathscr{G}^{\prime}}\left(m_{0} l\right)$ over $\mathbf{P}(\mathscr{W})^{s s} / / \mathscr{G}^{\prime}$. For sufficiently large $m, m_{0} \mid m$, the set of 
$\mathscr{G}^{\prime}$-invariant sections $H^{0}\left(\mathscr{W}, \mathscr{O}_{\mathbf{P}}(m l)\right)^{\mathscr{I}^{\prime}} \subset H^{0}\left(\mathscr{W}, \mathscr{O}_{\mathbf{P}}(m l)\right)$ is nonempty. Further, an element $x \in \mathbf{P}(\mathscr{W})$ is semistable if and only if there is a $v \in H^{0}\left(\mathscr{W}, \mathscr{O}_{\mathbf{P}}(m l)\right)^{\mathscr{S}^{\prime}}$ such that $v(x) \neq 0$. Consequently, the geometric invariant theory quotient $\mathbf{P}(\mathscr{W})^{s s} / / \mathscr{G}^{\prime}$ is defined to be the image scheme of $\mathbf{P}(\mathscr{W})^{s s}$ in $\mathbf{P}\left(\left(H^{0}\left(\mathscr{W}, \mathscr{O}_{\mathbf{P}}(m l)\right)^{\mathscr{G}^{\prime}}\right)\right)$. Therefore, $H^{0}\left(\mathbf{P}(\mathscr{W}), \mathscr{O}_{\mathbf{P}}(m l)\right)^{\mathscr{G}^{\prime}}$ is canonically contained in $H^{0}\left(\mathbf{P}(\mathscr{W})^{s s} / / \mathscr{G}^{\prime}, \mathscr{O}_{\mathbf{P} / \mathscr{G}^{\prime}}(m l)\right)$. Note that $\mathscr{O}_{\mathbf{P} / \mathscr{G}^{\prime}}(m l)_{\mid \mathscr{M}(D)}=\left(\mathscr{L}_{D}\left(\theta_{D}\right)\right)^{\otimes m}=\mathscr{L}_{D}^{\otimes m}\left(\theta_{D}\right)$. We denote by $V_{D}(m) \subset$ $H^{0}\left(\mathscr{M}(D), \mathscr{L}_{D}^{\otimes m}\left(\theta_{D}\right)\right)$ the image of

$$
H^{0}\left(\mathscr{W}, \mathscr{O}_{\mathbf{P}}(m l)\right)^{\mathscr{G}^{\prime}} \rightarrow H^{0}\left(\mathscr{M}(D), \mathscr{L}_{D}^{\otimes m}\left(\theta_{D}\right)\right) .
$$

For simplicity, in the remainder of this paper, by $m \gg 0$ we always mean $m \gg 0$ and $m_{0} \mid m$.

Returning to the sheaf $F_{S}$ on $X \times S$ flat over $S$, since $F_{S}$ is a flat family of torsion free sheaves, the restriction of it to $D \times S$, say $F_{S \mid D}$, is a family of sheaves on $D \times S$ flat over $S$. Since $S$ is quasi-projective, we can choose $L$ to be a sufficiently ample line bundle on $D$ so that for any $s \in S, h^{1}\left(F_{s \mid D} \otimes L\right)=0$ and $H^{0}\left(F_{s \mid D} \otimes L\right)$ generates the sheaf $F_{s \mid D}$. We still denote $2 l=h^{0}\left(F_{s \mid D} \otimes L\right)$. Now we cover $S$ by affine open sets $T_{1}, \cdots, T_{n}$ so that on each $T_{i}, F_{T_{i}}=F_{S \mid X \times T_{i}}$, there is an isomorphism

$$
\eta_{i}: p_{2, *}\left(F_{T_{i} \mid D} \otimes p_{1}^{*} L\right) \cong \bigoplus^{2 l} \mathscr{O}_{T_{i}}
$$

Then the homomorphism $\eta_{i}$ induces morphism

$$
\bar{\eta}_{i}: T_{i} \rightarrow \operatorname{Quot}_{W}^{0}
$$

Clearly, $\bar{\eta}_{i}\left(T_{i}\right) \subset \mathscr{R}$. Further, since both $\mathscr{L}_{S}\left(D, \theta_{D}\right)_{\mid T_{i}}$ and $\bar{\eta}_{i}^{*} \mathscr{L}_{\mathscr{R}}\left(\theta_{D}\right)$ are duals of the determinant line bundles of isomorphic complexes, we have

$$
\bar{\eta}_{i}^{*} \mathscr{L}_{\mathscr{R}}\left(\theta_{D}\right) \cong \mathscr{L}_{S}\left(D, \theta_{D}\right)_{\mid T_{i}}
$$

If we restrict isomorphism (2.17) to $T_{i} \cap S(D)$, then, since $\mathscr{L}_{\mathscr{R}}\left(\theta_{D}\right)_{\mid \mathscr{R}^{s s}}$ descends to the line bundle $\mathscr{L}_{D}\left(\theta_{D}\right)$, we conclude that $\left\{\left.\bar{\eta}_{i}^{*}\right|_{T_{i} \cap S(D)}\right\}$ provides an isomorphism

$$
j_{D}^{*} \mathscr{L}_{D}\left(\theta_{D}\right) \cong \mathscr{L}_{S}\left(D, \theta_{D}\right)_{\mid S(D)} .
$$

Thus (2.18) induces a homomorphism

$$
j_{D}^{*}: V_{D}(m) \rightarrow H^{0}\left(S(D), \mathscr{L}_{S}^{\otimes m}\left(D, \theta_{D}\right)\right) .
$$


We claim that there is a canonical homomorphism

$$
\rho_{D}: V_{D}(m) \rightarrow H^{0}\left(S, \mathscr{L}_{S}^{\otimes m}\left(D, \theta_{D}\right)\right)
$$

extending $j_{D}^{*}$ and having the property that for any $v \in V_{D}(m), \rho_{D}(v)(F)$ $=0$ if $F \notin S(D)$. Indeed, by Proposition 2.4, we have

$$
\begin{aligned}
\bar{\eta}_{i}^{*} \circ \mu_{D}^{*} \mathscr{O}_{\mathbf{P}}(l) & =\bar{\eta}_{i}^{*} \operatorname{Det}\left(R p_{2, *} E_{\mathscr{R}} \otimes p_{1}^{*} \theta_{D}\right)^{-1}, \\
& =\operatorname{Det}\left(R p_{2, *} F_{T_{i} \mid D} \otimes p_{1}^{*} \theta_{D}\right)^{-1} .
\end{aligned}
$$

Let $v \in V_{D}(m)$ be any section. There is a $\mathscr{G}^{\prime}$-invariant section $\bar{v} \in$ $H^{0}\left(\mathbf{P}(\mathscr{W}), \mathscr{O}_{\mathbf{P}}(m l)\right)^{\mathscr{G}^{\prime}}$ which descends to $v$. Define $\bar{\eta}_{i}^{*} \circ \mu_{D}^{*}(\bar{v})$ to be the pullback section in $H^{0}\left(T_{i}, \mathscr{L}_{S}^{\otimes m}\left(D, \theta_{D}\right)_{\mid T_{i}}\right)$. We claim that on $T_{i} \cap$ $T_{j} \neq \varnothing, \bar{\eta}_{i}^{*} \circ \mu_{D}^{*}(\bar{v})=\bar{\eta}_{j}^{*} \circ \mu_{D}^{*}(\bar{v})$. Indeed, since both $\eta_{i}$ and $\dot{\eta}_{j}$ are isomorphisms and $\left.F_{T_{i}}\right|_{T_{i} \cap T_{j}}=\left.F_{T_{j}}\right|_{T_{i} \cap T_{j}}$, there is an isomorphism

$$
g: \bigoplus^{2 l} \mathscr{O}_{T_{i} \cap T_{j}} \rightarrow \bigoplus^{2 l} \mathscr{O}_{T_{i} \cap T_{j}}
$$

over $T_{i} \cap T_{j}$ so that $\eta_{i}=g \circ \eta_{j}$. Note that each isomorphism $\bar{\eta}_{i}^{*}$ depends canonically on $\eta_{i}$, thus $\eta_{i}^{*} \circ g_{*}=\bar{\eta}_{j}^{*}$, where $g_{*}$ is induced by the $\mathscr{G}^{\prime}$ linearization of the line bundle $\mathscr{L}_{\mathscr{R}}\left(\theta_{D}\right)$. Since $\bar{v}$ is $\mathscr{G}^{\prime}$-invariant and $\mu_{D}$ is $\mathscr{G}^{\prime}$-equivariant, $\mu_{D}^{*}(\bar{v})$ is $\mathscr{G}^{\prime}$-invariant. Thus, $g_{*} \circ \mu_{D}^{*}(\bar{v})=\mu_{D}^{*}(\bar{v})$ and then $\bar{\eta}_{i}^{*} \circ \mu_{D}^{*}(\bar{v})=\bar{\eta}_{j}^{*} \circ \mu_{D}^{*}(\bar{v})$. Therefore, the local sections $\left\{\bar{\eta}_{i}^{*} \circ \mu_{D}^{*}(\bar{v})\right\}$ glue together to form a global section $\rho_{D}(v)$ on $S$. We remark that for closed $s \in S, \rho_{D}(v)(s)=0$ if $s \in T_{i}$ and $\bar{v}\left(\bar{\eta}_{i}(s)\right)=0$. This is exactly the case where either $F_{s \mid D}$ is not semistable or $v\left(F_{s \mid D}\right)=0$. Thus we proved the following proposition.

Proposition 2.5. Let $k \geq 1$. For any smooth $D \in|k H|$ and any section $v \in V_{D}(m) \subset H^{0}\left(\mathscr{M}(D), \mathscr{L}_{D}^{\otimes m}\right)$, the pullback section $j_{D}^{*}(v) \in$ $H^{0}\left(S(D), \mathscr{L}_{S}^{\otimes m}\left(D, \theta_{D}\right)\right)$ from (2.18) can be extended over $S$ to a section $\tilde{v} \in H^{0}\left(S, \mathscr{L}_{S}^{\otimes m}\left(D, \theta_{D}\right)\right)$. Furthermore,

$$
S \backslash S(D) \subset \tilde{v}^{-1}(0) .
$$

We remark that the subset $V_{D}(m) \subset H^{0}\left(\mathscr{M}(D), \mathscr{L}_{D}^{\otimes m}\right)$ may depend on the choice of the ample line bundle $L$ on $D$. But this is irrelevant to our study as long as the linear series $V_{D}(m)$ provides a projective immersion of $\mathscr{M}(D)$.

Theorem 3. Let $k \geq 1$ and let $\mathscr{L}_{\mathscr{M}, k}$ be the line bundle provided by Theorem 2. Then for any smooth $D \in|k H|$ and $m \gg 0$, there is a 
homomorphism

$$
\rho_{D}: V_{D}(m) \rightarrow H^{0}\left(\mathscr{M}_{d}, \mathscr{L}_{\mathscr{M}, k}^{\otimes m}\right)
$$

induced by the rational map $j_{D}: \mathscr{M}_{d} \rightarrow \mathscr{M}(D)$ and the isomorphism $j_{D}^{*} \mathscr{L}_{D} \cong \mathscr{L}_{\mathscr{M}, k}$ defined on the open set where $j_{D}$ is a morphism. Further, if $k \geq 2 d+1$, then, for any closed point $E \in \mathscr{M}_{d}$ and generic $D \in|k H|, E$ is not the base point of $\rho_{D}\left(V_{D}(m)\right)$. In particular, if we let $\mathscr{V}_{D}^{\mathscr{M}}(m)=\rho_{D}\left(V_{D}(m)\right)$ and let $\mathscr{V}_{k}^{M}(m)$ be the linear span of all $\mathscr{V}_{D}^{\mathscr{M}}(m) \subset H^{0}\left(\mathscr{M}_{d}, \mathscr{L}_{\mathscr{M}, k}^{\otimes m}\right)$ with smooth $D \in|k H|$, then $\mathscr{V}_{k}^{\mathscr{M}}(m)$ is base point free.

Proof. Recall that $\mathscr{L}_{\mathscr{M}, k}$ is the descent of the line bundle $\mathscr{L}_{\mathscr{Q}}\left(D, \theta_{D}\right)$ with $D \in|k H|$ and $\theta_{D} \in \mathrm{Jac}^{g(D)-1}(D)$. If we apply Proposition 2.5 to the quasi-projective scheme $\mathscr{Q}^{s s} \subset \mathscr{Q}$ and the sheaf $F_{\mathscr{Q}}(-n)$ on $X \times \mathscr{Q}^{s s}$, then we obtain a rational map $j_{D}^{\mathscr{Q}}: \mathscr{Q}^{s s} \rightarrow \mathscr{M}(D)$, the isomorphism

$$
j_{D}^{\mathscr{Q}, *} \mathscr{L}_{D}\left(\theta_{D}\right) \cong \mathscr{L}_{\mathscr{Q}}\left(D, \theta_{D}\right)_{\mid \mathscr{Q}^{s s}(D)}
$$

and the homomorphism

$$
\rho_{D}^{\mathscr{Q}}: V_{D}(m) \rightarrow H^{0}\left(\mathscr{Q}^{s s}, \mathscr{L}_{\mathscr{Q}}^{\otimes m}\left(D, \theta_{D}\right)\right)
$$

It is clear that to show $j_{D}^{*} \mathscr{L}_{D}\left(\theta_{D}\right) \cong \mathscr{L}_{\mathscr{M}, k}$ and

$$
\rho_{D}: V_{D}(m) \rightarrow H^{0}\left(\mathscr{M}_{d}, \mathscr{L}_{\mathscr{M}}^{\otimes m}\left(D, \theta_{D}\right)\right)
$$

exist, it suffices to show that $j_{D}^{\mathscr{Q}, *}$ and the image $\rho_{D}^{\mathscr{Q}}\left(V_{D}(m)\right)$ are $\mathscr{G}^{\prime}$ invariant. That is apparent since the constructions are canonical. So we have proved that such a homomorphism exists.

In the case $k \geq 2 d+1$, Bogomolov's theorem states that if $E$ is any $\mu$-semistable vector bundle with $c_{2}(E) \leq d$ and $D \in|k H|$ is any smooth divisor, then $E_{\mid D}$ is necessarily semistable. Thus to any closed point $F \in$ $\mathscr{M}_{d}, F_{\mid D}$ is semistable for generic $D \in|k H|$. Choose $v \in V_{D}(m)$ so that $v\left(F_{\mid D}\right) \neq 0$; then $\rho_{D}(v)(F) \neq 0$. Hence we have established the theorem.

Remark. It is unclear whether the meromorphic sections of $\mathscr{L}_{\mathscr{M}, k}^{\otimes m}$ which are pullback sections from $H^{0}\left(\mathscr{M}(D), \mathscr{L}_{D}^{\otimes m}\right)$ via $j_{D}: \mathscr{M}_{d} \rightarrow \mathscr{M}_{(D)}$ can always be extended to holomorphic sections of $\mathscr{L}_{\mathscr{M}, k}^{\otimes m}$ over $\mathscr{M}_{d}$. When $H$ is fixed, it is proved that when $d$ is sufficiently large, $\mathscr{M}_{d}$ is normal. Thus such a homomorphism $\rho_{D}: H^{0}\left(\mathscr{M}(D), \mathscr{L}_{\mathscr{M}, k}^{\otimes m}\right)$ does exist [13]. 


\section{The canonical points of $\gamma\left(\mathscr{M}_{d}\right)$}

In this section, we will use the linear series $\mathscr{V}_{k}(m) \subset H^{0}\left(\mathscr{M}_{d}, \mathscr{L}_{\mathscr{M}, k}^{\otimes m}\right)$ produced in Theorem 3 to introduce a new scheme $\gamma\left(\mathscr{M}_{d}\right)$. We will then give a complete geometric description of the closed points of $\gamma\left(\mathscr{M}_{d}\right)$.

Parallel to the discussion of $\S 1$, we denote by $\mathscr{E}_{d}^{\mu}(n)$ the set of $\mu$ semistable rank two sheaves $F$ with $\operatorname{det} F=H^{\otimes 2 n}$ and $c_{2}\left(F \otimes H^{-n}\right)=d$. Choose $n \gg 0$ such that for any $F \in \mathscr{E}_{d}^{\mu}(n), H^{i}(F)=\{0\}$ for $i>0$ and $F$ is generated by global sections $H^{0}(F)$. Let $N=\chi(F)$ and let Quot $_{\mathscr{O}^{N}}^{d, 0}$ be the Grothendieck quotient scheme parametrizing all quotient sheaves $F$ of $\bigoplus^{N} \mathscr{O}$ with $\operatorname{det} F=H^{\otimes 2 n}$ and $c_{2}\left(F \otimes H^{-n}\right)=d$. Let $F_{\mathscr{Q}}$ be the universal quotient sheaf on $\mathscr{Q}^{\mu}$, where $\mathscr{Q}^{\mu} \subset \mathscr{Q}$ is the open set consisting of all $\mu$-semistable quotient sheaves. We then let $k>2 d+1$ be a sufficiently large integer so that whenever $E_{1} \in \mathscr{E}_{d_{1}}^{\mu}(n)$ and $E_{2} \in \mathscr{E}_{d_{2}}^{\mu}(n)$ are locally free sheaves with $d_{1}, d_{2} \leq d$, then $h^{j}\left(X, E_{1}^{\vee} \otimes E_{2}(-k)\right)=0$ for $j=0,1$. We will fix such a $k$ once and for all. Finally, we denote by

$$
\mathscr{L}_{\mathscr{Q}}\left(D, \theta_{D}\right)=\operatorname{Det}\left(R p_{2, *} F_{\mathscr{Q}}(-n)_{\mid D} \otimes p_{1}^{*} \theta_{D}\right)^{-1}
$$

the line bundle on $\mathscr{Q}^{\mu}$ where $D \in|k H|$ and $\theta_{D} \in \mathrm{Jac}^{g(D)-1}(D)$. If we apply Propositions 2.3 and 2.5 directly to $\mathscr{Q}^{\mu}$ and the sheaf $F_{\mathscr{Q}}$, we have

Proposition 3.1. With the notation as above, the following hold.

(1) The line bundles $\mathscr{L}_{\mathscr{Q}}\left(D, \theta_{D}\right)$ in (3.1) are independent of the choice of smooth $D \in|k H|$ and $\theta_{D} \in \mathrm{Jac}^{g(D)-1}(D)$. We denote this unique line bundle by $\mathscr{L}_{\mathscr{Q}, k}$.

(2) For any smooth $D \in|k H|$ and $m \gg 0$, there is a homomorphism

$$
\tilde{\rho}_{D}: V_{D}(m) \rightarrow H^{0}\left(\mathscr{Q}^{\mu}, \mathscr{L}_{\mathscr{Q}, k}^{\otimes m}\right),
$$

which is defined by applying Proposition 2.5 to $\mathscr{Q}^{\mu}$ and $F_{\mathscr{Q}^{\mu}}$. We denote the image by $\mathscr{V}_{D}^{\mathscr{Q}}(m)$.

(3) Let $\mathscr{G}=\operatorname{PGL}(N, \mathbb{C})$. Then $\mathscr{Q}^{\mu}$ is $\mathscr{G}$-invariant. Furthermore, $\mathscr{L}_{\mathscr{Q}, k}$ is a $\mathscr{G}$-bundle and $\mathscr{V}_{D}^{\mathscr{Q}}(m) \subset H^{0}\left(\mathscr{Q}^{\mu}, \mathscr{L}_{\mathscr{Q}, k}^{\otimes m}\right)^{\mathscr{G}}$.

(4) With $m \gg 0$, for any closed $s \in \mathscr{Q}^{\mu}$, there is a smooth $D \in|k H|$ such that $s$ is not the base point of the linear series $\mathscr{V}_{D}^{\mathscr{Q}}(m)$.

It is evident that the restrictions of the sections $\mathscr{V}_{D}^{\mathscr{Q}}(m)$ to $\mathscr{Q}^{s s} \subset \mathscr{Q}^{\mu}$ descend to sections in $\mathscr{V}_{D}^{\mathscr{K}}(m) \subset H^{0}\left(\mathscr{M}_{d}, \mathscr{L}_{\mathscr{M}, k}^{\otimes m}\right)$. If we let $\mathscr{V}_{k}^{\mathscr{Q}}(m) \subset$ 
$H^{0}\left(\mathscr{Q}^{u}, \mathscr{L}_{\mathscr{Q}, k}^{\otimes m}\right)$ be the space spanned by all $\mathscr{V}_{D}^{\mathscr{Q}}(k)$ with $D \in|k H|$, then there is a canonical surjective homomorphism $\mathscr{V}_{k}^{\mathscr{Q}}(m) \rightarrow \mathscr{V}_{k}^{M}(m)$. Finally we choose $m$ sufficiently large so that for any smooth $D \in|k H|$, the linear series $V_{D}(m)$ provides an immersion $\mathscr{M}(D) \rightarrow \mathbf{P}\left(V_{D}(m)^{\vee}\right)$. Now let $\gamma$ (resp. $\gamma_{\mathscr{Q}}$ ) be the morphism $\gamma: \mathscr{M}_{d} \rightarrow \mathbf{P}\left(\mathscr{V}_{k}^{\mathscr{M}}(m)^{v}\right)$ (resp. $\left.\gamma_{\mathscr{Q}}: \mathscr{Q}^{\mu} \rightarrow \mathbf{P}\left(\mathscr{V}_{k}^{\mathscr{Q}}(m)^{\mathrm{v}}\right)\right)$. Let $V$ be the kernel of $\mathscr{V}_{k}^{\mathscr{Q}}(m) \rightarrow \mathscr{V}_{k}^{\mu}(m)$ and let $\mathscr{V}_{k}^{\mathscr{Q}}(m) \rightarrow V$ be a homomorphism that is the identity when restricted to $V \subset \mathscr{V}_{k}^{\mathscr{Q}}(m)$. Then we have the isomorphism $\mathscr{V}_{k}^{\mathscr{Q}}(m) \cong \mathscr{V}_{k}^{k}(m) \oplus V$ and the following diagram:

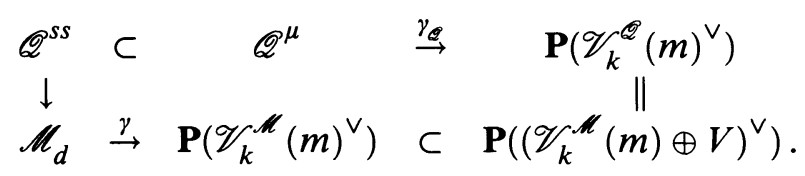

Lemma 3.2. With the notation as above, $\gamma_{\mathscr{Q}}$ and $\gamma$ are morphisms making the diagram (3.2) commutative. Furthermore, $\gamma_{\mathscr{Q}}\left(\mathscr{Q}^{\mu} \cap \overline{\mathscr{Q}}^{s s}\right)$ is identical to $\gamma\left(\mathscr{M}_{d}\right)$ as sets, where $\widetilde{Q}^{s s}$ is the closure of $\mathscr{Q}^{s s}$ in $\mathscr{Q}$.

Proof. The first part is evident. The second part is a consequence of the facts that $\mathscr{M}_{d}$ is complete, $\gamma_{\mathscr{Q}}\left(\mathscr{Q}^{s s}\right)=\gamma\left(\mathscr{M}_{d}\right)$, and $\mathscr{Q}^{s s}$ is dense in $\mathscr{Q}^{\mu} \cap \overline{\mathscr{Q}}^{s s}$. q.e.d.

In the sequel, we denote the scheme theoretic image of $\mathscr{M}_{d}$ under $\gamma$ by $\gamma\left(\mathscr{M}_{d}\right)$. We will give a canonical presentation of closed points of $\gamma\left(\mathscr{M}_{d}\right)$ by studying the preimage $\Lambda(s)=\gamma_{\mathscr{Q}}^{-1}(s) \subset \mathscr{Q}^{\mu}$ for any closed point $s \in$ $\gamma\left(\mathscr{M}_{d}\right)$. We first introduce the concept of polystability. A locally free sheaf $E$ is said to be polystable if $E$ splits into a direct sum of $\mu$-stable (with respect to the ample divisor $H$ ) sheaves of the same slopes. A coherent torsion free sheaf $F$ is said to be polystable if the double dual $F^{\vee \vee}$ is polystable. We state the main theorem of this section:

Theorem 4. For any closed $s \in \gamma\left(\mathscr{M}_{d}\right)$, the following assertions are true:

(i) There is a quotient sheaf $F \in \Lambda(s)$ so that $F$ is polystable.

(ii) Let $F_{1}, F_{2} \in \Lambda(s)$ be any two polystable sheaves. Then necessarily $F_{1}^{\vee \vee} \cong F_{2}^{\vee \vee}$ and length $\left(F_{1}^{\vee \vee} / F_{1}\right)_{x}=\operatorname{length}\left(F_{2}^{\vee \vee} / F_{2}\right)_{x}$ for any $x \in X$.

(iii) Let $F_{1}, F_{2} \in \mathscr{Q}^{\mu}$ be any two polystable sheaves. Assume that $F_{1}^{\vee \vee} \cong F_{2}^{\vee \vee}$ and that length $\left(F_{1}^{\vee \vee} / F_{1}\right)_{x}=\operatorname{length}\left(F_{2}^{\vee \vee} / F_{2}\right)_{x}$ for any $x \in X$. Then $\gamma_{\mathscr{Q}}\left(F_{1}\right)=\gamma_{\mathscr{Q}}\left(F_{2}\right)$. 
We divide the proof of the theorem into several lemmas.

Lemma 3.3. For any closed $s \in \gamma\left(\mathscr{M}_{d}\right)$, there is at least one $F \in \Lambda(s)$ that is polystable.

Proof. Let $F \in \Lambda(s)$ be any semistable sheaf. If $F^{\vee \vee}$ is $\mu$-stable, then $F$ is polystable. Otherwise there is a rank-one subsheaf $I \subset F, F / I$ is torsion free, and $\operatorname{deg}(I(-n))=0$. Let $J=F / I$ be the quotient sheaf,

$$
0 \rightarrow I \rightarrow F \rightarrow J \rightarrow 0 .
$$

We claim that $I \oplus J \in \Lambda(s)$. This will establish the lemma since $I \oplus J$ is a polystable sheaf.

Let $\mathbb{C}$ be the set of complex numbers and, as usual, let $p_{1}$ (resp. $p_{2}$ ) be the projection of $X \times \mathbb{C}$ onto the first (resp. second) factor. Let $v_{C} \in$ $\operatorname{Ext}^{1}\left(p_{1}^{*} J, p_{1}^{*} I\right)$ be the extension class, $v_{C}=t \cdot p_{1}^{*} v$, where $v \in \operatorname{Ext}^{1}(J, I)$ is the extension class of (3.3) and $t$ is the uniformizing parameter of $\mathbb{C}$. Let

$$
0 \rightarrow p_{1}^{*} I \rightarrow F_{C} \rightarrow p_{1}^{*} J \rightarrow 0
$$

be the corresponding extension sheaf. $F_{C}$ is a sheaf on $X \times \mathbb{C}$ flat over $\mathbb{C}$. One checks that $F_{C} \otimes k(t)=F$ for generic $t$ and $F_{C} \otimes k(0)=I \oplus J$. To show that $I \oplus J \in \Lambda(s)$, we need to show that $F_{C}$ can in fact be realized as a family of $\oplus^{N} \mathscr{O}$ quotient sheaves. This is obvious from the base change theorem since $h^{i}\left(F_{C} \otimes k(t)\right)=0, i=1,2$, and $h^{0}\left(F_{C} \otimes k(t)\right)=N$ for all $t \in \mathbb{C}$. Here we have used the fact that $I \oplus J$ is $\mu$-semistable and that $n$ is sufficiently large. Since $F_{C} \otimes k(t) \in \mathscr{Q}^{\mu}$ for $t \in \mathbb{C}$, and $F_{C} \otimes k(t) \cong F$ is semistable for $t \neq 0$, it follows that $\gamma_{\mathscr{Q}}\left(F_{C} \otimes k(t)\right)=\gamma(F)=s$ for $t \neq 0$. Then $\gamma_{\mathscr{Q}}\left(F_{C} \otimes k(0)\right)$ is also equal to $s$. Therefore, $\Lambda(s)$ contains at least one polystable sheaf. q.e.d.

To finish the proof of (ii) and (iii) of the theorem, we introduce some subsets of $\mathscr{Q}^{\mu}$. For any locally free sheaf $E$ with $\operatorname{det} E=H^{\otimes 2 n}$ and $c_{2}(E(-n))=d^{\prime} \leq d$, we define

$$
\mathscr{Q}^{\mu}(E)=\left\{F \in \mathscr{Q}^{\mu} \mid F^{\vee \vee} \cong E\right\} .
$$

Let $l: X \rightarrow \mathbb{Z}^{+}$be an integer valued function, where $\mathbb{Z}^{+}$is the set of nonnegative integers. We further define

$$
\mathscr{Q}_{l}^{\mu}(E)=\left\{F \in \mathscr{Q}^{\mu}(E) \mid l_{F}(x) \geq l(x) \text { for any } x \in X\right\},
$$

where $l_{F}$ is the function associated to $F$ via $l_{F}(x)=\operatorname{length}\left(F^{\vee \vee} / F\right)_{x}$.

Lemma 3.4. Let $E$ be any locally free sheaf that is polystable and let $l: X \rightarrow \mathbb{Z}^{+}$be any integer valued function. Assume $c_{2}(E)+\sum_{x} l(x) \leq d$. Then $\mathscr{Q}_{l}^{\mu}(E)$ is a closed, connected algebraic subset of $\mathscr{Q}^{\mu}$. 
We postpone the proof of the lemma to the appendix. Now we finish the proof of Theorem 4 . We first show

Lemma 3.5. Assume $F_{1}, F_{2} \in \mathscr{Q}^{\mu}$ are two polystable sheaves such that $F_{1}^{\vee \vee} \cong F_{2}^{\vee \vee}$ and $l_{F_{1}}(\cdot) \equiv l_{F_{2}}(\cdot)$. Then $\gamma_{\mathscr{Q}}\left(F_{1}\right)=\gamma_{\mathscr{Q}}\left(F_{2}\right)$.

Proof. Let $E=F_{1}^{\vee \vee}$ and let $l: X \rightarrow \mathbb{Z}^{+}$be the function $l(\cdot)=l_{F_{1}}(\cdot)$. Then $F_{1}, F_{2} \in \mathscr{Q}_{l}^{\mu}(E)$. Since $\mathscr{Q}_{l}^{\mu}(E)$ is connected, the lemma is proved if we can show that $\gamma_{\mathscr{Q}}(g(C))$ is a single point for any smooth irreducible curve $C$ and $g: C \rightarrow \mathscr{Q}_{l}^{\mu}(E)$.

Let $C$ be an affine curve, let $g: C \rightarrow Q_{l}^{\mu}(E)$ be any morphism, and let $F_{C}$ be the pullback sheaf of the universal quotient sheaf on $\mathscr{Q}^{\mu} . F_{C}^{\vee \vee}$ is isomorphic to $p_{1}^{*} E$. Then $F_{C}$ belongs to the exact sequence

$$
0 \rightarrow F_{C} \rightarrow p_{1}^{*} E \rightarrow A_{C} \rightarrow 0 .
$$

The surjection $p_{1}^{*} E \rightarrow A_{C}$ induces a morphism $\bar{g}: C \rightarrow \operatorname{Quot}_{E}^{d-d^{\prime}}(l)$, where $\operatorname{Quot}_{E}^{d-d^{\prime}}(l)$ stands for the Grothendieck's Quot-scheme parametrizing all quotient sheaves $A$ of $E$ with length $(A)=d-d^{\prime}$ and length $\left(A_{x}\right) \geq$ $l(x)$ for any $x \in X$. Since $\operatorname{Quot}_{E}^{d-d^{\prime}}(l)$ is complete, there is a smooth completion $\bar{C}$ of $C$ and $h: \bar{C} \rightarrow \operatorname{Quot}_{E}^{d-d^{\prime}}(l)$ extending the morphism $\bar{g}: C \rightarrow \operatorname{Quot}_{E}^{d-d^{\prime}}(l)$. Let $F_{\bar{C}}$ be the kernel of $p_{1}^{*} E \rightarrow A_{\bar{C}} \rightarrow 0$, where $A_{\bar{C}}$ is the pullback of the universal quotient sheaf on $\operatorname{Quot}_{E}^{d-d^{\prime}}(l)$ via $h$. By Proposition 2.3 there is a line bundle $\mathscr{L}_{k}$ on $\bar{C}$, and for any smooth $D \in|k H|$ and $\theta_{D} \in \mathrm{Jac}^{g(D)-1}(D)$ we have

$$
\mathscr{L}_{k}=\operatorname{Det}\left(R p_{2, *} F_{\bar{C}}(-n)_{\mid D} \otimes p_{1}^{*} \theta_{D}\right)^{-1} .
$$

In particular, if $D$ is the divisor away from the support of $l(\cdot)$ on $X$, then

$$
\begin{aligned}
\mathscr{L}_{k} & \cong \operatorname{Det}\left(R p_{2, *} F_{\bar{C}}(-n)_{\mid D} \otimes p_{1}^{*} \theta_{D}\right)^{-1} \\
& =\operatorname{Det}\left(R p_{2, *} p_{1}^{*} E_{\mid D} \otimes p_{1}^{*} \theta_{D}\right)^{-1}=\mathscr{O}_{\bar{C}} .
\end{aligned}
$$

Now assume $D \in|k H|$ is any smooth divisor. Since $g(C) \subset \mathscr{Q}^{\mu}$, there is a canonical isomorphism (up to scalar) $g^{*} \mathscr{L}_{\mathscr{Q}}\left(D, \theta_{D}\right)=\mathscr{L}_{k \mid C}$. Let $g^{*}: \mathscr{V}_{k}^{\mathscr{Q}}(m) \rightarrow H^{0}\left(C, \mathscr{L}_{k \mid C}^{\otimes m}\right)$ be the induced homomorphism from Proposition 2.5 between the sets of global sections of the corresponding line bundles. Since the determinant line bundles and the isomorphism $g^{*}$ are canonical, and since $\mathscr{V}_{k}^{\mathscr{Q}}(m)$ are spanned by $\mathscr{V}_{D}^{\mathscr{Q}}(m)$, by Proposition 2.5 , any section in $g^{*}\left(\mathscr{V}_{k}^{\mathscr{Q}}(m)\right) \subset H^{0}\left(C, \mathscr{L}_{k \mid C}^{\otimes m}\right)$ extends to a section in 
$H^{0}\left(\bar{C}, \mathscr{L}_{k}^{\otimes m}\right)$. Finally, since $\mathscr{L}_{k} \cong \mathscr{O}_{\bar{C}}$, it follows that $H^{0}\left(\bar{C}, \mathscr{L}_{k}^{\otimes m}\right) \cong$ $\mathbb{C}$. So the image $\gamma_{\mathscr{Q}}(g(C)) \subset \mathbf{P}\left(\mathscr{V}_{k}^{\mathscr{Q}}(m)^{\vee}\right)$ must be a single point. Thus we have established part (iii) of the theorem.

Lemma 3.6. Let $s_{1}, s_{2} \in \mathscr{Q}^{\mu}$ be two closed points and let $F_{1}, F_{2}$ be the corresponding quotient sheaves such that both are polystable. Assume $F_{1}^{\vee \vee} \neq F_{2}^{\vee \vee}$, or $F_{1}^{\vee \vee}=F_{2}^{\vee \vee}$ and $l_{F_{1}}(\cdot) \not \equiv l_{F_{2}}(\cdot)$. Then $\gamma_{\mathscr{Q}}\left(F_{1}\right) \neq \gamma_{\mathscr{Q}}\left(F_{2}\right)$.

Proof. Assume $F_{1}^{\vee \vee} \neq F_{2}^{\vee \vee}$. Then, according to our choice of $k$, $F_{1 \mid D} \neq F_{2 \mid D}$ for generic $D \in|k H|$. Since $\mathscr{M}(D) \rightarrow \mathbf{P}\left(V_{D}(m)^{\vee}\right)$ is an immersion, there is a section $v \in V_{D}(m)$, where $v$ vanishes at $F_{1 \mid D}$ and is nonzero at $F_{2 \mid D}$. Therefore, for the pullback section $\tilde{v} \in \mathscr{V}_{k}^{\mathscr{Q}}(m), \tilde{v}\left(F_{1}\right)=$ 0 while $\tilde{v}\left(F_{2}\right) \neq 0$. Hence, $\gamma_{\mathscr{Q}}\left(F_{1}\right) \neq \gamma_{\mathscr{Q}}\left(F_{2}\right)$.

For the second case, let $E=F_{1}^{\vee \vee}$ and let $\bar{x} \in X$ be the point such that $l_{F_{1}}(\bar{x})>l_{F_{2}}(\bar{x}) \geq 0$. We first choose a smooth $D_{0} \in|k H|$ such that $\bar{x} \in D_{0}$ and $F_{1 \mid D_{0}}$ and $F_{2 \mid D_{0}}$ are locally free at $D_{0} \mid \bar{x}$. Let $D_{t}$ be a linear series in $|k H|$ containing $D_{0}$ such that $\bar{x}$ is not its base point. Following the proof of Lemma 2.2, we can form a smooth surface $q: Y \rightarrow X$ by blowing up $X$ along the base points of the linear series. Let $\theta$ be the line bundle on $Y$ such that $\operatorname{deg} \theta_{\mid D_{0}}=g\left(D_{0}\right)-1$. Let $\pi: Y \rightarrow \mathbf{P}^{1}$ be the projection. We then form the determinant line bundles

$$
\mathscr{L}_{i, \mathbf{P}^{1}}=\operatorname{Det}\left(R \pi_{*}\left(q^{*} F_{i} \otimes \theta\right)\right)^{-1}
$$

on $\mathbf{P}^{1}$ with $i=1,2$. By Lemma $2.2, \mathscr{L}_{1, \mathbf{P}^{1}} \cong \mathscr{L}_{2, \mathbf{P}^{1}} \cong \mathscr{O}(\alpha)$. Now let $C \subset \mathbf{P}^{1}$ be the open neighborhood of 0 such that for any $t \in C, \pi^{-1}(t)$ is smooth, and for $0 \neq t \in C, F_{1}$ and $F_{2}$ are locally free at $\pi^{-1}(t)$. We think of $\pi^{-1}(C) \subset Y$ as a family of smooth curves parametrized by $C$. We denote this family by $\pi: D_{C} \rightarrow C$.

Let $\psi: \mathscr{M}\left(D_{C} / C\right) \rightarrow C$ be the flat family of projective varieties with $q^{-1}(t)=\mathscr{M}\left(D_{t}\right)$. Since $D_{C} \rightarrow C$ is a flat family of smooth curves, such an $\mathscr{M}\left(D_{C} / C\right)$ does exist [15]. Moreover, by using a method similar to that in $\S 2$, we can show that there is a line bundle $\mathscr{L}_{D_{C}}$ on $\mathscr{M}\left(D_{C} / C\right)$, $\mathscr{L}_{D_{C}} \otimes k(t)=\mathscr{L}_{D_{t}}$. By shrinking $C$ if necessary, we can assume that for large $m$ there is a $v \in H^{0}\left(\mathscr{M}\left(D_{C} / C\right), \mathscr{L}_{D_{C}}^{\otimes m}\right), v_{\mid \mathscr{M}\left(D_{t}\right)} \in V_{D_{t}}(m)$, so that $\left.v\right|_{\mathscr{K}\left(D_{0}\right)}$ is nonvanishing at $E_{\mid D_{0}}$. Since $F_{i}, i=1,2$, are locally free at the base points of the family $D_{t}$, we have the following exact sequences on $D_{C}$ :

$$
0 \rightarrow q^{*} F_{i} \rightarrow q^{*} E \rightarrow A_{i, C} \rightarrow 0
$$


where $q: D_{C} \rightarrow X$ is the obvious map, and $A_{i, C}$ are torsion sheaves on $D_{C}$.

In the following, we let the indices $i$ be either one or two. Let $w_{i}$ be the closed points of $\mathscr{Q}^{\mu}$ representing the quotient sheaves $F_{i}$. If we fix a trivialization of $\mathscr{O}(\alpha)_{\mid C}$ and use Lemma 2.2, then we have the following canonical isomorphisms of line bundles on $C$ :

$$
\varphi_{i, C}:\left.\operatorname{Det}\left(R \pi_{*} q^{*} F_{i}(-n) \otimes \theta\right)^{-1} \cong \mathscr{L}_{\mathscr{Q}, k}\right|_{w_{i}} \otimes_{k} \mathscr{O}_{C} .
$$

By Proposition 2.5, for any $t \in C$, there is a canonical section $\tilde{v}_{i} \in$ $H^{0}\left(\mathscr{Q}^{\mu}, \mathscr{L}_{\mathscr{Q}, k}^{\otimes m}\right)$ which is provided by the rational map $\mathscr{Q}^{\mu} \rightarrow \mathscr{M}\left(D_{t}\right)$ and $v_{t} \in V_{D_{t}(m)}$, where $v_{t}$ is the restriction of $v \in H^{0}\left(\mathscr{M}\left(D_{C} / C\right), \mathscr{L}_{D_{C}}^{\otimes m}\right)$ to $\mathscr{M}\left(D_{t}\right)$. Note that this construction is canonical. Thus $\tilde{v}_{t}$ with $t \in C$ can be viewed as a section $\tilde{v}_{C} \in H^{0}\left(\mathscr{Q}^{\mu} \times C, g^{*} \mathscr{L}_{\mathscr{Q}, k}^{\otimes m}\right)$, where $g: \mathscr{Q}^{\mu} \times C \rightarrow$ $\mathscr{Q}^{\mu}$. We denote the restriction of $\tilde{v}_{C}$ to $\left\{w_{i}\right\} \times C \subset \mathscr{Q}^{\mu} \times C$ by $\tilde{v}_{C}^{i} \in$ $H^{0}\left(\left\{w_{i}\right\} \times C,\left.g^{*} \mathscr{L}_{\mathscr{Q}, k}^{\otimes m}\right|_{w_{i} \times C}\right)$. Now using (3.7), we have isomorphisms

$$
\phi_{i}: \operatorname{Det}\left(R \pi_{*}\left(q^{*} F_{i}(-n) \otimes \theta\right)\right)^{-1} \cong \operatorname{Det}\left(R \pi_{*}\left(q^{*} E(-n) \otimes \theta\right)\right)^{-1}\left(l_{i} 0\right),
$$
where $l_{i}=$ length $\left(A_{i, 0}\right)=\operatorname{length}\left(E / F_{i}\right)_{\bar{x}}$. Since $\varphi_{1, C}$ and $\varphi_{2, C}$ are canonical, and $F_{1 \mid \pi^{-1}(C \backslash 0)}=F_{2 \mid \pi^{-1}(C \backslash 0)}$, we have

$$
\phi_{1}\left(\varphi_{1, C}^{*}\left(\tilde{v}_{C \mid C \backslash 0}^{1}\right)\right)=\phi_{2}\left(\varphi_{2, C}^{*}\left(\tilde{v}_{C \mid C \backslash 0}^{2}\right)\right)
$$

as sections of the line bundle $\operatorname{Det}\left(R \pi_{*} q^{*} E(-n) \otimes \theta\right)^{-1}$ over $C \backslash 0$. Since we assumed that $v_{0}\left(E_{\mid D_{0}}\right) \neq 0, \phi_{1}\left(\varphi_{1, C}^{*}\left(\tilde{v}_{C|C| 0}^{1}\right)\right)$ is nontrivial. Now assume that the extension of $\phi_{1}\left(\varphi_{1, C}^{*}\left(\tilde{v}_{C \mid C \backslash 0}^{1}\right)\right)$ in $\operatorname{Det}\left(R \pi_{*} q^{*} E(-n) \otimes \theta\right)^{-1}$ over $C$ has vanishing order $l$ at $0 \in C$. Then $\tilde{v}_{C}^{1}$ (resp. $\tilde{v}_{C}^{2}$ ) will have vanishing order $l+l_{1}$ (resp. $l+l_{2}$ ) at 0 by (3.8). Since we assumed that $l_{1} \neq l_{2}$, then for generic $t_{1}, t_{2} \in C$,

$$
\tilde{v}_{C}^{1}\left(t_{1}\right) / \tilde{v}_{C}^{1}\left(t_{2}\right) \neq \tilde{v}_{C}^{2}\left(t_{1}\right) / \hat{v}_{C}^{2}\left(t_{2}\right) \text {. }
$$

Therefore, $\gamma_{\mathscr{Q}}\left(w_{1}\right) \neq \gamma_{\mathscr{Q}}\left(w_{2}\right)$. This finishes the proof of the theorem. q.e.d.

By Theorem 4, every closed point $s \in \gamma\left(\mathscr{M}_{d}\right)$ is represented by a unique pair $(E, l)$, where $E$ is a polystable vector bundle and $l: X \rightarrow \mathbb{Z}^{+}$is an integer function such that $c_{2}(E)+\sum_{x \in X} l(x)=d$. For $d>0$, if we denote the open subset of $\mathscr{M}_{d}$ consisting of $\mu$-stable locally free sheaves by $\mathscr{M}_{d}^{\mu}$, then a corollary of the theorem states that

$$
\gamma: \mathscr{M}_{d}^{\mu} \rightarrow \gamma\left(\mathscr{M}_{d}^{\mu}\right) \subset \gamma\left(\mathscr{M}_{d}\right)
$$


is one-to-one set-theoretical. In fact, more is true.

Proposition 3.7. For any $d>0$ and sufficiently large $k$ and $m \gg 0$, the morphism $\gamma: \mathscr{M}_{d}^{\mu} \rightarrow \gamma\left(\mathscr{M}_{d}^{\mu}\right) \subset \gamma\left(\mathscr{M}_{d}\right)$ is an isomorphism.

Proof. We only need to show that $\gamma$ is unramified at each point $s \in$ $\mathscr{M}_{d}^{\mu}$. Indeed, for $k$ large enough, $D \in|k H|$ smooth, and $E, F \in \mathscr{M}_{d}^{\mu}$, we have $\operatorname{Hom}(E, F) \rightarrow \operatorname{Hom}\left(E_{\mid D}, F_{\mid D}\right)$ is surjective and $\operatorname{Ext}_{X}^{1}(E, E) \rightarrow$ $\operatorname{Ext}_{D}^{1}\left(E_{\mid D}, E_{\mid D}\right)$ is injective. It is straightforward to check that $\mathscr{M}_{d}^{\mu} \rightarrow$ $\mathscr{M}(D)$ is an immersion. When $m \gg 0, \mathscr{M}(D) \rightarrow \mathbf{P}\left(V_{D}(m)^{\vee}\right)$ is also an immersion. Thus $\mathscr{M}_{d}^{\mu} \rightarrow \mathbf{P}\left(\mathscr{V}_{D}^{\mathscr{M}}(m)^{\vee}\right)$ is an immersion, and $\gamma: \mathscr{M}_{d}^{\mu} \rightarrow$ $\gamma\left(\mathscr{M}_{d}^{\mu}\right) \subset \mathbf{P}\left(\mathscr{V}_{k}^{\mathscr{M}}(m)^{\vee}\right)$ is an isomorphism. We leave the details to the reader.

\section{Uhlenbeck's compactification of the moduli space $\mathscr{M}_{d}^{\mu}$}

In this section, we investigate the relations between the space of gauge equivalent classes of ASD connections and the image scheme $\gamma\left(\mathscr{M}_{d}\right)$. Let us first recall the theory of anti-self-dual connections. Let $X$ be an oriented four-manifold, let $\mathbf{g}$ be a Riemannian metric on $X$, and let $E$ be a rank two complex vector bundle over $X$ with $c_{1}(E)=0$ and $c_{2}(E)=d$. By an $\mathrm{SU}(2)$ structure on $E$ we mean a smooth isomorphism $\wedge^{2} E \cong \mathbb{C} \times X$ coupled with a Hermitian metric $h$ on $E$ so that $\operatorname{det} h=1$. By an $\mathrm{SU}(2)$ isomorphism (resp. gauge transformation) of two $\mathrm{SU}(2)$ vector bundles (resp. an $\mathrm{SU}(2)$ vector bundle) we mean a smooth isomorphism (resp. an automorphism) preserving the $\mathrm{SU}(2)$ structures. By abuse of notation, we will use $(E, h)$ to denote the vector bundle $E$ with the prescribed $S U(2)$ structure. A connection $D$ is said to be an $\mathrm{SU}(2)$ connection if it is a linear connection on $E$ preserving $h . D$ is called anti-self-dual if in addition $D$ satisfies

$$
*_{\mathbf{g}} F(D)+F(D)=0 \text {, }
$$

where $*_{\mathbf{g}}$ is the star operator of $\mathbf{g}$ and $F(D)$ is the curvature tensor of the connection $D$. Equivalently, if we denote by $P_{+}: \bigwedge^{2} T^{*} X \rightarrow \bigwedge_{+}^{2} T^{*} X$ the projection sending any two-form to its self-dual part, then $P_{+}(F(D))=0$. Two connections $D_{1}$ and $D_{2}$ on $(E, h)$ are said to be gauge equivalent if there is a gauge transformation $g$ on $(E, h)$ such that $g^{*}\left(D_{2}\right)=D_{1}$, where $g^{*}\left(D_{2}\right)=g^{-1} \circ G_{2} \circ g$.

We denote by $\mathscr{N}_{d}(\mathbf{g})$ the space of gauge equivalent classes of irreducible ASD connections on $(E, h)$. We will abbreviate $\mathscr{N}_{d}(\mathbf{g})$ to $\mathscr{N}_{d}$ when the 
Riemannian metric $\mathbf{g}$ is clear from the context. $\mathscr{N}_{d}$ is nonempty if $d$ is large [20] and $\mathscr{N}_{d}$ is smooth at $D$ is $H_{[D]}^{2}\left(X, \operatorname{Ad} E_{h}\right)=\{0\}$. Here $H_{[D]}^{i}\left(X, \operatorname{Ad} E_{h}\right)$ are cohomology groups of the elliptic complex

$$
\Omega^{0}\left(\operatorname{Ad} E_{h}\right) \stackrel{D}{\longrightarrow} \Omega^{1}\left(\operatorname{Ad} E_{h}\right) \stackrel{D_{+}}{\longrightarrow} \Omega_{+}^{2}\left(\operatorname{Ad} E_{h}\right)
$$

with $D_{+}=P_{+} D$, where $\mathrm{Ad} E_{h}$ is the adjoint bundle of $(E, h)$. (For details, see [5].) Further, we denote by $\widetilde{\mathscr{N}_{d}}$ the space of gauge equivalent classes of ASD connections (not necessarily irreducible) on $(E, h)$. If $X$ is an algebraic surface and $\mathbf{g}$ is the Hodge metric of the ample line bundle $H$, then Donaldson proved that $D$ is an ASD connection if and only if the induced $\bar{\partial}_{D}$ operator defines a $\mu$-polystable holomorphic structure on $E$. More precisely, we have

Proposition 4.1 (Donaldson). Let $d>0$. Let $\mathscr{N}_{d}$ be the space of gauge equivalent classes of irreducible $A S D$ connections on $E$ and let $\mathscr{M}_{d}^{\mu} \subset \mathscr{M}_{d}$ be the open subset consisting of locally free $\mu$-stable sheaves. Then there is a canonical homeomorphism $\sigma: \mathscr{M}_{d}^{\mu} \rightarrow \mathscr{N}_{d}$. Further, $\sigma$ is a diffeomorphism at $s \in \mathscr{M}_{d}^{\mu}$ if $\mathscr{M}_{d}^{\mu}$ is smooth at $s$ or equivalently $\mathscr{N}_{d}$ is smooth at $\sigma(s)$.

Proof. Assume $(E, \bar{\partial})$ is a $\mu$-stable vector bundle. By [1], there is a unique (up to gauge equivalence) Hermitian-Einstein connection $D$ such that $\bar{\partial}_{D}=\bar{\partial} . D$ is automatically anti-self-dual. We define $\sigma((E, \bar{\partial}))=$ $D$. That the map $\sigma$ is a homeomorphism is guaranteed by the uniqueness of the solutions of the elliptic system which solves the Hermitian-Einstein connections on $(E, \bar{\partial})$.

For diffeomorphism, one observes that $s=(E, \partial)$ (resp. $\sigma(s))$ is a smooth point of $\mathscr{M}_{d}^{\mu}$ (resp. $\mathscr{N}_{d}$ ) if $H^{2}\left(X, \mathscr{E}_{n d^{0}}(E)\right.$ ) (resp. $H_{[\sigma(s)]}^{2}(X$, Ad $\left.\left.E_{h}\right)\right)$ vanishes. On the other hand, $H^{2}\left(X, \mathscr{E}_{n} d^{0}(E)\right) \cong H_{[\sigma(s)]}^{2}(X$, Ad $E_{h}$ ) (see [11, Proposition 2.4]). Therefore, $s \in \mathscr{M}_{d}^{\mu}$ is smooth if and only if $\sigma(s) \in \mathscr{N}_{d}$ is smooth. To finish the proof, it suffices to show that the homomorphism $\sigma_{*}$,

$$
\begin{array}{ccc}
T_{s} \mathscr{M}_{d}^{\mu} & \stackrel{\sigma_{*}}{\longrightarrow} & T_{\sigma(s)} \mathscr{N}_{d} \\
\| \iota & & \| l \\
H^{1}\left(X, \mathscr{E} n d^{0}(E)\right) & & H_{[\sigma(s)]}^{1}\left(X, \mathscr{E} n d^{0}(E)\right),
\end{array}
$$

is an isomorphism. This is apparent from [11]. q.e.d.

$\mathscr{N}_{d}$ is not compact since $\mathscr{M}_{d}^{\mu}$ is not compact. There is a natural compactification of $\mathscr{N}_{d}$ by using Uhlenbeck's weak compactness theorem on ASD connections. 
Proposition 4.2 (Uhlenbeck's weak compactness theorem [5]). Let $A_{i}$ be a sequence of ASD connections on $(E, h)$. By passing to a subsequence $\left\{i^{\prime}\right\} \subset\{i\}$, there exist

(a) a finite set of points $x_{1}, x_{2}, \cdots, x_{p}$ with multiplicities $m_{1}, \cdots$, $m_{p}$, and

(b) an ASD connection $A_{0}$ on an $\mathrm{SU}(2)$ vector bundle $\left(E_{0}, h_{0}\right)$, such that

(1) there are gauge transformations $g_{i^{\prime}}$ of $(E, h)$ and an $\mathrm{SU}(2)$ isomorphism $g_{\infty}: E_{\mid X \backslash \cup x_{i}} \rightarrow E_{0 \mid X \backslash \cup x_{i}}$ such that for any compact subset $K \subset$ $\left(X \backslash \bigcup x_{i}\right)$,

$$
\lim _{i^{\prime} \rightarrow \infty} g_{i^{\prime}}^{*}\left(A_{i^{\prime}}\right)_{\mid K}=g_{\infty}^{*}\left(A_{0}\right)_{\mid K} \quad \text { in the } C^{1} \text { topology; }
$$

(2) considering $\left|F_{A^{\prime}}\right|^{2}$ and $\left|F_{A_{0}}\right|^{2}$ as measures on $X$ and using the weak ${ }^{*}$-topology on the space of these measures, we have

$$
\lim _{i^{\prime} \rightarrow \infty}\left|F_{A_{i}}\right|^{2}=\left|F_{A_{0}}\right|^{2}+8 \pi^{2} \sum_{i=1}^{p} m_{i} \delta_{x_{i}},
$$

where $\delta_{x}$ is the singular measure of total mass 1 supported at $x$.

According to Uhlenbeck's weak compactness theorem, for any sequence of ASD connections, say $\left\{A_{i}\right\}$, there is a subsequence $\left\{A_{i^{\prime}}\right\} \subset\left\{A_{i}\right\}$ such that their gauge equivalence classes converge weakly to the pair $\left(A_{0}, \delta\right)$, where $A_{0} \in \mathscr{N}_{d_{0}}$ and $\delta$ is a singular measure of total mass $d-d_{0}$. Uhlenbeck's removable singularity theorem of ASD connections shows such a pair is unique (depending on the subsequence $\left\{A_{i^{\prime}}\right\}$ ). We denote by $S^{l} X$ the $l$ th-symmetric power of $X$. To any point $s \in S^{l} X$, we associate a singular measure $\delta_{s}$ of total mass $l . \delta_{s}$ has mass $c$ at the point $x$ if $x$ has multiplicity $c$ in $s$. Thus $\delta$ can be thought of as a point in $S^{d-d_{0}} X$. Now if we view $\left(A_{0}, \delta\right) \in \widetilde{\mathcal{N}_{d_{0}}} \times S^{d-d_{0}} X$ as the weak limit of the sequence $\left\{A_{i^{\prime}}\right\} \subset \mathscr{N}_{d}$, then there is a compactification $\bar{N}_{d}$ of $\mathscr{N}_{d}$,

$$
\overline{\mathscr{N}}_{d} \subset \coprod_{j=0}^{d} \widetilde{\mathcal{N}_{j}} \times S^{d-j} X .
$$

$\overline{\mathscr{N}}_{d}$ is called Uhlenbeck's compactification of the space of gauge equivalent classes of irreducible ASD connections [2, §3].

It should be noted that both $\mathscr{M}_{d}$ and $\bar{N}_{d}$ are compactifications of the space $\mathscr{M}_{d}^{\mu} \cong \mathscr{N}_{d}$. The former is constructed by using algebraic geometry while the latter is defined for any smooth Riemannian four-manifold. They 
are drastically different in nature. For instance, the subvariety $\mathscr{M}_{d} \backslash \mathscr{M}_{d}^{\mu}$ is a subset of complex codimension 1 , while $\overline{\mathscr{N}}_{d} \backslash \mathcal{N}_{d}$ is of real codimension four in general.

In $\S 3$, we introduced another space $\gamma\left(\mathscr{M}_{d}\right)$ containing $\gamma\left(\mathscr{M}_{d}^{\mu}\right)$ as a dense subset. Hence $\gamma\left(\mathscr{M}_{d}\right)$ can be thought of as a compactification of $\mathscr{M}_{d}^{\mu}$ as well. To be more precise, we let $\overline{\mathscr{M}}_{d}^{\mu}$ be the closure of $\mathscr{M}_{d}^{\mu}$ in $\mathscr{M}_{d}$ with the reduced scheme structure and let $\gamma\left(\overline{\mathscr{M}}_{d}^{\mu}\right) \subset \gamma\left(\mathscr{M}_{d}\right)$ be the image scheme. Note that for $d$ large enough, $\mathscr{M}_{d}$ is normal [13] and thus $\overline{\mathscr{M}}_{d}^{\mu}=\mathscr{M}_{d}$ and $\gamma\left(\overline{\mathscr{M}}_{d}^{\mu}\right)=\gamma\left(\mathscr{M}_{d}\right)$. The main theorem of this section is the following.

Theorem 5. Let $X$ be any algebraic surface and let $\mathbf{g}$ be the Hodge metric of the ample divisor $H$. For any $d>0$ where $\mathscr{M}_{d}^{\mu} \neq \varnothing$, there is a homeomorphism

$$
\bar{\sigma}: \gamma\left(\overline{\mathscr{M}}_{d}^{\mu}\right) \rightarrow \overline{\mathscr{N}}_{d}
$$

such that $\bar{\sigma}=\sigma$ when restricted to $\gamma\left(\mathscr{M}_{d}^{\mu}\right) \subset \gamma\left(\overline{\mathscr{M}}_{d}^{\mu}\right)$.

Corollary 4.3. With the notation as above, there is a complex structure on $\overline{\mathscr{N}}_{d}$ making it a reduced projective scheme. Furthermore, there is a morphism $\gamma: \overline{\mathscr{M}}_{d}^{\mu} \rightarrow \overline{\mathscr{N}}_{d}$ from Gieseker's compactification of $\mathscr{N}_{d}$ to Uhlenbeck's compactification of $\mathscr{N}_{d}$.

The main effort of this section is devoted to the proof of Theorem 5. In the following, we shall view $\gamma\left(\overline{\mathscr{M}}_{d}^{\mu}\right)$ and other reduced schemes as analytic varieties and endow them with usual topology. We will prove Theorem 5 by showing

Proposition 4.4. Let $\left\{F_{i}\right\} \subset \gamma\left(\mathscr{M}_{d}^{\mu}\right)$ be any sequence of $\mu$-stable vector bundles. Assume that $\left\{F_{i}\right\}$ converges to $\left(F_{0}, l\right)$ in $\gamma\left(\overline{\mathscr{M}}_{d}^{\mu}\right)$ and that $\left\{\sigma\left(F_{i}\right)\right\}$ converges to $\left(E_{0}, A_{0}, \delta\right) \in \overline{\mathscr{N}}_{d}$. Then $F_{0} \cong\left(E_{0}, \bar{\partial}_{A_{0}}\right)$ and $l \equiv \delta$ under the obvious identification between $l: X \rightarrow \mathbb{Z}^{+}$and elements of $S^{c} X$, where $c=\sum_{x \in X} l(x)$.

Proof of Theorem 5 (assuming Proposition 4.4). By Theorem 4, each closed point $s \in \gamma\left(\overline{\mathscr{M}}_{d}^{\mu}\right)$ is represented by the pair $(E, l)$, where $E$ is a polystable vector bundle and $l(\cdot)$ is an integer valued function with $c_{2}(E)+\sum_{x} l(x)=d$. Such an $E$ admits a unique (up to gauge equivalence) Hermitian-Einstein (or equivalently ASD) connection $A$. Hence $(E, l)$ associates uniquely to a point

$$
(E, A, \delta) \in \coprod_{j=0}^{d} \widetilde{\mathcal{N}_{j}} \times S^{d-j} X,
$$


where $\delta=\left\{m_{i} \delta_{x_{i}}\right\}$ with $m_{i}=l\left(x_{i}\right)$. We denote such an identification by $\bar{\sigma}$ :

$$
\bar{\sigma}: \gamma\left(\overline{\mathscr{M}}_{d}^{\mu}\right) \rightarrow \coprod_{j=0}^{d} \widetilde{\mathscr{N}_{j}} \times S^{d-j} X .
$$

Clearly, $\bar{\sigma}$ is a one-to-one map which coincides with $\sigma$ when restricted to the open dense subset $\gamma\left(\mathscr{M}_{d}^{\mu}\right)$. Next we show that $\bar{\sigma}$ is continuous. It suffices to show that whenever $\left\{s_{i}\right\} \subset \gamma\left(\overline{\mathscr{M}}_{d}^{\mu}\right)$ is a sequence which converges to $s_{0}$ in $\gamma\left(\overline{\mathscr{M}}_{d}^{\mu}\right)$, then $\bar{\sigma}\left(s_{i}\right)$ converges to $\bar{\sigma}\left(s_{0}\right)$. Since both spaces $\gamma\left(\overline{\mathscr{M}}_{d}^{\mu}\right)$ and $\overline{\mathscr{N}}_{d}$ are compact, and $\gamma\left(\mathscr{M}_{d}^{\mu}\right) \subset \gamma\left(\overline{\mathscr{M}}_{d}^{\mu}\right)$ and $\sigma\left(\gamma\left(\mathscr{M}_{d}^{\mu}\right)\right)=\mathscr{N}_{d} \subset$ $\overline{\mathscr{N}}_{d}$ are dense, an easy argument shows that all we have to show is that whenever $\left\{s_{i}\right\}$ is a sequence in $\gamma\left(\mathscr{M}_{d}^{\mu}\right)$ with $\lim s_{i}=s_{0} \in \gamma\left(\overline{\mathscr{M}}_{d}^{\mu}\right)$ and $\lim \sigma\left(s_{i}\right)=t_{0} \in \overline{\mathscr{N}}_{d}$, then $\bar{\sigma}\left(s_{0}\right)=t_{0}$. That is exactly what we show in Proposition 4.4. Finally, $\bar{\sigma}\left(\gamma\left(\overline{\mathscr{M}}_{d}^{\mu}\right)\right) \subset \overline{\mathscr{N}}_{d}$ is a dense closed subset, so $\bar{\sigma}\left(\gamma\left(\overline{\mathscr{M}}_{d}^{\mu}\right)\right)=\overline{\mathscr{N}}_{d}$. Thus $\bar{\sigma}$ is one-to-one, onto, and continuous, and hence a homeomorphism. q.e.d.

Let $\left\{F_{i}\right\}$ be a sequence of $\mu$-stable vector bundles in $\mathscr{M}_{d}^{\mu}$. By Proposition 4.1, there are Hermitian metrics $h_{i}$ on $F_{i}$ so that the induced connections are Hermitian-Einstein. Let $(E, h)$ be the $\mathrm{SU}(2)$ vector bundle on $X$ having the same topological type as $F_{i}$. There are ASD connections $A_{i}$ on $(E, h)$ and smooth isomorphisms $\tilde{\varphi}_{i}:\left(F_{i}, h_{i}\right) \rightarrow(E, h)$ such that $\tilde{\varphi}_{i}^{*}\left(A_{i}\right)$ are the corresponding Hermitian-Einstein connections on $\left(F_{i}, h_{i}\right)$. Thanks to Uhlenbeck's weak compactness theorem, there are $\mathrm{SU}(2)$ gauge transformations $g_{i}$ on $(E, h)$ such that a subsequence of $g_{i}^{*}\left(A_{i}\right)$ converges in the weak sense. Let $\varphi_{i}=g_{i}^{-1} \circ \tilde{\varphi}_{i}$.

Lemma 4.5. For any sequence $\left\{F_{i}\right\}$ of $\mu$-stable vector bundles in $\mathscr{M}_{d}^{\mu}$ and the corresponding Hermitian-Einstein metrics $h_{i}$ on $F_{i}$, there is a sequence of $A S D$ connections $A_{i}$ on $(E, h)$ and smooth isomorphisms $\varphi_{i}:\left(F_{i}, h_{i}\right) \rightarrow(E, h)$ such that for each $i, \varphi_{i}^{*}\left(A_{i}\right)$ is the HermitianEinstein connection of $\left(F_{i}, h_{i}\right)$. Moreover, there is an $\mathrm{SU}(2)$ vector bundle $\left(E_{0}, h_{0}\right)$, a finite subset $\Lambda=\left\{x_{1}, x_{2}, \cdots, x_{p}\right\} \subset X$, and an isomorphism

$$
\varphi:(E, h)_{\mid X \backslash \Lambda} \rightarrow\left(E_{0}, h_{0}\right)_{\mid X \backslash \Lambda}
$$

such that by passing to a subsequence $\left\{i^{\prime}\right\} \subset\{i\}$,

$$
\lim _{i^{\prime} \rightarrow \infty} A_{i^{\prime}}=\varphi^{*} A_{0} \quad \text { weakly on } X \backslash \Lambda \text {, }
$$

where $A_{0}$ is an ASD connection on $\left(E_{0}, h_{0}\right)$, and there is a singular measure $\delta$ such that (4.3) holds.

We fix such a subsequence given by the lemma and still denote it by $F_{i}$ (and $A_{i}$ ). We choose $n$ large enough so that whenever $F$ is a 
$\mu$-semistable sheaf with $\operatorname{det} F=\mathscr{O}$ and $c_{2}(F) \leq d$, then $h^{i}\left(H^{\otimes n} \otimes F\right)=0$ for $i \geq 1$ and $H^{0}\left(H^{\otimes n} \otimes F\right)$ generates $H^{\otimes n} \otimes F$. Thus $N=h^{0}\left(H^{\otimes n} \otimes F_{i}\right)$ is independent of $i$. We fix the Hermitian metric on $H$ whose Chern form is the Kähler class. Let $H^{\otimes n} \otimes E$ has the induced metric. Let $e_{i}^{\alpha}$, $\alpha=1, \cdots, N$ be sections of $H^{\otimes n} \otimes E$ such that $e_{i}^{\alpha}$ are holomorphic under the holomorphic structure $\bar{\partial}_{A_{i}}$ and such that for all $\alpha, \beta$,

$$
\int_{X}\left\langle e_{i}^{\alpha}, e_{i}^{\beta}\right\rangle_{h} * 1=\delta_{\alpha \beta}
$$

Lemma 4.6. With the notation as before, there is a subsequence $\left\{i^{\prime}\right\} \subset$ \{i\} such that for any $1 \leq \alpha \leq N,\left\{e_{i^{\prime}}^{\alpha}\right\}_{i^{\prime}}$ considered as a sequence of smooth sections of $H^{\otimes n} \otimes E$ converges uniformly in the $C^{1}$ topology on any compact subset of $X \backslash \Lambda$.

Proof. Since $A_{i}$ is the Hermitian-Einstein connection of the holomorphic vector bundle $\left(E, \bar{\partial}_{A_{i}}\right)$, and $e_{i}^{\alpha}$ are holomorphic sections of $\left(H^{\otimes n} \otimes E, \bar{\partial}_{A_{i}}\right)$, the Bochner-Weitzenbock formula asserts that for some constant $C$ independent of $i$ and $\alpha$,

$$
\Delta_{\mathbf{g}}\left|e_{i}^{\alpha}\right|^{2} \geq-C\left|e_{i}^{\alpha}\right|^{2}
$$

where $\Delta_{\mathrm{g}}$ is the Laplacian operator. Then, since $\left\|e_{i}^{\alpha}\right\|_{L^{2}}=1$, we have the uniform supreme estimate

$$
\sup _{X}\left|e_{i}^{\alpha}\right| \leq C^{\prime}
$$

For any compact subset $K \subset X \backslash \bigwedge$, let $K_{1}$ be another compact set, $K \subset$ $K_{1}^{\text {int }} \subset K_{1} \subset X \backslash \Lambda$. By Lemma 4.5, $A_{i \mid K_{1}} \stackrel{C^{1}}{\rightarrow} \varphi^{*} A_{0 \mid K_{1}}$. Since $e_{i}^{\alpha}$ are $\bar{\partial}_{A_{i}}$ holomorphic on $K_{1}$ and $\varphi^{*} A_{0 \mid K_{1}}$ is smooth, $\left\{e_{i}^{\alpha}\right\}_{i}$ are uniformly bounded in the $C^{2}$ topology on $K$. Then a subsequence $\left\{e_{i^{\prime}}^{\alpha}\right\}_{i^{\prime}}$ will converge on $K$ in the $C^{1}$ topology. A diagonal tracing technique shows that a subsequence $\left\{i^{\prime}\right\} \subset\{i\}$ can be found so that on any compact subset of $X \backslash \Lambda$ and any $1 \leq \alpha \leq N$,

$$
\lim _{i^{\prime}} e_{i^{\prime}}^{\alpha}=e_{0}^{\alpha}
$$

q.e.d.

By the homomorphism $\varphi:(E, h)_{\mid X \backslash \Lambda} \rightarrow\left(E_{0}, h_{0}\right)_{\mid X \backslash \Lambda}$, the sections $e_{0}^{\alpha}$ can be considered as holomorphic sections of $H^{\otimes n} \otimes E_{0}$ on $X \backslash \Lambda$ under the holomorphic structure $\bar{\partial}_{A_{0}}$. Since $A_{0}$ is regular, by Hartogs lemma, $e_{0}^{\alpha}$ extends to holomorphic sections of $H^{\otimes n} \otimes E_{0}$ over $X$. Thanks to (4.6) 
and Fatou's lemma,

$$
\lim _{i^{\prime}} \int\left\langle e_{i^{\prime}}^{\alpha}, e_{i^{\prime}}^{\beta}\right\rangle_{h} * 1=\int\left\langle e_{0}^{\alpha}, e_{0}^{\beta}\right\rangle * 1
$$

Thus the limit sections $e_{0}^{\alpha}, \alpha=1, \cdots, N$, are linear independent holomorphic sections of $H^{\otimes n} \otimes E_{0}$. We let $e_{0}: \bigoplus^{N} H^{\otimes(-n)} \rightarrow E_{0}$ be the homomorphism induced by sections $\left\{e_{0}^{\alpha}\right\}_{\alpha=1}^{N}$ and let $\bar{E}_{0} \subset E_{0}$ be the sheaf $\bar{E}_{0}=\operatorname{Im}\left\{e_{0}\right\}$. We claim that $\operatorname{det} \bar{E}_{0}=\mathscr{O}$ and $c_{2}\left(\bar{E}_{0}\right) \leq d$. Assume not, then we can find a subsheaf $F \subset E_{0}$ so that $\bar{E}_{0} \subset F$, det $F=\mathscr{O}$, and $c_{2}(F)=d$. Since $E_{0}$ is $\mu$-semistable, $F$ is $\mu$-semistable. By our choice of $n, h^{0}\left(H^{\otimes n} \otimes F\right)=N$ and $H^{0}\left(H^{\otimes n} \otimes F\right)$ generates $H^{\otimes n} \otimes F$. Since $H^{0}\left(H^{\otimes n} \otimes \bar{E}_{0}\right) \subset H^{0}\left(H^{\otimes n} \otimes F\right)$ and $h^{0}\left(H^{\otimes n} \otimes \bar{E}_{0}\right) \geq N, \bar{E}_{0}=F$. So the claim is established. We have the following two propositions.

Proposition 4.7. With the notation as above, if the $\gamma\left(F_{i}\right)$ converges, then $c_{2}\left(\bar{E}_{0}\right)=d$ and $\gamma\left(F_{i}\right) \rightarrow \gamma_{\mathscr{Q}}\left(\bar{E}_{0}\right)$, where $\bar{E}_{0}$ is considered as a $\mu$ semistable quotient sheaf given by $e_{0}: \bigoplus^{N} H^{\otimes(-n)} \rightarrow \bar{E}_{0}$.

Proposition 4.8. With the notation as above, assume that $A_{i} \rightarrow\left[A_{0}, \delta\right]$ in the sense of Uhlenbeck's weak compactness theorem, where $\delta \in S^{d-d_{0}} X$ is an integer valued function on $X$ having total value $d-d_{0}$. Then $\delta(x)=$ length $\left(E_{0} \bar{E}_{0}\right)_{x}$.

Proof of Proposition 4.4 (assuming Propositions 4.7 and 4.8). Let $\left\{F_{i}\right\}$ in $\mathscr{M}_{d}^{\mu}$ be any sequence of $\mu$-stable vector bundles such that $\lim \gamma\left(F_{i}\right)=s_{0}$ in $\gamma\left(\overline{\mathscr{M}}_{d}^{\mu}\right)$ and $\lim \sigma \circ \gamma\left(F_{i}\right)=t_{0}$. Then, by Proposition 4.7, $s_{0}=\gamma_{\mathscr{Q}}\left(\bar{E}_{0}\right)$. Note that under the map $\bar{\sigma}, \bar{\sigma}\left(\gamma_{\mathscr{Q}}\left(\bar{E}_{0}\right)\right)=\left(E_{0}, A_{0}, l_{\bar{E}_{0}}\right) \in \overline{\mathscr{N}}_{d}$. Applying Proposition 4.8, we conclude that $\bar{\sigma}\left(\gamma_{\mathscr{Q}}\left(\bar{E}_{0}\right)\right)=t_{0}$. q.e.d.

We first prove Proposition 4.7. We denote by $e_{i}$ the homomorphism $\bigoplus^{N} H^{\otimes(-n)} \rightarrow E$ induced by sections $\left\{e_{i}^{\alpha}\right\}_{\alpha=1}^{N}$, and by $\varepsilon_{i}$ the surjective homomorphisms $\bigoplus^{N} H^{\otimes(-n)} \rightarrow F_{i}$ such that $\varphi_{i} \circ \varepsilon_{i}=e_{i}$ :

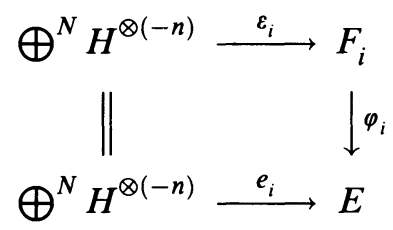

$\varepsilon_{i}$ can be thought of as a sequence of closed points in the Grothendieck's Quot-scheme Quot ${ }_{W}^{\chi}$, where $W=\bigoplus^{N} H^{\otimes(-n)}$ and $\chi=\chi_{E}$ is the Hilbert polynomial of $E$. Since $\operatorname{Quot}_{W}^{\chi}$ is a projective scheme [9, Theorem 3.1], the underlining analytic space $\operatorname{Quot}_{W}^{\chi, \text { red }}$ is compact. Hence, by passing to 
a subsequence, we can assume the $\varepsilon_{i}$ converge to $\varepsilon_{0}: \bigoplus^{N} H^{\otimes(-n)} \rightarrow F_{0}$, where $F_{0}$ is a sheaf with $\operatorname{det} F_{0}=\mathscr{O}$ and $c_{2}\left(F_{0}\right)=d$.

To compare the two limit sheaves $\bar{E}_{0}$ and $F_{0}$, we introduce the induced metrics on quotient sheaves. Let $F$ be any quotient sheaf of $\bigoplus^{N} H^{\otimes(-n)}$ and let $Q$ be the kernel of the homomorphism $\beta: \bigoplus^{N} H^{\otimes(-n)} \rightarrow F$. Then we get an exact sequence

$$
0 \rightarrow Q \rightarrow \bigoplus^{N} H^{-n} \rightarrow F \rightarrow 0
$$

On the open subset $U \subset X$ where $F$ is locally free, (4.9) is an exact sequence of vector bundles. Then, by identifying $F_{\mid U}$ with $Q_{\mid U}^{\perp} \subset$ $\bigoplus^{N} H^{\otimes(-n)}$ and using a fixed Hermitian metric on $\bigoplus^{N} H^{\otimes(-n)}$, we get a Hermitian metric $g$ on $F_{\mid U}$. We call $g$ the quotient metric of $F$. Note that $g$ is only defined on where $F$ is locally free.

Lemma 4.9. There is a constant $C$ so that if we let $g_{i}$ be the quotient metrics of $\varepsilon_{i}: \bigoplus^{N} H^{\otimes(-n)} \rightarrow F_{i}$, then $\left|\varphi_{i}(v)\right|_{h(x)} \leq C|v|_{g_{i}(x)}$ for any $x \in X$ and any $v \in F_{i} \otimes k(x)$.

Proof. Since the supremum norm of $e_{i}^{\alpha} \in H^{0}\left(H^{\otimes n} \otimes E\right)$ is bounded from above by a constant $C$, the supreme norm of the homomorphism $e_{i}: \bigoplus^{N} H^{\otimes(-n)} \rightarrow E$ is bounded from above by $N C$. Therefore, for the corresponding quotient metric $g_{i}^{\prime}$ on $E$ and $u \in E \otimes k(x)$,

$$
|u|_{h(x)} \leq N C|u|_{g_{i}^{\prime}(x)} \text {. }
$$

Since $\varphi_{i} \circ \varepsilon_{i}=e_{i}$ and $g_{i}$ (resp. $g_{i}^{\prime}$ ) are the quotient metrics corresponding to $\varepsilon_{i}$ (resp. $\left.e_{i}\right)$, then $\left|\varphi_{i}(u)\right|_{g_{i}^{\prime}(x)}=|u|_{g_{i}(x)}$. Combined with (4.10), we have proved the desired inequality. q.e.d.

Next we shall show that there exists a homomorphism $\varphi_{0}: F_{0} \rightarrow \bar{E}_{0}$ which is an isomorphism at generic points of $X$. Let $\operatorname{ker}\left(\varepsilon_{0}\right)$ (resp. $\left.\operatorname{ker}\left(e_{0}\right)\right)$ be the kernel of $\varepsilon_{0}$ (resp. $e_{0}$ ). We have the following diagram:

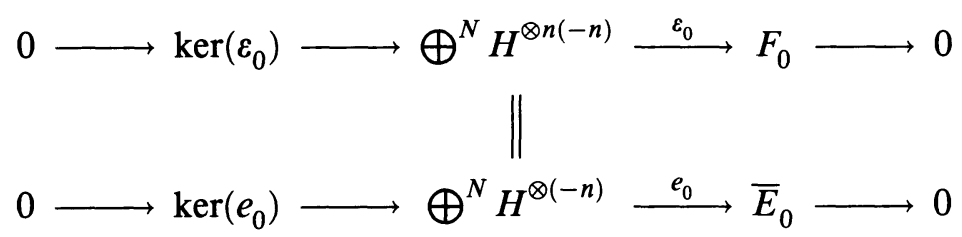

If we can show that in this diagram,

$$
\operatorname{ker}\left(\varepsilon_{0}\right) \subset \operatorname{ker}\left(e_{0}\right)
$$

as subsheaves of $\bigoplus^{N} H^{\otimes(-n)}$, then $\varphi_{0}$ exists and $\varphi_{0} \circ \varepsilon_{0}=e_{0}$. 
Since both $\operatorname{ker}\left(\varepsilon_{0}\right)$ and $\bar{E}_{0}$ are torsion free, (4.11) holds if it holds at generic points of $X$. Let $x \in X$ be any point where $F_{0}$ and $\bar{E}_{0}$ are locally free. For any $v \in \operatorname{ker}\left(\varepsilon_{0}\right) \otimes k(x)$,

$$
\lim \left|\varepsilon_{i}(v)\right|_{g_{i}(x)}=0 \text {, }
$$

which can be seen as follows. Let $U \subset$ Quot $_{W}^{\chi}$ be an analytic neighborhood of the quotient sheaf $\bigoplus^{N} H^{\otimes(-n)} \rightarrow F_{0}$ and let $F$ be the universal quotient sheaf on $X \times U$. Since $F_{0}$ is locally free at $x$, there is an open neighborhood $V \subset X$ of $x$ and an open neighborhood $U^{\prime} \subset U$ of $\left\{\varepsilon_{0}\right\}$ such that $F$ is locally free on $V \times U^{\prime}$. Let $Q$ be the kernel of $\bigoplus^{N} p_{1}^{*} H^{\otimes(-n)} \rightarrow F$ over $V \times U^{\prime}$. Then

$$
0 \rightarrow Q \rightarrow \bigoplus^{N} p_{1}^{*} H^{\otimes(-n)} \rightarrow F \rightarrow 0
$$

is an exact sequence of vector bundles on $V \times U^{\prime}$. Hence, for any $\omega$ in $U^{\prime}$ with the corresponding quotient homomorphism $e_{\omega}: \bigoplus^{N} H^{\otimes(-n)} \rightarrow$ $F \otimes k(\omega)$, and for any $v \in \bigoplus^{N} H^{\otimes(-n)} \otimes k(x)$ with $x \in V$,

$$
\lim _{\omega \rightarrow \omega_{0}} e_{\omega}(v)=e_{\omega_{0}}(v), \omega_{0} \in U^{\prime} .
$$

In particular, since we assume that $\varepsilon_{i}: \bigoplus^{N} H^{\otimes(-n)} \rightarrow F_{i}$ converges to $\varepsilon_{0}: \bigoplus^{N} H^{\otimes(-n)} \rightarrow F_{0}$ in Quot $_{W}^{\chi}$, we have for large $i,\left\{\varepsilon_{i}: \bigoplus^{N} H^{\otimes(-n)} \rightarrow\right.$ $\left.F_{i}\right\} \in U^{\prime}$ and then for any $v \in \operatorname{ker}\left(\varepsilon_{0}\right) \otimes k(x)$,

$$
\lim _{i \rightarrow \infty} \varepsilon_{i}(v)=\varepsilon_{0}(v)=0 .
$$

Then (4.12) follows easily from the fact that $\lim g_{i}=g_{0}$ over $V$.

Now we apply Lemma 4.9 to $v \in \operatorname{ker}\left(\varepsilon_{0}\right) \otimes k(x) \subset \bigoplus^{N} H^{\otimes(-n)} \otimes k(x)$ over a generic point $x \in X$. We obtain $\lim \left|\varphi_{i} \circ \varepsilon_{i}(v)\right|_{h(x)}=0$. Finally,

$$
\lim \varphi_{i} \circ \varepsilon_{i}(v)=\lim e_{i}(v)=e_{0}(v) .
$$

Therefore, $\left|e_{0}(v)\right|_{h(x)}=0$ or $v \in \operatorname{ker}\left(e_{0}\right) \otimes k(x)$. Since $x$ is generic, we have proved $\operatorname{ker}\left(\varepsilon_{0}\right) \subset \operatorname{ker}\left(e_{0}\right)$.

Let $\varphi_{0}: F_{0} \rightarrow \bar{E}_{0}$ be the induced homomorphism.

Lemma 4.10. With the notation as before, let $\varphi_{0}: F_{0} \rightarrow \bar{E}_{0}$ be the induced homomorphism from (4.11). Then $\varphi_{0}$ is an isomorphism.

Proof. Since $\operatorname{rank}\left(\operatorname{ker}\left(\varepsilon_{0}\right)\right)=\operatorname{rank}\left(\operatorname{ker}\left(e_{0}\right)\right)$, it follows that $\operatorname{ker}\left(\varepsilon_{0}\right) \otimes$ $\mathscr{O}_{X, x}=\operatorname{ker}\left(e_{0}\right) \otimes \mathscr{O}_{X, x}$ as subsheaves of $\bigoplus^{N} H^{\otimes(-n)}$ at generic points of $X$. Let $T \subset F_{0}$ be the torsion subsheaf and let $\operatorname{ker}\left(\bar{\varepsilon}_{0}\right)$ be the kernel of the induced homomorphism $\bar{\varepsilon}_{0}: \bigoplus^{N} H^{\otimes(-n)} \rightarrow F_{0} / T$. Since $T$ is supported 
at a subvariety of $X$, we have $\operatorname{ker}\left(\bar{\varepsilon}_{0}\right) \otimes \mathscr{O}_{X, x}=\operatorname{ker}\left(e_{0}\right) \otimes \mathscr{O}_{X, x}$ at the generic points of $X$. Then $\bar{\varepsilon}_{0}\left(\operatorname{ker}\left(e_{0}\right)\right)$ (resp. $\left.e_{0}\left(\operatorname{ker}\left(\bar{\varepsilon}_{0}\right)\right)\right)$ is a torsion subsheaf of $F_{0} / T$ (resp. $\left.\bar{E}_{0}\right)$ and hence $\bar{\varepsilon}_{0}\left(\operatorname{ker}\left(e_{0}\right)\right)\left(\right.$ resp. $\left.e_{0}\left(\operatorname{ker}\left(\bar{\varepsilon}_{0}\right)\right)\right)$ is trivial. So there is a homomorphism $\varphi: F_{0} / T \rightarrow \bar{E}_{0}$ (resp. $\phi: \bar{E}_{0} \rightarrow F_{0} / T$ ) that is an isomorphism on the generic point of $X$. As a consequence, $\chi_{\bar{E}_{0}}=\chi_{F_{0} / T}$. Since $\operatorname{det} \bar{E}_{0}=\operatorname{det} F_{0}, T$ is supported at a discrete point set of $X$. Thus, $c_{2}\left(F_{0} / T\right)=c_{2}\left(F_{0}\right)+$ length $(T)=d+$ length $(T)$. But we know $c_{2}\left(\bar{E}_{0}\right) \leq d$. So length $(T)=0$. The lemma is established.

Proof of Proposition 4.7. By the previous lemmas, for the sequence $\left\{F_{i}\right\} \subset \mathscr{M}_{d}^{\mu}$, there is a subsequence $\left\{i^{\prime}\right\} \subset\{i\}$ with surjections $\varepsilon_{i^{\prime}}$ : $\bigoplus^{N} H^{\otimes(-n)} \rightarrow F_{i^{\prime}}$ such that if we denote by $\left\{F_{i^{\prime}}\right\}$ the corresponding quotient sheaves, then $\lim _{i^{\prime}}\left\{F_{i^{\prime}}\right\}=\left\{F_{0}\right\}$ in Quot ${ }_{W}^{\chi}$ with $F_{0} \cong \bar{E}_{0}$.

Since $F_{0}$ is $\mu$-semistable, $\lim _{i^{\prime}}\left\{F_{i^{\prime}}\right\}=\left\{F_{0}\right\}$ implies $\gamma_{\mathscr{Q}}\left(\bar{E}_{0}\right)=\gamma_{\mathscr{Q}}\left(F_{0}\right)=$ $\lim _{i^{\prime}} \gamma_{\mathscr{Q}}\left(F_{i^{\prime}}\right)=\lim _{i^{\prime}} \gamma\left(F_{i^{\prime}}\right)$. Hence the proposition is proved. q.e.d.

For the proof of Proposition 4.8, we first give a geometric interpretation of the Dirac measure $\delta$ in Uhlenbeck's compactification $\overline{\mathscr{N}}_{d}$. In the following, we denote by $B_{\epsilon}(x)$ the closed $\epsilon$-ball of $X$ centered at $x$ and denote by $\partial B_{\epsilon}(x)$ the boundary of $B_{\epsilon}(x)$.

Lemma 4.11. With the notation as in Lemma 4.5, for any $x$, where $\delta_{x} \neq 0$, let $B_{\epsilon}(x)$ be a ball of sufficiently small radius $\epsilon$. The trivialization of $E_{0 \mid \partial B_{\epsilon}(x)}$ which is induced from a trivialization of $E_{0 \mid B_{\epsilon}(x)}$ induces a trivialization $\beta: \mathbb{C}^{\otimes 2} \times \partial B_{\epsilon}(x) \cong E_{\mid \partial B_{\epsilon}(x)}$ by using the isomorphism $\varphi: E_{\mid X \backslash \Lambda} \rightarrow E_{0 \mid X \backslash \Lambda}$ as in Lemma 4.5. If we denote by $E_{\mid B_{\epsilon}(x)} / E_{\mid \partial B_{\epsilon}(x)}$ the complex vector bundle on $S^{4} \cong B_{\epsilon}(x) / \partial B_{\epsilon}(x)$ derived by identifying $E_{\mid \partial B_{\epsilon}(x)}$ to $\mathbb{C}^{\otimes 2}$ using $\beta$, then

$$
\delta(x)=c_{2}\left(E_{\mid B_{\epsilon}(x)} / E_{\mid \partial B_{\epsilon}(x)}\right) .
$$

Proof. In [7, §3], Friedman and Morgan showed that $\delta(x)$ is equal to the second Chern class of a vector bundle on $S^{4}$. It is easy to see that their construction of the vector bundle is parallel to the procedure described in the lemma. Therefore,

$$
\delta(x)=c_{2}\left(E_{\mid B_{\epsilon}(x)} / E_{\mid \partial B_{\epsilon}(x)}\right) .
$$

q.e.d.

For our application, we need the following lemma.

Lemma 4.12. Let $B^{2} \subset \mathbb{C}^{2}$ (resp. $D \subset \mathbb{C}$ ) be the unit ball and let $\epsilon$ be a sufficiently small positive number. Assume that $F \rightarrow B^{2} \times D$ is a family of torsion free (analytic) sheaves of rank two on $B^{2} \times D$ flat over $D$ and that $F$ is locally free on $B^{2} \times D \backslash(0,0)$. Let $B_{\epsilon, u}^{2}(0) \subset B^{2} \times\{u\}$ be 
the two-dimensional $\epsilon$-ball centered at $(0, u)$ and let $F_{u}$ be the restriction of $F$ to $B_{\epsilon, u}^{2}(0)$. Then the smooth trivialization of $F_{0 \mid \partial B_{\epsilon, 0}^{2}(0)}$ which is induced from a trivialization of $\left(F_{0 \mid B_{\epsilon, 0}^{2}(0)}\right)^{\vee \vee}$ induces a family of trivializations $\beta_{u}: \mathbb{C}^{\otimes 2} \times \partial B_{\epsilon, u}^{2} \cong F_{u \mid \partial B_{\epsilon, u}^{2}(0)}$ which depend smoothly on $u$ with $u \in D$ small. Similar to Lemma 4.11 , we can view $F_{u \mid B_{\epsilon, u}^{2}(0)} / F_{u \mid \partial B_{\epsilon, u}^{2}(0)}$ as a smooth vector bundle over $S^{4} \cong B_{\epsilon, u}^{2}(0) / \partial B_{\epsilon, u}^{2}(0)$ by identifying $F_{u \mid \partial B_{\epsilon, u}^{2}(0)}$ to $\mathbb{C}^{2}$ using the given trivialization. Then

$$
c_{2}\left(F_{u \mid B_{\epsilon, u}^{2}(0)} / F_{u \mid \partial B_{\epsilon, u}^{2}(0)}\right)=\text { length }\left(F_{0}^{\vee \vee} \backslash F_{0}\right)_{0} .
$$

Proof. We prove the lemma by induction on the length of $F_{0}^{\vee \vee} / F_{0}$. First we check the case where length $\left(F_{0}^{\vee \vee} / F_{0}\right)_{0}=1$. Let $(y, z)$ (resp. $u$ ) be the complex coordinates of $B^{2}$ (resp. $D$ ). Then $F_{0}$ is of the form $\mathscr{O} \oplus I_{0}$, where $I_{0}=(y, z) \mathscr{O}_{B^{2}}$ is the ideal sheaf of $0 \in B^{2}$. Since $F$ is flat over $D, F$ fits into the exact sequence

$$
0 \rightarrow \mathscr{O} \stackrel{f}{\longrightarrow} \mathscr{O}^{\oplus 3} \stackrel{g}{\longrightarrow} F \rightarrow 0
$$

with $f=\left(u^{m} f_{1}, z f_{2}, y f_{3}\right)$, where $f_{i}$ are holomorphic functions on $B^{2} \times$ $D$ with $f_{i}(0,0) \neq 0$ for $i=1,2,3$. Without loss of generality, we can assume $f_{i} \equiv 1$ for $i=1,2,3$. We now check for $\epsilon, u$ small,

$$
1=c_{2}\left(F_{u \mid B_{\epsilon, u}^{2}(0)} / F_{u \mid \partial B_{\epsilon, u}^{2}(0)}\right) .
$$

Let $v=(1,0,0) \in \mathscr{O}^{\oplus 3}$ be the section over $B^{2} \times D$. Clearly $g(v)_{\mid B^{2} \times\{0\}}=$ $(1,0) \in \mathscr{O} \oplus \mathscr{J}_{0}$. So under the obvious trivialization $\beta_{0}: \mathbb{C}^{2} \times \partial B_{\epsilon}(0) \rightarrow$ $F_{0 \mid \partial B_{\epsilon}(0)}, g(v)_{\mid \partial B_{\epsilon}(0)}$ is a nonzero constant section on $\partial B_{\epsilon, 0}^{2}(0)$. Now we choose a continuous family of trivializations $\beta_{u}$ so that the section $g(v)_{\mid \partial B_{\epsilon, u}(0)}$ is still constant on $\partial B_{\epsilon, u}^{2}(0)$ under $\beta_{u}$. So $g(v)_{\mid B_{\epsilon, u}}(0)$ can be pushed down to a well-defined section $\tilde{g}(v)_{u} \in F_{u \mid B_{\epsilon, u}^{2}(0)} / F_{u \mid \partial B_{\epsilon, u}^{2}(0)}$. For $|u|$ small, $g(v)_{\mid \partial B_{\epsilon, u}^{2}(0)} \neq 0$. Thanks to the explicit expression that $f=\left(u^{m}, z, y\right), \tilde{g}(v)_{u}$ vanishes at $(0,0)$ only and the contribution of this zero to the $c_{2}\left(F_{u \mid B_{\epsilon, u}^{2}(0)} / F_{u \mid \partial B_{\epsilon, u}^{2}(0)}\right)$ is 1 since $\tilde{g}(v)_{u}$ is transverse to the zero section, since $\tilde{g}(v)_{u}$ is holomorphic and since $\epsilon$ is sufficiently small. So the lemma is established when length $\left(F_{0}^{\vee \vee} / F_{0}\right)_{0}=1$.

Now assume that the lemma holds when length $\left(F_{0}^{\vee \vee} / F_{0}\right)_{0} \leq k$, and that the sheaf $F_{0}$ has length $\left(F_{0}^{\vee \vee} / F_{0}\right)=k+1$. Then we have an exact 
sequence similar to (4.15) on $B^{2} \times D$ :

$$
0 \rightarrow \mathscr{O}^{p} \stackrel{\alpha}{\longrightarrow} \mathscr{O}^{\oplus p+2} \rightarrow F \rightarrow 0 .
$$

By Proposition 6.3 in the Appendix, we can find a deformation $G$ of $F_{0}$ on $B^{2} \times D^{\prime}, D^{\prime} \subset \mathbb{C}$, such that $\left(G^{\vee \vee}\right)_{v} \cong F_{0}^{\vee \vee}$ for all $v \in D^{\prime} \subset \mathbb{C}^{1}$ and length $\left(G_{v}^{\vee \vee} / G_{v}\right)_{x} \leq k$ for any $x \in B_{\epsilon, v}^{2}(0)$ when $v \neq 0$. Since $G$ is a flat family of torsion free sheaves, there is an exact sequence on $B^{2} \times D^{\prime}$,

$$
0 \rightarrow \mathscr{O}^{p} \stackrel{\alpha^{\prime}}{\longrightarrow} \mathscr{O}^{\oplus p+2} \rightarrow G \rightarrow 0,
$$

such that $\alpha^{\prime}(y, z, 0)=\alpha(y, z, 0)$. Now consider a new matrix valued holomorphic function $f$ on $B^{2} \times D \times D^{\prime}$ such that $f(y, z, u, 0)=$ $\alpha(y, z, u)$ and $f(y, z, 0, v)=\alpha^{\prime}(y, z, v)$. Such a holomorphic function does exist. $f$ induces an exact sequence over $B^{2} \times D \times D^{\prime}$,

$$
0 \rightarrow \mathscr{O}^{p} \stackrel{f}{\longrightarrow} \mathscr{\mathscr { O }}^{\oplus p+2} \rightarrow E \rightarrow 0 .
$$

Since $E_{\mid B^{2} \times\{0,0\}}=F_{0}$ is torsion free, $E_{\mid B_{1 / 2}^{2} \times\{u, v\}}$ is torsion free for $u, v$ sufficiently small. Further, $E_{\mid B^{2} \times D \times\{0\}}=F$ and $E_{\mid B^{2} \times\{0\} \times D^{\prime}}=G$. Let $\Sigma \subset B^{2} \times D \times D^{\prime}$ be the set of points where $E$ is not locally free. Since $\Sigma \cap B^{2} \times D \times\{0\}=\{(0,0,0)\}, \Sigma$ is a curve. Hence for any $v \neq 0$, $|v|$ sufficiently small, $E_{\mid B_{1 / 2}^{2} \times\{u\} \times\{v\}}$ is locally free for generic $u \in D$. Further, for any such $u, v$, there is a path in $D \times\{v\}$ connecting $(0, v)$ and $(u, v)$ such that any $\left(u^{\prime}, v\right) \neq(0, v)$ in this path has the property that $E_{\mid B_{1 / 2}^{2} \times\left\{u^{\prime}\right\} \times\{v\}}$ is locally free. Finally, let $(s, t) \in[0,1] \times[0,1]$ be real parameters and let $\rho:[0,1] \times[0,1] \rightarrow D \times D^{\prime}$ be a family of paths having the properties: $\rho(0,0)=(0,0), \rho((0,1] \times\{0\}) \subset(D \backslash 0) \times\{0\}$, $\rho(\{0\} \times(0,1]) \subset\{0\} \times\left(D^{\prime} \backslash 0\right), \rho((0,1] \times(0,1]) \subset(D \backslash 0) \times\left(D^{\prime} \backslash 0\right)$, and $\Sigma \cap B_{1 / 2}^{2} \times\{\rho(s, t)\}=\varnothing$ for any $(s, t) \in(0,1] \times[0,1]$. Thus, $E_{\mid B_{1 / 2}^{2} \times\{\rho(s, t)\}}$ is locally free whenever $s \neq 0$. In the following, we will only consider those $(u, v) \in D \times D^{\prime}$ that are contained in the image set $\rho([0,1] \times[0,1])$.

Let $B_{\epsilon, u, v}^{2}(0) \subset B^{2} \times\{u\} \times\{v\}$ be the $\epsilon$-ball. Since $\left(E_{\mid B_{1 / 2}^{2} \times\{0\} \times D^{\prime}}\right)^{\vee \vee}$ is locally free, we can assume the trivialization $\beta_{0, v}: \mathbb{C}^{\oplus 2} \times \partial B_{\epsilon, 0, v}^{2}(0) \rightarrow$ $E_{\mid \partial B_{\epsilon, 0, v}^{2}(0)}$, which is induced from the trivialization $\left(E_{\mid B_{\epsilon, 0, v}^{2}(0)}\right)^{\vee V}$, is smooth in variable $v$ when $|v|$ is sufficiently small. Thus for $u, v$ small enough (in $\rho([0,1] \times[0,1]))$, the induced trivializations $\beta_{u, v}: \mathbb{C}^{\oplus 2} \times$ $\partial B_{\epsilon, u, v}^{2}(0) \rightarrow E_{\mid \partial B_{\epsilon, u, v}^{2}(0)}$ can be smooth in $(u, v)$ also. So 


$$
c_{2}\left(E_{\mid B_{\epsilon, u, 0}^{2}(0)} / E_{\mid \partial B_{\epsilon, u, 0}^{2}(0)}\right)=c_{2}\left(E_{\mid \backslash E_{\mid \partial B_{\varepsilon, u, v}^{2}}^{2}(0) B_{\epsilon, u, v}^{2}(0)}\right) .
$$

By assumption, for $(0, v)$ small, there are points $p_{1}, p_{2}, \cdots, p_{l} \in$ $\operatorname{Int}\left(B_{\epsilon, 0, v}^{2}(0)\right)$ (depending on $v$ ) such that $p_{1}, p_{2}, \cdots, p_{l}$ are the only nonlocally free points of $E_{\mid B_{\epsilon, 0, v}^{2}(0)}$. Further, at each of the points $p_{1}, p_{2}$, $\cdots, p_{l}$, length $\left(\left(E_{\mid B_{\epsilon, 0, v}^{2}(0)}\right)^{\vee \vee} / E_{\mid B_{\epsilon, 0, v}^{2}(0)}\right)_{p j} \leq k$. By the induction hypothesis, we can use $\delta$-balls $(\delta \ll \epsilon)$ centered at $p_{1}, p_{2}, \cdots, p_{l}$ and the trivialization $\mathbb{C}^{\oplus 2} \times \partial B_{\delta, u, v}^{2}\left(p_{j}\right) \cong E_{\mid \partial B_{\delta, u, v}^{2}\left(p_{j}\right)}$ induced from $\mathbb{C}^{\oplus 2} \times B_{\delta, 0, v}^{2}(0) \cong$ $\left(E_{\mid B_{\epsilon, 0, v}^{2}(0)}\right)^{\vee \vee}$ to conclude

$$
\begin{aligned}
& \sum_{j=1}^{l} c_{2}\left(E_{\mid B_{\delta, u, v}^{2}\left(p_{j}\right)} / E_{\mid \partial B_{\delta, u, v}^{2}\left(p_{j}\right)}\right) \\
& =\sum_{j=1}^{l} \operatorname{length}\left(\left(E_{\mid B_{\delta, 0, v}^{2}\left(p_{j}\right)}\right)^{\vee \vee} / E_{\mid B_{\delta, 0, v}^{2}\left(p_{j}\right)}\right)_{p_{j}} .
\end{aligned}
$$

Since $E_{\mid B_{\epsilon, u, v}^{2}(0) \backslash \cup B_{\delta, u, v}^{2}\left(p_{j}\right)}$ is a smooth family of vector bundles (in $u$ ), the contribution of its second Chern class with respect to the trivializations on $\partial B_{\epsilon, u, v}^{2}(0)$ and $\partial B_{\delta, u, v}^{2}\left(p_{j}\right)$ for $j=1,2, \cdots, l$ is constant and consequently trivial because $E_{\left|B_{\epsilon, 0, v}^{2}\right| \cup B_{\delta, u, v}^{2}\left(p_{j}\right)}$ is flat. So

$$
\begin{aligned}
c_{2}\left(E_{\mid B_{\epsilon, u, v}^{2}(0)} / E_{\mid \partial B_{\epsilon, u, v}^{2}(0)}\right) & =\sum_{j=1}^{l} c_{2}\left(E_{\mid B_{\delta, u, v}^{2}\left(p_{j}\right)} / E_{\mid \partial B_{\delta, u, v}^{2}\left(p_{j}\right)}\right) \\
& =\sum_{j=1}^{l} \operatorname{length}\left(\left(E_{\mid B_{\delta, 0, v}^{2}\left(p_{j}\right.}\right)^{\vee \vee} / E_{\mid B_{\delta, 0, v}^{2}\left(p_{j}\right)}\right)_{p_{j}} \\
& =\operatorname{length}\left(\left(E_{\mid B_{\epsilon, 0,0}^{2}(0)}\right)^{\vee \vee} / E_{\mid B_{\epsilon, 0,0}^{2}(0)}\right)_{0} .
\end{aligned}
$$

The last equality holds since $E_{\mid B_{\epsilon, 0, v}^{2}}$ is a flat family of torsion free sheaves over $D^{\prime}$ with $E_{\mid B_{\epsilon, 0, v}^{2}}^{\vee \vee} \cong E_{\mid B_{\epsilon, 0,0}^{2}}^{\vee \vee}$. Combined with (4.19), the lemma is established.

Proof of Proposition 4.8. By the proof of Proposition 4.7, we know that the limit $A_{i} \rightarrow\left[A_{0}, \delta\right]$ is compatible with the limit $F_{i} \rightarrow F_{0}$, where $F_{i}, F_{0}$ are considered as quotient sheaves of $\bigoplus^{N} H^{\otimes(-n)}$. Hence from Lemma 4.11 the singular measure

$$
\delta(x)=c_{2}\left(E_{\mid B_{\epsilon}^{2}(x)} / E_{\mid \partial B_{\epsilon}^{2}(x)}\right)
$$

is identical to 


$$
c_{2}\left(F_{i \mid B_{\epsilon}^{2}(x)} / F_{i \mid \partial B_{\epsilon}^{2}(x)}\right)
$$

for $i \gg 0$ when the trivializations are induced from the limit $F_{i} \rightarrow F_{0}$. Then Proposition 4.8 follows from applying Lemma 4.12. q.e.d.

In the sequel, we will view $\overline{\mathscr{N}}_{d}$ as a projective scheme that is isomorphic to $\gamma\left(\overline{\mathscr{M}}_{d}^{\mu}\right)$.

\section{Donaldson's polynomial invariants of algebraic surfaces}

The goal of this section is to redefine Donaldson's polynomial invariants of the underlining smooth four-manifold of an algebraic surface in algebraic geometric context. Loosely speaking, we will show that part of Donaldson's polynomial invariants can be calculated by evaluating the intersection numbers of certain Cartier divisors on the space $\mathscr{M}_{d}$, the coarse moduli scheme of rank-two semistable sheaves on $X$.

For any simply connected oriented smooth four-manifold $X$ with $b_{2}^{+}(X)$ odd and strictly greater than 1 , Donaldson defined a series of integer valued polynomials $\left\{q_{d}\right\}_{k_{0}}^{\infty}$,

$$
q_{d}: H_{2}(X) \times \cdots \times H_{2}(X) \rightarrow \mathbb{Z},
$$

with $\operatorname{deg} q_{d}=4 d-\frac{3}{2} \cdot\left(1+b_{2}^{+}\right) . q_{d}$ is defined as the intersection number of some properly defined subsets in $\overline{\mathscr{N}}_{d}(\mathbf{g})$, where $\mathbf{g}$ is a generic Riemannian metric. For a detailed discussion of Donaldson's polynomial invariants, we refer the reader to Donaldson's original paper [3] or Friedman and Morgan's recent book [7].

Let $H$ be any ample divisor. For any $d>0$, we put

$$
\Omega(H, d)=\{D \in \mathrm{NS}(X) \mid D \cdot H=0, D \cdot D \geq-d\}
$$

and put $\Omega(H, d)^{\perp}=\left\{D \in \mathrm{NS}(X) \mid D \cdot D^{\prime}=0\right.$ for any $\left.D^{\prime} \in \Omega(H, d)\right\}$. We have the following observation.

Lemma 5.1. Let $F \in \mathscr{M}_{d}(H)$. Assume $L \subset F$ with $\operatorname{deg}_{H}(L)=0$. Then $c_{1}(L) \cdot D=0$ for any $D \in \Omega(H, d)^{\perp}$.

Proof. Assume $F \in \mathscr{M}_{d}(H)$ is not $\mu$-stable, and $L \subset F$ is a subsheaf such that $\operatorname{deg}_{H}(L)=0$ and $F / L$ is torsion free. Since $\operatorname{det}(F)=\mathscr{O}$, we have $c_{2}(F) \geq-c_{1}(L)^{2}$. Thus, $c_{1}(L) \in \Omega(H, d)$. So the lemma is established. q.e.d.

By applying Proposition 1.7 to the moduli scheme $\mathscr{M}_{d}(H)$, we construct a homomorphism

$$
\rho_{H}: \Omega(H, d)^{\perp} \rightarrow \mathrm{NS}\left(\mathscr{M}_{d}(H)\right)
$$


In the case $\operatorname{dim} \mathscr{M}_{d}(H)=c(d)$, where $c(d)=4 d-3 \chi(\mathscr{O})$ is the virtual dimension of $\mathscr{M}_{d}(H)$, we define a polynomial

$$
\delta_{d}(H): \operatorname{Symm}^{k}\left(\Omega(H, d)^{\perp}\right) \rightarrow \mathbb{Z},
$$

$k=c(d)$, as follows. For $\alpha_{1}, \cdots, \alpha_{k} \in \Omega(H, d)^{\perp}$,

$$
\delta_{d}(H)\left(\alpha_{1}, \cdots, \alpha_{k}\right)=\left[\rho_{H}\left(\alpha_{1}\right) \cdots \rho_{H}\left(\alpha_{k}\right)\right]\left(\mathscr{M}_{d}(H)\right) .
$$

Theorem 6. Let $X$ be any smooth, simply connected algebraic surface with $p_{g}>0$ and let $H$ be any ample divisor. Then there is a constant $A(H)$ such that for any $d \geq A(H), \delta_{d}(H)$ coincides with the restriction of Donaldson's polynomial invariant $q_{d}$ to $\operatorname{Symm}^{k}\left(\Omega(H, d)^{\perp}\right) \subset$ $\operatorname{Symm}^{k} H^{2}(X, \mathbb{Z})$, where $k=c(d)$.

Proof. We choose $A(H) \geq 2 p_{g}+1$ to be large enough so that whenever $d \geq A(H)$, then $\mathscr{M}_{d}^{\mu}(H)$ is dense in $\mathscr{M}_{d}(H)$ and $\mathscr{M}_{d}(H)$ is smooth at generic points of $\mathscr{M}_{d}(H)$. Thus $\overline{\mathscr{N}}_{d}\left(=\gamma\left(\overline{\mathscr{M}}_{d}^{\mu}(H)\right)\right)$ is isomorphic to the reduction of $\gamma\left(\mathscr{M}_{d}(H)\right)$. We first state a general result concerning Donaldson's polynomial invariants. The proof of it can be found in [3, $\S 5.3]$ or $[7, \S 3.9 .21]$. Let $d \geq A(H)$, let $C_{1}, \cdots, C_{k} \in \Omega(H, d)^{\perp}$ be any smooth divisors, and let $\mathscr{L}_{\mathscr{M}}\left(C_{i}, \theta_{i}\right) \in \rho_{H}\left(C_{i}\right)$ be the associated determinant line bundles (see Proposition 1.7). Assume that there are line bundles $\mathscr{L}_{\mathscr{N}}\left(C_{i}, \theta_{i}\right)$ on $\overline{\mathscr{N}}_{d}(H)$ such that

$$
\gamma^{*} \mathscr{L}_{\mathscr{N}}\left(C_{i}, \theta_{i}\right)=\mathscr{L}_{\mathscr{M}}\left(C_{i}, \theta_{i}\right) \overline{\mathscr{M}}_{d}^{\mu}(H)
$$

via $\gamma: \overline{\mathscr{M}}_{d}^{\mu}(H) \rightarrow \overline{\mathscr{N}}_{d}(H)$. Then

$$
\left[\mathscr{L}_{\mathscr{M}}\left(C_{1}, \theta_{1}\right)\right] \cdots\left[\mathscr{L}_{\mathscr{M}}\left(C_{k}, \theta_{k}\right)\right]=q_{d}\left(\left[C_{1}\right], \cdots,\left[C_{k}\right]\right)
$$

Here $[\cdot] \cdots[\cdot]$ is the intersection of the Cartier divisors in $\overline{\mathscr{M}}_{d}^{\mu}(H)$. Since $\mathscr{L}_{\mathscr{M}}^{\otimes m}\left(D, \theta_{D}\right)$ always descends to a very ample line bundle on $\overline{\mathscr{N}}_{d}(H)$ for $D \in|n H|$, the theorem will be established if we can show that for any $C_{0} \in \Omega(H, d)^{\perp}, \rho_{H}\left(C_{0}+l H\right)$ descends for $l \gg 0$.

Let $\varphi: \mathscr{M}_{d}^{\text {nor }} \rightarrow \mathscr{M}_{d}(H)$ be the normalization of the reduction of $\mathscr{M}_{d}(H)$ and let $\tilde{\varphi}: \overline{\mathscr{N}}_{d}^{\text {nor }} \rightarrow \overline{\mathscr{N}}_{d}(H)$ be the normalization of $\overline{\mathscr{N}}_{d}(H)$. Since $\gamma: \overline{\mathscr{M}}_{d}^{\mu} \rightarrow \overline{\mathscr{N}}_{d}(H)$ is a birational morphism, there is a unique $\tilde{\gamma}: \mathscr{M}_{d}^{\text {nor }} \rightarrow$ $\overline{\mathscr{N}}_{d}^{\text {nor }}$ such that

$$
\tilde{\varphi} \circ \tilde{\gamma}=\gamma \circ \varphi .
$$

Since $\tilde{\varphi}$ and $\varphi$ are birational finite morphisms, the proof of (5.6) [7] shows that the theorem will be proved if the line bundle $\varphi^{*} \mathscr{L}_{\mathscr{M}}\left(C, \theta_{C}\right)$ 
descends to a line bundle on $\overline{\mathscr{N}}_{d}^{\text {nor }}$ via $\tilde{\gamma}: \mathscr{M}_{d}^{\text {nor }} \rightarrow \overline{\mathscr{N}}_{d}^{\text {nor }}$, where $C \in$ $\left|C_{0}+l H\right|$.

Recall the following diagram:

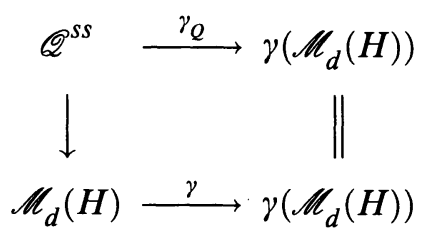

If we let $\varphi_{Q}: \mathscr{Q}_{\text {nor }}^{s s} \rightarrow \mathscr{Q}^{s s}$ be the normalization of the reduction of $\mathscr{Q}^{s s}$, then there is a new diagram compatible to (5.7):

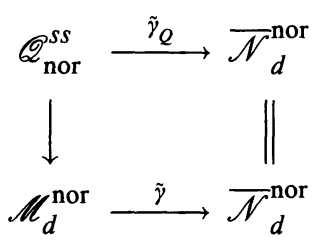

Let $s \in \bar{N}_{d}^{\text {nor }}$ be any closed point. Then there is a $C^{\prime} \in|C|$ and an open neighborhood $V \subset \overline{\mathscr{N}}_{d}^{\text {nor }}$ of $s$ such that whenever $F$ is a quotient sheaf in $\tilde{\gamma}_{Q}^{-1}(V)$, then $F$ is locally free at $C^{\prime}$. Since $l \gg 0, F_{\mid C^{\prime}}$ is semistable. We claim that there is a morphism $p: V \rightarrow \mathscr{M}\left(C^{\prime}\right)$ making the following diagram commutative:

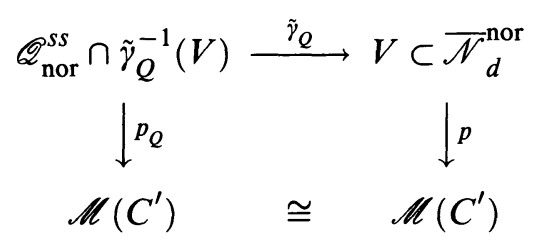

Here $p_{Q}$ is the morphism induced by restricting the universal quotient sheaf to $C^{\prime}$. Indeed, since all schemes involved in (5.9) are normal and $\tilde{\gamma}_{Q}$ is surjective, all we need to show is that $p$ as a map is well defined, making the diagram (5.9) commutative. This is apparent from Theorem 4. Note that

$$
\tilde{\gamma}_{Q}^{*}\left(p^{*} \mathscr{L}_{C^{\prime}}\right)_{\hat{\gamma}_{Q}^{-1}(V)}=p_{Q^{*}}^{*} \mathscr{L}_{C_{\mid \dot{\gamma}_{Q}^{-1}(V)}^{\prime}}=\varphi_{Q}^{*} \mathscr{L}_{\mathscr{Q}}\left(C^{\prime}, \theta_{C^{\prime}}\right)_{\tilde{\gamma}_{Q}^{-1}(V)},
$$

and that $(5.10)$ is $\operatorname{PGL}(N, \mathbb{C})$ equivariant, with $\operatorname{PGL}(N, \mathbb{C})$ acting trivially on $\overline{\mathcal{N}}_{d}^{\text {nor }}$. Thus $(5.10)$ descends to

$$
\varphi^{*} \mathscr{L}_{\mathscr{M}}\left(C^{\prime}, \theta_{C^{\prime}}\right)_{\mid \tilde{\gamma}^{-1}(V)}=\tilde{\gamma}^{*}\left(p^{*} \mathscr{L}_{C^{\prime}}\right)_{\mid \tilde{\gamma}^{-1}(V)}
$$


on $\tilde{\gamma}^{-1}(V)$. Now we cover $\overline{\mathscr{N}}_{d}^{\text {nor }}$ by open sets $V_{1}, \cdots, V_{L}$ so that on each $V_{j}$, there is a line bundle $\mathscr{L}_{j}$ having the property that

$$
\tilde{\gamma}^{*} \mathscr{L}_{j}=\varphi^{*} \mathscr{L}_{\mathscr{M}}\left(C, \theta_{C}\right)_{\mid \tilde{\gamma}^{-1}\left(V_{j}\right)} .
$$

Since $\gamma: \mathscr{M}_{d}^{\mu}(H) \rightarrow \mathscr{N}_{d}(H)$ is an isomorphism (Proposition 3.7), the restriction of the identity homomorphisms

$$
f_{i j}: \varphi^{*} \mathscr{L}_{\mathscr{M}}\left(C, \theta_{C}\right)_{\mid \tilde{\gamma}^{-1}\left(V_{j}\right)} \cong \varphi^{*} \mathscr{L}_{\mathscr{M}}\left(C, \theta_{C}\right)_{\mid \tilde{\gamma}^{-1}\left(V_{i}\right)}
$$

to $\tilde{\gamma}^{-1}\left(V_{j} \cap V_{i}\right) \cap \varphi^{-1}\left(\mathscr{M}_{d}^{\mu}(H)\right) \subset \mathscr{M}_{d}^{\text {nor }}$ induce isomorphisms

$$
\tilde{f}_{i j}: \mathscr{L}_{j_{\mid V_{j} \cap V_{i} \cap N_{d}^{\text {nor }}}} \cong \mathscr{L}_{i_{\mid V_{j} \cap V_{i} \cap N_{d}^{\text {nor }}}}
$$

Since $\operatorname{codim}\left(\overline{\mathscr{N}}_{d}^{\text {nor }} \backslash \mathscr{N}_{d}^{\text {nor }}\right) \geq 2$, the $\tilde{f}_{i j}$ extend to isomorphisms

$$
g_{i j}: \mathscr{L}_{j_{\mid V_{j} \cap V_{i}}} \cong \mathscr{L}_{i_{\mid V_{j} \cap V_{i}}} .
$$

Clearly, $\tilde{\gamma}^{*}\left(g_{i j}\right)=f_{i j}$. So the line bundle $\varphi^{*}\left(\mathscr{L}_{\mathscr{M}}\left(C, \theta_{C}\right)\right)$ descends to the line bundle on $\overline{\mathscr{N}}_{d}^{\text {nor }}$. Thus the theorem is established. q.e.d.

In general, $\Omega(H, d)^{\perp}$ is much smaller than $H^{1,1}(X, \mathbb{Z})$. Indeed, for any fixed $H, \Omega(H, d)^{\perp}=\mathbb{Z}\{H\}$ for $d$ sufficiently large. One solution of this is to consider a more general class of polarizations. Let $\mathrm{NS}(X)_{\mathbb{Q}}$ (resp. $\left.\mathrm{NS}(X)_{\mathbb{R}}\right)$ be the tensor product $\mathrm{NS}(X) \otimes_{\mathbb{Z}} \mathbb{Q}\left(\right.$ resp. $\left.\mathrm{NS}(X) \otimes_{\mathbb{Z}} \mathbb{R}\right)$ and let $\mathrm{NS}(X)_{\mathbb{Q}}^{+}$(resp. $\mathrm{NS}(X)_{\mathbb{R}}^{+}$) be the cone spanned by $\mathrm{NS}(X)^{+}$. For any $\omega_{r} \in \mathrm{NS}(X)_{\mathbb{R}}^{+}$, we define the Hilbert polynomial of a rank-two sheaf $F$ by

$$
\mathscr{X}_{F}(n)=n^{2}\left(\omega_{r} \cdot \omega_{r}\right)+n \omega_{r} \cdot\left(c_{1}(F)-K_{X}\right)+\chi(F) .
$$

The concept of $\omega_{r}$-stability can be defined analogously to the definition of $H$-stability.

Analogously to $\S 1$, we let $\mathscr{E}^{d}\left(\omega_{r}\right)$ be the set of all rank-two sheaves $F$ with $\operatorname{det} F=\mathscr{O}$ and $c_{2}(F)=d$ which are semistable with respect to $\omega_{r}$. We define $\mathscr{F}^{d}\left(\omega_{r}\right)$ to be the functor sending a scheme $S$ to the set of all families of sheaves $F$ in $\mathscr{E}^{d}\left(\omega_{r}\right)$ on $X \times S$ flat over $S$, where two families $F_{1}$ and $F_{2}$ are equivalent if $F_{1} \cong F_{2} \otimes p_{2}^{*} L$ with $L$ a line bundle on $S$.

Corollary 5.2. For any $\omega \in \mathrm{NS}(X)_{\mathbb{Q}}^{+}, \mathscr{F}^{d}(\omega)$ is coarsely represented by a projective scheme $\mathscr{M}_{d}(\omega)$. In particular, if $H$ is an ample divisor representing an integer multiple of $\omega$, then $\mathscr{M}_{d}(\omega) \cong \mathscr{M}_{d}(H)$.

Proof. We simply let $\mathscr{M}_{d}(\omega) \cong \mathscr{M}_{d}(H)$ with $H$ an ample divisor representing an integer multiple of $\omega$. Lemma 5.1 states that $\mathscr{M}_{d}(\omega)$ is 
independent of the choice of $H$. Hence $\mathscr{M}_{d}(\omega)$ is well defined and has the desired properties.

Theorem $6^{\prime}$. Let $X$ be as in Theorem 6. For any compact subcone $\mathscr{C} \subset \mathrm{NS}(X)_{\mathbb{Q}}^{+}$, there is a constant $A(\mathscr{C})$ such that for any $H \in \mathscr{C}$ and any $d \geq A(\mathscr{C})$, the polynomial $\delta_{d}(H)$ is identical to the restriction of Donaldson's polynomial invariant $q_{d}$ to $\operatorname{Symm}^{k}\left(\Omega(H, d)^{\perp}\right) \subset \operatorname{Symm}^{k} H^{2}(X, \mathbb{Z})$, where $k=c(d)$.

Proof. Recall that for any compact cone $\mathscr{C} \subset \mathrm{NS}(X)^{+}$, there is a constant $A(\mathscr{C})$ such that for any $H \in \mathscr{C}$ and any $d \geq(\mathscr{C})$, the moduli scheme $\mathscr{M}_{d}(H)$ satisfies the condition in Theorem 6 [6]. Thus, Theorem $6^{\prime}$ follows from Theorem 6. q.e.d.

In the remainder of this section, we will use a single polarization $\omega_{r} \in$ $\mathrm{NS}(X)_{\mathbb{R}}^{+}$to recover Donaldson's polynomial invariants. We need the following result.

Proposition 5.3. For any $\omega_{r} \in \mathrm{NS}(X)_{\mathbb{R}}^{+}, \mathscr{F}^{d}\left(\omega_{r}\right)$ is coarsely represented by a unique projective scheme $\mathscr{M}_{d}\left(\omega_{r}\right)$.

Proof. The intersection matrix $\langle\cdot, \cdot \cdot\rangle: \mathrm{NS}(X)_{\mathbb{Q}} \times \mathrm{NS}(X)_{\mathbb{Q}} \rightarrow \mathbb{Q}$ is a nondegenerate symmetric matrix with rational entries. For $\omega_{r} \in \mathrm{NS}(X)_{\mathbb{R}}^{+}$, let

$$
\left\langle\omega_{r}, \cdot\right\rangle: \mathrm{NS}(X)_{\mathbb{Q}} \rightarrow \mathbb{R}
$$

be the linear functional, let $\Omega\left(\omega_{r}\right)_{\mathbb{Q}}$ be the kernel of $\left\langle\omega_{r}, \cdot\right\rangle$ in $\mathrm{NS}(X)_{\mathbb{Q}}$, and let $\Omega\left(\omega_{r}\right)_{\mathbb{R}}^{\perp}$ be the orthogonal complement of $\Omega\left(\omega_{r}\right)_{\mathbb{R}}=\Omega\left(\omega_{r}\right)_{\mathbb{Q}} \otimes_{\mathbb{Q}} \mathbb{R}$ in $\left(\operatorname{NS}(X)_{\mathbb{R}},\langle\cdot, \cdot\rangle\right)$. Fix a Euclidean norm $\|\cdot\|$ on $\mathrm{NS}(X)_{\mathbb{R}}$. Define

$$
C_{\epsilon}(\omega)=\left\{\tilde{\omega}=\omega+\alpha \mid \alpha \in \Omega\left(\omega_{r}\right)_{\mathbb{R}}^{\perp},\|\alpha\|<\epsilon\right\} .
$$

One notes that whenever $D \in \operatorname{NS}(X)$ with $\omega \cdot D=0$, then $\omega^{\prime} \cdot D=0$ for any $\omega^{\prime} \in C_{\epsilon}(\omega)$. We claim that for any $\epsilon>0$,

$$
C_{\epsilon}\left(\omega_{r}\right) \cap \mathrm{NS}(X)_{\mathbb{Q}} \neq \varnothing .
$$

We prove the claim by induction on the dimension of $\Omega\left(\omega_{r}\right)_{\mathbb{R}}^{\perp}$. Assume $\Omega\left(\omega_{r}\right)_{\mathbb{R}}^{\perp}=\mathbb{R}\left\{\omega_{r}\right\}$. Then by the nondegeneracy of the intersection matrix, a multiple of $\omega_{r}$ is in $\mathrm{NS}(X)_{\mathbb{Q}}^{+}$. Then the claim holds. Assume $\operatorname{dim} \Omega\left(\omega_{r}\right)_{\mathbb{R}}^{\perp}=k>1$. Then we can find a $\lambda_{1} \in \Omega\left(\omega_{r}\right)_{\mathbb{R}}^{\perp}$, $\left\|\lambda_{1}\right\|<\epsilon / 2$, and an $\alpha \in \mathrm{NS}(X)_{\mathbb{Q}} \mid \Omega\left(\omega_{r}\right)_{\mathbb{Q}}$ so that $\left\langle\alpha, \lambda_{1}+\omega_{r}\right\rangle=0$. Clearly $\mathbb{Q}\{\alpha\} \oplus \Omega\left(\omega_{r}\right)_{\mathbb{Q}} \subseteq \Omega\left(\omega_{r}+\lambda_{1}\right)_{\mathbb{Q}}$. So $\Omega\left(\omega_{r}+\lambda_{1}\right)_{\mathbb{R}}^{\perp} \nsubseteq \Omega\left(\omega_{r}\right)_{\mathbb{R}}^{\perp}$. By the induction hypothesis, we can find $\lambda_{2} \in \Omega\left(\omega_{r}+\lambda_{1}\right)_{\mathbb{R}}^{\perp} \subset \Omega\left(\omega_{r}\right)_{\mathbb{R}}^{\perp}$, $\left\|\lambda_{2}\right\|<\epsilon / 2$, so that $\Omega\left(\tilde{\omega}_{r}\right)_{\mathbb{R}}^{\perp}=\mathbb{R}\left\{\tilde{\omega}_{r}\right\}$, where $\tilde{\omega}_{r}=\omega_{r}+\lambda_{1}+\lambda_{2}$. Thus the claim is proved. 
For any $d>0$, two $\omega_{1}, \omega_{2} \in \mathrm{NS}(X)_{\mathbb{R}}$ are said to be $d$-equivalent if $\Omega\left(\omega_{1}, d\right)=\Omega\left(\omega_{2}, d\right)$, where $\Omega(\omega, d)$ is defined by (5.2). It is obvious that for any $\omega \in \mathrm{NS}(X)_{\mathbb{R}}^{+}$, there is an $\epsilon>0$ depending on $d$ having the property that any two classes in $C_{\epsilon}(\omega)$ are mutually $d$-equivalent. One checks directly that the corresponding moduli functors $\mathscr{F}^{d}(\tilde{\omega})$ with $\tilde{\omega} \in C_{\epsilon}(\omega)$ are mutually equivalent. Therefore, for $\omega \in \mathrm{NS}(X)_{\mathbb{R}}^{+}$, we define $\mathscr{M}_{d}(\omega)$ to be one of $\mathscr{M}_{d}\left(\omega_{1}\right)$, where $\omega_{1} \in C_{\epsilon}(\omega) \cap \mathrm{NS}(X)_{\mathbb{Q}}^{+}$with $\epsilon$ sufficiently small. $\mathscr{M}_{d}\left(\omega_{1}\right)$ is a projective scheme coarsely representing the functor $\mathscr{F}^{d}\left(\omega_{1}\right)=\mathscr{F}^{d}(\omega)$. Hence, $\mathscr{M}_{d}(\omega)$ is well defined. q.e.d.

We call an element in $\mathrm{NS}(X)_{\mathbb{R}}^{+}$a Kähler class. A Kähler class $\omega \in$ $\mathrm{NS}(X)_{\mathbb{R}}^{+}$is said to be generic (resp. $d$-generic) if $\Omega(\omega)=\{0\}$ (resp. $\Omega(\omega, d)=\{0\})$. Note that then $\Omega(\omega)^{\perp}=H^{1,1}(X, \mathbb{Z})$. Let $\omega$ be a generic Kähler class and let $\mathscr{M}_{d}(\omega)$ be the moduli scheme of $\omega$-semistable sheaves on $X$. By Proposition 1.7, there is a homomorphism

$$
\rho_{\omega}: H^{1,1}(X, \mathbb{Z}) \rightarrow \mathrm{NS}\left(\mathscr{M}_{d}(\omega)\right) .
$$

When $\operatorname{dim} \mathscr{M}_{d}(\omega)=c(d)$, we define a polynomial

$$
\delta_{d}(\omega): \operatorname{Symm}^{k}\left(H^{1,1}(X, \mathbb{Z})\right) \rightarrow \mathbb{Z}
$$

similar to $\delta_{d}(H)$ in $(5.5)$.

Theorem 6" . Let $X$ be as in Theorem 6. For any generic Kähler class $\omega \in \mathrm{NS}(X)_{\mathbb{R}}^{+}$, there is a constant $A(\omega)$ such that for any $d \geq A(\omega)$, $\delta_{d}(\omega)$ coincides with the restriction of Donaldson's polynomial invariant $q_{d}$ to $\operatorname{Symm}^{k}\left(H^{1,1}(Z, \mathbb{Z})\right) \subset \operatorname{Symm}^{k} H^{2}(X, \mathbb{Z})$, where $k=c(d)$.

We remark that in defining Donaldson's polynomial invariants of fourmanifolds $X$, one has to use the generic Riemannian metric $\mathbf{g}$ to avoid the occurrence of reducible ASD connections in the closure $\bar{N}_{d}(\mathbf{g})$. In this paper, we showed that to the generic Kähler class $\omega \in \mathrm{NS}(X)_{\mathbb{R}}^{+}$and the corresponding moduli scheme $\mathscr{M}_{d}(\omega)$, no reducible ASD connections occur in $\overline{\mathscr{N}}_{d}(\omega)$ except the flat one. Based on these spaces, we have reproduced part of Donaldson's polynomial invariants that are defined on the subspace

$$
\operatorname{Symm}^{k} H^{1,1}(X, \mathbb{Z}) \subset \operatorname{Symm}^{k} H^{2}(X, \mathbb{Z}) .
$$

The advantage of this approach is apparent. When $X$ is an algebraic surface, the space $\mathscr{M}_{d}(\omega)$ is better understood than the space $\overline{\mathscr{N}}_{d}(\mathbf{g})$ when $\mathbf{g}$ is a generic Riemannian metric. Thus one has a better chance to calculate Donaldson's polynomial invariants by working on $\mathscr{M}_{d}(\omega)$ instead of on $\bar{N}_{d}(\mathbf{g})$ as demonstrated by [7]. We further remark that when $\omega \in \operatorname{NS}(X)_{\mathbb{R}}^{+}$ 
is a generic Kähler class, then any $(E, A, \delta) \in \overline{\mathscr{N}}_{d}(\omega)$ with $(E, A)$ reducible must be flat. Thus, there is a good chance that all of Donaldson's polynomial invariants can be defined on $\overline{\mathscr{N}}_{d}(\omega)$ and thus on $\mathscr{M}_{d}(\omega)$. One major difficulty is that the classes in $H^{2}\left(\overline{\mathscr{N}}_{d}(\omega), \mathbb{Z}\right)$ associated to $H^{2}(X, \mathbb{Z}) \backslash H^{1,1}(X, \mathbb{Z})$ are defined as extensions of the first Chern classes of (nonholomorphic) smooth line bundles on $\mathscr{N}_{d}(\omega)$. Thus the obvious approach in algebraic geometry does not work. When $d$ is odd, there is a universal sheaf on $X \times \mathscr{M}_{d}(\omega)$. Tyurin [21] and O'Grady [19] introduced a series of polynomials $\left\{\tilde{\delta}_{k}\right\}$ by using the second Chern class of the universal sheaf. Their argument goes as follows. The second Chern class of the universal sheaf on $X \times \mathscr{M}_{d}(\omega)$ is a cohomology class in $H^{4}\left(X \times \mathscr{M}_{d}, \mathbb{Z}\right)$ and its component in $H^{2}(X, \mathbb{Z}) \otimes H^{2}\left(\mathscr{M}_{d}, \mathbb{Z}\right) \subset H^{4}\left(X \times \mathscr{M}_{d}, \mathbb{Z}\right)$ defines a homomorphism

$$
\rho_{\delta}: H_{2}(X, \mathbb{Z}) \rightarrow H^{2}\left(\mathscr{M}_{d}, \mathbb{Z}\right)
$$

Then for any $\alpha_{1}, \alpha_{2}, \cdots, \alpha_{k} \in H_{2}(X, \mathbb{Z})$ with $k=\operatorname{dim} \mathscr{M}_{d}$, define

$$
\tilde{\delta}\left(\alpha_{1}, \alpha_{2}, \cdots, \alpha_{k}\right)=\left[\rho_{\delta}\left(\alpha_{1}\right) \cup \rho_{\delta}\left(\alpha_{2}\right) \cup \cdots \cup \rho_{\delta}\left(\alpha_{k}\right)\right]\left(\mathscr{M}_{d}\right) \in \mathbb{Z} .
$$

Clearly the $\tilde{\delta}_{k}$ so defined are integer valued polynomials. By using the Grothendieck-Riemann-Roch theorem, one checks directly that the restriction of $(5.15)$ to $H^{1,1}(X, \mathbb{Z})$ coincides with (5.13), thus part of the $\tilde{\delta}_{k}$ are identical to $q_{k}$ according to Theorem 6 . The author conjectures that $\tilde{\delta}_{k}$ are identical to $w_{k}$ when $\omega$ is generic. (J. Morgan has given an affirmative answer to this question recently [16], [17].)

\section{Appendix}

Some of the material contained here is known to the experts. Due to a lack of references, we will present proofs of them. Let $U$ be an analytic open neighborhood of $0 \in \mathbb{C}^{\oplus 2}$ with coordinate chart $(x, y)$ and let $A$ be the quotient sheaf of $\mathscr{O}_{U}^{\oplus 2}$ supported on 0 . We denote by $F$ the coherent subsheaf of $\mathscr{O}_{U}^{\oplus 2}$ which is the kernel of $\mathscr{O}_{U}^{\oplus 2} \rightarrow A$.

Lemma 6.1. There are $f_{1}, \cdots, f_{n} \in \mathscr{O}_{U}^{\oplus 2}$ such that $f_{1}, \cdots, f_{n-2}$ are divisible by $x$ and that $f_{1}, \cdots, f_{n}$ generate the submodule $F \subset \mathscr{\sigma}_{U}^{\oplus 2}$.

Proof. Let $\bar{f}_{i}$ be the image of $f_{i}$ in $\mathscr{O}_{U}^{\oplus 2} /(x)$ under the homomorphism

$$
\rho: F \subset \mathscr{O}_{U}^{\oplus 2} \rightarrow \mathscr{O}_{U}^{\oplus 2} /(x)
$$


Without loss of generality, we can assume $\bar{f}_{n-1}$ and $\bar{f}_{n}$ generate the image $\rho(F)$. Then a linear combination of $f_{j}$ with $f_{n-1}$ and $f_{n}$ for $j=1, \cdots, n-2$ will provide a basis of $F$ as desired. q.e.d.

We can define a deformation of $F$ over the disk $D \in \mathbb{C}$ by using the basis constructed in Lemma 6.1. First let us rewrite $f_{j}(x, y)=x k_{j}(x, y)$ for $j=1, \cdots, n-2$ with $k_{j}(x, y) \in \mathscr{O}_{U}^{\oplus 2}$. Then define $F_{D}$ to be the subsheaf of $\mathscr{O}_{U \times D}^{\oplus 2}$ generated by the sections $(x-t) k_{1}(x, y), \cdots$, $(x-t) k_{n-2}(x, y), f_{n-1}(x, y), f_{n}(x, y)$. We denote them by $f_{i}(x, y, t)$ respectively. Let $A_{D}$ be the cokernel of the homomorphism $F_{D} \rightarrow \mathscr{O}_{U \times D}^{\oplus 2}$. Then we have the exact sequence

$$
0 \rightarrow F_{D} \rightarrow \mathscr{O}_{U \times D}^{\oplus 2} \rightarrow A_{D} \rightarrow 0 .
$$

In the sequel, we denote the sheaf $F_{D} \otimes k(t)$ (resp. $\left.A_{D} \otimes k(t)\right)$ by $F_{t}$ (resp. $A_{t}$ ) .

Lemma 6.2. There is a neighborhood $V \subset D$ of $0 \in D$ such that $A_{V}=A_{D \mid U \times V}$ is flat over $V$.

Proof. Let $t \in D$ be any closed point. By tensoring (6.1) with $k(t)$, we get the exact sequence

$$
0 \rightarrow \operatorname{Tor}\left(A_{D}, k(t)\right) \rightarrow F_{D} \otimes k(t) \rightarrow \mathscr{O}_{U}^{\oplus 2} \rightarrow A_{D} \otimes k(t) \rightarrow 0 .
$$

So to show that $A_{D}$ is flat at $t$, it suffices to show that $\operatorname{Tor}\left(A_{D}, k(t)\right)=0$ or, equivalently, $F_{D} \otimes k(t)$ is torsion free. We prove that $F_{D} \otimes k(0)$ is torsion free.

Assume $F_{D} \otimes k(0)$ is not torsion free. Then there exist $h \in F_{D}$ and $f \in \mathscr{O}_{U}$ such that $f \cdot h=t h^{\prime}$ for some $h^{\prime} \in F_{D}$. Let

$$
\begin{aligned}
h= & \sum_{i=1}^{n-2} g_{i}(x, y, t) \cdot(x-t) k_{i}(x, y) \\
& +g_{n-1}(x, y, t) \cdot f_{n-1}(x, y)+g_{n}(x, y, t) \cdot f_{n}(x, y) .
\end{aligned}
$$

Then the facts that $f(x, y) \cdot h \equiv 0, \bmod (t)$ in $\mathscr{O}_{U}^{\oplus 2}$ and $f_{n-1}(0, y)$, $f_{n}(0, y)$ generate the sheaf $\mathscr{O}_{U}^{\oplus 2} /(x)$ for $y \neq 0$ imply $x \mid g_{n-1}(x, y, 0)$, $x \mid g_{n}(x, y, 0)$, and

$$
\begin{aligned}
\sum_{i=1}^{n-2} g_{i}(x, y, 0) \cdot k_{i}(x, y) & +\frac{1}{x} g_{n-1}(x, y, 0) \cdot f_{n-1}(x, y) \\
& +\frac{1}{x} g_{n}(x, y, 0) \cdot f_{n}(x, y) \equiv 0 .
\end{aligned}
$$


Further, if we write $g_{i}(x, y, t)=\alpha_{i}(x, y)+t \beta_{i}(x, y, t)$, then the following identity holds in $\mathscr{O}_{U \times D}^{\oplus 2}$ :

$$
\begin{aligned}
h= & \sum_{i=1}^{n-2}\left[\left(\alpha_{i}(x, y) \cdot(x-t) k_{i}(x, y)+t \beta_{i}(x, y, t) \cdot(x-t) k_{i}(x, y)\right)\right] \\
& +\left[\left(\alpha_{n-1}(x, y) \cdot f_{n-1}(x, y)+t \beta_{n-1}(x, y, t) f_{n-1}(x, y)\right.\right. \\
& \left.\left.+\alpha_{n}(x, y) \cdot f_{n}(x, y)+t \beta_{n}(x, y, t) f_{n}(x, y)\right)\right] \\
= & (x-t)\left[\left(\sum_{i=1}^{n-2} \alpha_{i}(x, y) \cdot k_{i}(x, y)+\frac{1}{x} \alpha_{n-1}(x, y) B d f_{n-1}(x, y)\right.\right. \\
& \left.\left.+t\left[\left(\frac{1}{x} \alpha_{n-1}(x, y) \cdot f_{n-1}+\frac{1}{x} \alpha_{n}(x, y) \cdot f_{n}(x, y)\right)\right] f_{n}(x, y)\right)\right] \\
& +t \sum_{i=1}^{n} \beta_{i}(x, y, t) \cdot f_{i}(x, y, t) \\
= & t\left(\frac{1}{x} \alpha_{n-1}(x, y) \cdot f_{n-1}(x, y)+\frac{1}{x} \alpha_{n}(x, y) \cdot f_{n}(x, y)\right. \\
& \left.\left.\quad+\sum_{i=1}^{n} \beta_{i}(x, y, t) \cdot f_{i}(x, y, t)\right)\right]
\end{aligned}
$$

$=t h^{\prime \prime}$,

where $h^{\prime \prime}$ is an element of $F_{D} \subset \mathscr{O}_{U \times D}^{\oplus 2}$. Since $F_{D}$ is a submodule of $\mathscr{O}_{U \times D}^{\oplus 2}, h-t h^{\prime \prime}=0$ in $\mathscr{O}_{U \times D}^{\oplus 2}$ is equivalent to $h-t h^{\prime \prime}=0$ in $F_{D}$. Therefore, $h=0$ in $F_{D} \otimes k(0)$ or $F_{D} \otimes k(0)$ is torsion free.

Proposition 6.3. Assume that $A$ is a quotient sheaf of $\mathscr{O}_{U}^{\oplus 2}$ supported at $0 \in U$ with length $(A)=l \geq 2$. Then there is a flat family of $\mathscr{\sigma}_{U}^{\oplus 2}$ quotient sheaves $A_{t}, t \in D^{1} \subset \mathbb{C}$, such that $A_{0}=A$ and length $A_{t, x}<l$ for any $x \in U$.

Proof. Let $F_{0}$ be the kernel of $\mathscr{O}_{U}^{\oplus 2} \rightarrow A$, let $n=\operatorname{dim} F_{0} \otimes k(0)$, and let $f_{1}, \cdots, f_{n}$ be a basis of $F_{0}$. We first assume that for any $i=1, \cdots, n$, $f_{i}(0,0)=0$ and that the basis $f_{1}, \cdots, f_{n}$ has been rearranged according to Lemma 6.1. Note that then $n \geq 4$. Let $F_{t}$ be the flat family of torsion free sheaves constructed as in Lemma 6.2. We claim that except in some special cases, the cokernel $A_{t}$ of $F_{t} \rightarrow \mathscr{O}_{U}^{\oplus 2}$ gives the desired family. Indeed, for generic $t, F_{t}$ is generated by $(x-t) k_{1}(x, y), \cdots$, $(x-t) k_{n-2}(x, y), f_{n-1}(x, y), f_{n}(x, y)$. Let $y_{t}$ be the solution of 
$f_{n-1}(t, y) \wedge f_{n}(t, y)=0 \in \mathscr{O}_{U}$. Since $f_{n-1}(0,0) \wedge f_{n}(0,0)=0$ and $f_{n-1}(0, y) \wedge f_{n}(0, y) \neq 0$ for $y \neq 0$, such a solution $y_{t}$ does exist. Then $A_{t} \otimes k\left(\left(t, y_{t}\right)\right)$ is nontrivial. Assume $\operatorname{supp}\left(A_{t}\right)$ is a single point for generic $t$. Thus $k_{1}(x, y), \cdots, k_{n-2}(x, y)$ generates $\mathscr{O}_{U}^{\oplus 2}$ at the origin. So $n-2 \leq 2$ and then $n=4$. Further, $f_{1}$ and $f_{2}$ contain linear terms of $x$. Now by canceling the terms in $f_{3}$ and $f_{4}$ which involve $x$ by using $f_{1}$ and $f_{2}$, we see that $f_{2}$ and $f_{4}$ depend on $y$ only. If we exchange the variable $x$ and $y$ and let $\left(\bar{f}_{1}, \cdots, \bar{f}_{4}\right)=\left(f_{3}, f_{4}, f_{1}, f_{2}\right)$, then the family $\bar{F}_{t}$ provided in Lemma 6.2 will induce the right deformation unless $f_{3}$ and $f_{4}$ are linear in $y$ also. Therefore $A=\mathbb{C} \oplus \mathbb{C}$ and the deformation of it can be written down by hand. In case there is an $i$ such that $f_{i}(0,0) \neq 0$, then $F_{0}=\mathscr{O}_{U} \oplus \mathscr{I}$, where $\mathscr{I} \subset \mathscr{O}$ is the ideal sheaf of a zero scheme. The deformation can be found similarly. We leave it to the reader.

Proposition 6.4. Let $A$ be any quotient sheaf of $\mathscr{O}_{U}^{\oplus 2}$ supported on the origin $0 \in U$. Then there is a deformation $A_{t}$ of $A$ as quotient sheaves of $\mathscr{O}_{U}^{\oplus 2}$ such that for generic $t, A_{t}$ is supported at $l$ distinct points, where $l=$ length $A_{0}$.

Proof. By Proposition 6.3, for any quotient sheaf $A$, there is a deformation $A_{t}$ such that for generic $t$ and any $p \in U$, length $\left(A_{t}\right)_{p}<$ length $(A)_{0}$. We use the obvious fact that a deformation of a deformation is still a deformation. Then by applying Proposition 6.3 successively, we conclude that there is a deformation $A_{t}$ of $A$ so that for generic $t$, length $A_{t, x} \leq 1$ for any $x \in U$. q.e.d.

The following proposition is of its own interest. To the author's knowledge, the question whether Quot $_{E}^{c}(l)$ is irreducible is still open.

Proposition 6.5 (Lemma 3.4). Let $E$ be any locally free polystable sheaf and let $l: X \rightarrow \mathbb{Z}^{+}$be any integer function. Assume $c_{2}(E)+\sum_{x} l(x) \leq d$. Then $\mathscr{Q}_{l}^{\mu}(E)$ is a closed, connected algebraic subset of $\mathscr{Q}^{\mu}(E)$.

Proof. Since we do not need the closedness in this paper, we leave the proof to the reader.

Now we prove the connectedness of the set $\mathscr{Q}_{l}^{\mu}(E)$. Let Quot ${ }_{E}^{c}$ be the Grothendieck quotient scheme parametrizing all quotient sheaves $A$ of $E$ with length $(A)=c$. For the given function $l$, let

$$
\operatorname{Quot}_{E}^{c}(l)=\left\{A \in \operatorname{Quot}_{E}^{c} \mid \text { length }(A)_{x} \geq l(x) \text { for any } x \in X\right\} .
$$

It is easy to see that $\mathscr{Q}_{l}^{\mu}(E)$ is connected if and only if $\operatorname{Quot}_{E}^{c}(l)$ is connected.

We continue our proof by working in the analytic category. Assume $x_{1}, x_{2}, \cdots, x_{k} \in X$ are the points where $l(x) \neq 0$. For $i=1, \cdots, k$, 
let $\left\{U_{i}^{n}\right\}_{n=1}^{\infty}$ be sequences of decreasing connected open subsets of $X$ such that the closure $\bar{U}_{i}^{n}$ satisfies $\bigcap_{n=1}^{\infty} \bar{U}_{i}^{n}=\left\{x_{i}\right\}$. Then define

$$
\operatorname{Quot}_{E}^{c}(l)_{n}=\left\{A \in \operatorname{Quot}_{E}^{c} \mid \sum_{x \in U_{i}^{n}} \operatorname{length}(A)_{x} \geq l\left(x_{i}\right), 1 \leq i \leq k\right\} \text {. }
$$

Quot $_{E}^{c}(l)_{n}$ is a decreasing sequence of open subsets of Quot ${ }_{E}^{c}$. We claim that $\operatorname{Quot}_{E}^{c}(l)_{n}$ is connected. Indeed, thanks to Proposition 6.4, any $A \in$ Quot $_{E}^{c}(l)_{n}$ can be deformed within Quot ${ }_{E}^{c}(l)_{n}$ to a quotient sheaf $A^{\prime}$ such that length $A_{x}^{\prime} \leq 1$ for all $x \in X$. Clearly, all such $A^{\prime}$ form a connected set. So $\operatorname{Quot}_{E}^{c}(l)_{n}$ is connected. Note that

$$
\operatorname{Quot}_{E}^{c}(l)=\bigcap_{n=1}^{\infty}\left(\operatorname{Quot}_{E}^{c}(l)_{n}\right)^{\text {closure }}
$$

and the right-hand side is a decreasing sequence of closed, connected subsets of the compact ambient space $\operatorname{Quot}_{E}^{c}$. Therefore, Quot ${ }_{E}^{c}(l)$ is connected.

\section{Acknowledgment}

The author acknowledges that the current work benefitted from talks with S. Y. Cheng, D. Gieseker, R. Lazarsfeld, C. Rippel, C. Taubes, and S. T. Yau. The author thanks D. Gieseker especially for his kindness and patience in explaining various concepts in algebraic geometry and for his time and effort spent in reviewing the manuscript. His suggestions have made the paper more readable. The author would also like to express his gratitude for the hospitality of the Department of Mathematics at UCLA. This work is partially supported by an NSF grant.

\section{Bibliography}

[1] S. K. Donaldson, Anti-self-dual Yang-Mills connections over complex algebraic surfaces and stable vector bundles, Proc. London Math. Soc. (3) 50 (1985) 1-26.

[2] __ Connections, cohomology and the intersection forms of 4-manifolds, J. Differential Geometry 24 (1986) 275-341.

[3] __ Polynomial invariants for smooth four-manifolds, Topology 29 (1990) 257-315.

[4] J.-M. Drezet \& M. S. Narasimhan, Group de Picard des varietes de modules de fibres semi-stables sur les coubes algebriques, Invent. Math. 97 (1989) 53-94.

[5] D. Freedman \& K. Uhlenbeck, Instantons and four-manifolds, Springer, Berlin, 1984.

[6] R. Friedman, Vector bundles over surfaces, to appear.

[7] R. Friedman \& J. W. Morgan, Smooth four-manifolds and complex surfaces, preprint.

[8] D. Gieseker, On the moduli of vector bundles on an algebraic surface, Ann. of Math. (2) 106 (1977) 45-60. 
[9] A. Grothendieck, Techniques de construction et théorèmes d'existence en géométrie algébrique IV: les schémas de Hilbert, Séminaire Bourbaki, 1960-61.

[10] R. Hartshorne, Algebraic geometry, Graduate Texts in Math., Vol. 52, Springer, Berlin, 1977.

[11] M. Itoh, The moduli space of Yang-Mills connections over a Kähler surface is a complex manifold, Osaka J. Math. 22 (1985) 845-862.

[12] F. Knudsen \& D. Mumford, The projectivity of the moduli space of stable curves I: preliminaries on 'det' and 'div', Math. Scand. 39 (1976) 19-55.

[13] J. Li, Kodaira dimension of moduli space of vector bundle on algebraic surfaces, preprint.

[14] M. Maruyama, Moduli of stable sheaves, I, J. Math. Kyoto Univ. 17 (1977) 91-126.

[15] __ Moduli of stable sheaves. II, J. Math. Kyoto Univ. 18 (1978) 557-614.

[16] J. W. Morgan, Comparison of the Gieseker compactification and the Uhlenbeck compactification of moduli space, preprint.

[17] _ Comparison of the Donaldson polynomial invariants with their algebro-geometric analogue, preprint.

[18] D. Mumford, Geometri invariant theory, Springer, Berlin, 1982.

[19] K. G. O'Grady, Algebro-geometric analogues of Donaldson's polynomials, preprint.

[20] C. Taubes, Self-dual connections on 4-manifolds with indefinite intersection matrix, J. Differential Geometry 19 (1984) 517-560.

[21] A. N. Tyurin, Algebraic geometric aspects of smooth structures I. The Donaldson polynomials, Russian Math. Surveys 44 (1990) 113-178.

[22] K. Uhlenbeck, Removable singularity in Yang-Mills fields, Comm. Math. Phys. 83 (1982) 11-29.

UNiversity of CALIFornia, Los ANGeles 